A Study of Detonation Diffraction in the Ignition-and-Growth Model

A. K. Kapila, D. W. Schwendeman, J. B. Bdzil, W. D. Henshaw

April 20, 2006

Combustion Theory and Modelling 
This document was prepared as an account of work sponsored by an agency of the United States Government. Neither the United States Government nor the University of California nor any of their employees, makes any warranty, express or implied, or assumes any legal liability or responsibility for the accuracy, completeness, or usefulness of any information, apparatus, product, or process disclosed, or represents that its use would not infringe privately owned rights. Reference herein to any specific commercial product, process, or service by trade name, trademark, manufacturer, or otherwise, does not necessarily constitute or imply its endorsement, recommendation, or favoring by the United States Government or the University of California. The views and opinions of authors expressed herein do not necessarily state or reflect those of the United States Government or the University of California, and shall not be used for advertising or product endorsement purposes. 


\title{
A Study of Detonation Diffraction in the Ignition-and-Growth Model
}

\author{
A. K. Kapila and D. W. Schwendeman \\ Department of Mathematical Sciences, Rensselaer Polytechnic Institute, Troy, NY \\ J. B. Bdzil \\ DX-2 Group, Los Alamos National Laboratory, Los Alamos, NM \\ W. D. Henshaw \\ CASC, Lawrence Livermore National Laboratory, Livermore, CA
}

March 28, 2006

\begin{abstract}
Heterogeneous high-energy explosives are morphologically, mechanically and chemically complex. As such, their ab-initio modeling, in which well-characterized phenomena at the scale of the microstructure lead to a rationally homogenized description at the scale of observation, is a subject of active research but not yet a reality. An alternative approach is to construct phenomenological models, in which forms of constitutive behavior are postulated with an eye on the perceived picture of the micro-scale phenomena, and which are strongly linked to experimental calibration. Most prominent among these is the ignition-and-growth model conceived by Lee and Tarver. The model treats the explosive as a homogeneous mixture of two distinct constituents, the unreacted explosive and the products of reaction. To each constituent is assigned an equation of state, and a single reaction-rate law is prescribed for the conversion of the explosive to products. It is assumed that the two constituents are always in pressure and temperature equilibrium.

The purpose of this paper is to investigate in detail the behavior of the model in situations where a detonation turns a corner and undergoes diffraction. A set of parameters appropriate for the explosive LX17 is selected. The model is first examined analytically for steady, planar, 1-D solutions and the reaction-zone structure of Chapman-Jouguet detonations is determined. A computational study of two classes of problems is then undertaken. The first class corresponds to planar, 1-D initiation by an impact, and the second to corner turning and diffraction in planar and axisymmetric geometries. The 1-D initiation, although interesting in its own right, is utilized here as a means for interpretation of the 2-D results. It is found that there are two generic ways in which 1-D detonations are initiated in the model, and that these scenarios play a part in the post-diffraction evolution as well. For the parameter set under study the model shows detonation failure, but only locally and temporarily, and does not generate sustained dead zones. The computations employ adaptive mesh refinement and are finely resolved. Results are obtained for a rigid confinement of the explosive. Compliant confinement represents its own computational challenges and is currently under study. Also under development is an extended ignition-and-growth model which takes into account observed desensitization of heterogeneous explosives by weak shocks.
\end{abstract}

\section{Introduction}

Mathematical modeling of heterogeneous high-energy explosives poses a vexing problem. These materials have a complex microstructure in which crystalline fragments of the energetic material are held together by a plastic binder, and voids and pores abound within the granular aggregate. It is observed that when a sample of such an explosive is subjected to a sufficiently strong stimulus, such as impact by a high-velocity 
projectile, a detonation is initiated. Our knowledge of the thermo-mechanical behavior of the material over the broad ranges of pressure and temperature encountered in a detonation is incomplete, and the same is true of the complex set of reactions that are responsible for the liberation of energy. The morphological complexity, the dearth of information, and the multi-scale nature of material response are insurmountable blocks in the way of any attempt at ab-initio modeling of the detonation phenomena, at least at the present time.

On certain aspects of the problem, however, there is broad agreement. It is known that while the crystalline explosive possesses a strong ignition threshold, relatively weaker stimuli are sufficient to initiate the heterogeneous explosive. This propensity is attributed to the creation of a nonuniform temperature distribution when the heterogeneous aggregate is exposed to the initiating shock. Discrete sites, where the local temperature far exceeds the bulk average, are generated as a result of mechanical processes such as friction, pore collapse, shear banding and local plastic deformations. These sites, or hot spots, act as preferred locations of ignition, where burning commences and then spreads to consume the entire bulk. With this picture in mind, efforts have been directed at constructing phenomenological, macro-scale, continuum-type models. These include the Forest-fire model [1, 2], the JTF model [3] and the HVRB model [4], but the most celebrated of the lot is the ignition-and-growth model, originally derived by Lee and Tarver [5] and later refined and utilized by Tarver and colleagues $[6,7,8,9,10]$. Additional references can be found in the recent paper by Tarver [11].

The model treats the explosive as a homogeneous mixture of two distinct constituents: (i) the unreacted explosive and (ii) the products of reaction. To each constituent is assigned an equation of state, and a single reaction-rate law is postulated for the conversion of the explosive to products. It is assumed that the two constituents are always in pressure and temperature equilibrium, and that the energy and volume of the mixture is the sum of the corresponding quantities for the individual constituents, weighted by the variable that measures the progress of reaction. The model contains a large number of parameters which are experimentally calibrated to the explosive of interest.

The model has had considerable success, though less as a tool which, once calibrated to a certain suite of experiments, has the ability to predict behavior broadly, such as in an entirely different set of experiments. Rather, its success lies in it providing a framework within which different classes of experiments can be simulated and studied. While the framework holds firm, each new class of experiments may, and indeed does, require tuning of the parameters within the general framework. For example, experiments in which the initiation process is the focus of interest require a significantly different parameter set than experiments that concern propagation of established detonations $[7,11]$. The phenomenology exhibited by the model depends upon the resolution of the computations as well, and it appears that some of the simulations reported in the literature are not adequately resolved $[12,13]$.

Recently the model has been applied to study diffraction of detonations as they turn sharp corners [11, 13]. Experiments suggest that dead zones, or sustained pockets of unreacted material, may appear in the vicinity of the corners [13]. There appears to be some disagreement in the literature as to exactly what the model predicts in these situations; studies on similar configurations reach opposite conclusions. For example, in the so-called hockey-puck geometry, Souers et al [13] report no sustained dead zones while Tarver [11] suggests that the model does capture failure.

The purpose of this paper is to examine in detail the solution set of the model, in the context of diffraction, to find out exactly what phenomena are contained within the model. We believe that once a model has been constructed for any physical situation, it is essential that it be thoroughly analyzed, and its properties exhaustively investigated, so that its strengths and weaknesses are fully understood. Here we proceed by selecting a single set of parameters for the explosive LX-17, this set having been reported as being more appropriate for detonation propagation rather than detonation initiation [11]. We examine it analytically for steady, planar, 1-D solutions and determine the reaction-zone structure of Chapman-Jouguet detonations. We then carry out a computational study of two classes of problems. The first class corresponds to planar, 1-D initiation by an impact, and the second to corner turning and diffraction in planar and axisymmetric configurations. The 1-D initiation is interesting in its own right, and one can argue that it should properly be studied by using the parameter set prescribed for it [11]. However, our purpose here is to employ the 1-D initiation results as a means by which the 2-D results can be profitably interpreted. We find that there are two generic ways in which 1-D detonations are initiated according to the model, and that these 
scenarios play a part in the post-diffraction evolution as well. We find, in particular, that for the parameter set under study the model does show detonation failure, but only locally and temporarily, and that it does not generate sustained dead zones. Our computations employ adaptive mesh refinement following the work by Henshaw and Schwendeman [14], and are finely resolved. To our knowledge diffraction computations for condensed explosives at such high resolution have not appeared in the literature. In the gaseous phase, however, high-resolution diffraction computations do exist; see, in particular, Arienti and Shepherd [15]. Our results are obtained for a rigid confinement of the explosive. Compliant confinement, representing its own computational challenges, will alter the results to some extent and is currently under study. Also under development is an extended ignition-and-growth model which takes into account observed desensitization of heterogeneous explosives by weak shocks.

The framework of the model is presented in section 2. Steady traveling waves are investigated in section 3 , including shock conditions, Hugoniot curves and the Chapman-Jouguet state. The equation set is rendered dimensionless in section 4. Section 5 displays the LX-17 data set, and the corresponding Hugoniots and reaction-zone profiles for the CJ detonations. Section 6 outlines the numerical method. The 1-D shockinitiation problem is investigated in section 7 , and the planar and axisymmetric diffraction problems in sections 8-10. The paper ends with conclusions drawn in section 11.

\section{The Model}

\subsection{Equations of State}

The ignition-and-growth model treats the heterogeneous explosive as a homogeneous mixture of two constituents: (i) the unreacted explosive and (ii) the reaction products. The consequences of microstructural heterogeneity, not reflected in the thermo-mechanical description, are accounted for in an impressionistic way, as we shall see, in the formulation of the reaction rate. Separate JWL equations of state [16] are prescribed for each constituent; the equation of state for the reactant is fitted to the available shock Hugoniot data and the product equation of state to data from cylinder test and other metal acceleration experiments [11]. These equations have the following mechanical and thermal forms.

Mechanical:

$$
\begin{aligned}
& \tilde{E}_{s}=\frac{\tilde{p}_{s} v_{s}}{\omega_{s}}-\tilde{F}_{s}\left(v_{s}\right)+\tilde{F}_{s}(1), \\
& \tilde{E}_{g}=\frac{\tilde{p}_{g} v_{g}}{\omega_{g}}-\tilde{F}_{g}\left(v_{g}\right)-\tilde{Q} .
\end{aligned}
$$

Thermal:

$$
\begin{aligned}
& \tilde{p}_{s}=\frac{\omega_{s}}{v_{s}}\left[\tilde{C}_{s} \tilde{T}_{s}+\tilde{G}_{s}\left(v_{s}\right)+\tilde{F}_{s}\left(v_{s}\right)\right] \\
& \tilde{p}_{g}=\frac{\omega_{g}}{v_{g}}\left[\tilde{C}_{g} \tilde{T}_{g}+\tilde{G}_{g}\left(v_{g}\right)+\tilde{F}_{g}\left(v_{g}\right)\right] .
\end{aligned}
$$

Here, the subscript $s$ refers to the unreacted solid explosive and $g$ to the gaseous products of reaction, $\tilde{p}_{i}$ is the pressure and $\tilde{T}_{i}$ the temperature of constituent $i$. The quantity $v_{i}$, appearing in the arguments of the functions $\tilde{F}_{i}$ and $\tilde{G}_{i}$, is the specific volume of constituent $i$ scaled by a reference volume $\tilde{v}_{s 0}$, i.e., $v_{i}=\tilde{v}_{i} / \tilde{v}_{s 0}$. We choose $\tilde{v}_{s 0}$ to be the specific volume of the solid reactant in the ambient state. In the equations of state the energies $\tilde{E}_{i}$ are in units of energy per unit volume of the unreacted solid [17], and are related to the specific energies per unit mass, $\tilde{e}_{i}$, by

$$
\tilde{e}_{i}=\tilde{v}_{s 0} \tilde{E}_{i}, \quad i=s \text { or } g .
$$

The energy of detonation is denoted by $\tilde{Q}$, and the constant $\tilde{F}_{s}(1)$ has been added to the right-hand side 
of (1) to ensure that $\tilde{E}_{s}=0$ at the upstream state. The functions $\tilde{F}_{i}$ and $\tilde{G}_{i}$ are given by

$$
\begin{aligned}
& \tilde{F}_{s}(V)=\tilde{A}_{s}\left(\frac{V}{\omega_{s}}-\frac{1}{R_{1_{s}}}\right) \exp \left(-R_{1_{s}} V\right)+\tilde{B}_{s}\left(\frac{V}{\omega_{s}}-\frac{1}{R_{2_{s}}}\right) \exp \left(-R_{2_{s}} V\right), \\
& \tilde{F}_{g}(V)=\tilde{A}_{g}\left(\frac{V}{\omega_{g}}-\frac{1}{R_{1_{g}}}\right) \exp \left(-R_{1_{g}} V\right)+\tilde{B}_{g}\left(\frac{V}{\omega_{g}}-\frac{1}{R_{2_{g}}}\right) \exp \left(-R_{2_{g}} V\right),
\end{aligned}
$$

and

$$
\begin{aligned}
& \tilde{G}_{s}(V)=\frac{\tilde{A}_{s}}{R_{1_{s}}} \exp \left(-R_{1_{s}} V\right)+\frac{\tilde{B}_{s}}{R_{2_{s}}} \exp \left(-R_{2_{s}} V\right), \\
& \tilde{G}_{g}(V)=\frac{\tilde{A}_{g}}{R_{1_{g}}} \exp \left(-R_{1_{g}} V\right)+\frac{\tilde{B}_{g}}{R_{2_{g}}} \exp \left(-R_{2_{g}} V\right) .
\end{aligned}
$$

The constants $\tilde{A}_{i}, \tilde{B}_{i}, \omega_{i}, \tilde{C}_{i}, R_{1_{i}}$ and $R_{2_{i}}, i=s$ or $g$, characterize the explosive under study.

We observe that the equation of state for each constituent is of Mie-Gruneisen form with constant Gruneisen gamma $\omega_{i}$ and constant heat capacity $\tilde{C}_{i}$. Each EOS also satisfies the thermodynamic constraint

$$
\left[\left(\frac{\partial \tilde{e}_{i}}{\partial \tilde{v}_{i}}\right)_{\tilde{p}_{i}}+\tilde{p}_{i}\right]\left(\frac{\partial \tilde{T}_{i}}{\partial \tilde{p}_{i}}\right)_{\tilde{v}_{i}}-\left(\frac{\partial \tilde{e}_{i}}{\partial \tilde{p}_{i}}\right)_{\tilde{v}_{i}}\left(\frac{\partial \tilde{T}_{i}}{\partial \tilde{v}_{i}}\right)_{\tilde{p}_{i}}=\tilde{T}_{i} .
$$

(See Fickett and Davis [16], Appendix 4B.)

\subsection{The Reaction Rate}

The chemical reaction rate equation in the ignition-and-growth model is a pressure-driven rule, of the form

$$
\begin{aligned}
\frac{d \lambda}{d t}=\tilde{R}=\tilde{I} & (1-\lambda)^{b}(1 / v-1-a)^{x} H\left(\lambda_{\mathrm{ig}, \max }-\lambda\right) \\
& +\tilde{G}_{1}(1-\lambda)^{c} \lambda^{d} \tilde{p}^{y} H\left(\lambda_{G_{1}, \max }-\lambda\right)+\tilde{G}_{2}(1-\lambda)^{e} \lambda^{g} \tilde{p}^{z} H\left(\lambda-\lambda_{G_{2}, \min }\right) .
\end{aligned}
$$

Here $\lambda$ is the reaction progress variable (i.e., the mass fraction of the product), $H$ is the Heaviside function, and $\tilde{I}, \tilde{G}_{1}, \tilde{G}_{2}, a, b, c, d, e, g, x, y$ and $z$ are constants. Three switching constants, $\lambda_{\mathrm{ig}, \max }$, $\lambda_{G_{1}, \max }$ and $\lambda_{G_{2} \text { min }}$ also appear, and limit the contributions of the three terms to respectively a maximum reacted fraction $\lambda_{\mathrm{ig}, \max }$ for the first term, a maximum reacted fraction $\lambda_{G_{1}, \max }$ for the second term, and a minimum reacted fraction $\lambda_{G_{2} \text {, min }}$ for the third term. The constant $a$ in the first term defines a compression threshold that must be exceeded before ignition will occur. The exponents $b, c, d, e$ and $g$ are topological constants intended to mimic the transition from a hole-burning to a grain-burning configuration as $\lambda$ varies from 0 to 1 . The pressure exponents $y$ and $z$ are measures of the rate sensitivity of the reaction rate. The three terms are designed to model the three stages of reaction that are believed to occur in shock initiation and propagation of detonations in heterogeneous explosives. The first term represents ignition of the explosive by hot spots, generated by the passage of the initiating shock over material inhomogeneities. The remaining two terms have different interpretations, depending upon whether shock initiation or detonation propagation is being modeled. For shock initiation the second term represents the growth of reaction as deflagrations originating at the hot spots spread into the surrounding bulk, either as holes burning outwards or grains burning inwards. The third term describes the swift transition to detonation as the growing hot spots begin to coalesce and transfer large amounts of energy to the unreacted explosive, causing it to react rapidly. For detonation propagation the second reaction models the rapid formation of major gaseous products of reaction, while the third term describes the relatively slow diffusion-controlled formation of solid carbon. Accordingly, the parameter sets for the two situations are quite different. It is also worth pointing out that calibrations of the equation of state and rate function are coupled; any change in the calibration of the equation of state requires a corresponding change in the rate. 


\subsection{The Mixture Rules}

The following mixture rules define the specific energy $\tilde{e}$ and specific volume $\tilde{v}$ of the mixture in terms of the corresponding quantities for the constituents and the reaction progress variable $\lambda$ :

$$
\begin{aligned}
& \left.\tilde{e}=(1-\lambda) \tilde{e}_{s}\left(\tilde{p}_{s}, \tilde{v}_{s}\right)+\lambda \tilde{e}_{g}\left(\tilde{p}_{g}, \tilde{v}_{g}\right)=\tilde{v}_{s_{0}}\left[(1-\lambda) \tilde{E}_{s}+\lambda \tilde{E}_{g}\right)\right], \\
& \tilde{v}=(1-\lambda) \tilde{v}_{s}+\lambda \tilde{v}_{g} .
\end{aligned}
$$

It is assumed that both constituents move with the same speed $\tilde{u}$, and further that they are in pressure and temperature equilibrium, i.e.,

$$
\tilde{p}_{s}=\tilde{p}_{g}=\tilde{p}, \quad \tilde{T}_{s}=\tilde{T}_{g}=\tilde{T} .
$$

While pressure equilibrium is easier to justify at the high pressures involved in detonations, temperature equilibrium is less so [12].

In equation (11), $\tilde{E}_{s}$ and $\tilde{E}_{g}$ can be replaced by the corresponding expressions from (1) and (2), keeping in mind that pressure equilibrium requires $\tilde{p}_{s}=\tilde{p}_{g}=\tilde{p}$. The result is

$$
\tilde{e}=\tilde{v}_{s 0}(1-\lambda)\left\{\frac{\tilde{p} v_{s}}{\omega_{s}}-\tilde{F}_{s}\left(v_{s}\right)+\tilde{F}_{s}(1)\right\}+\tilde{v}_{s_{0}} \lambda\left\{\frac{\tilde{p} v_{g}}{\omega_{g}}-\tilde{F}_{g}\left(v_{g}\right)-\tilde{Q}\right\} .
$$

Similarly, upon equating $\tilde{T}_{s}$ and $\tilde{T}_{g}$ from (3) and (4), and enforcing pressure equilibrium, we get

$$
\tilde{p}\left[\frac{v_{s}}{\omega_{s} \tilde{C}_{s}}-\frac{v_{g}}{\omega_{g} \tilde{C}_{g}}\right]-\frac{1}{\tilde{C}_{s}}\left[\tilde{F}_{s}\left(v_{s}\right)+\tilde{G}_{s}\left(v_{s}\right)\right]+\frac{1}{\tilde{C}_{g}}\left[\tilde{F}_{g}\left(v_{g}\right)+\tilde{G}_{g}\left(v_{g}\right)\right]=0 .
$$

We can also write the mixture volume equation (12) in terms of the scaled volumes as

$$
(1-\lambda) v_{s}+\lambda v_{g}=v .
$$

Equations (15) and (16) are, in principle, the relations that yield $v_{s}$ and $v_{g}$ (or equivalently, $\tilde{v}_{s}$ and $\tilde{v}_{g}$ ) in terms of $\lambda, \tilde{p}$ and $v$ (or $\tilde{v}$ ). Then, equation (14) is effectively the (implicit) expression for the mixture energy $\tilde{e}$ in terms of $\lambda$ and the mixture variables $\tilde{p}$ and $\tilde{v}$.

\subsection{The Balance Laws}

It is assumed that the mixture variables satisfy the reactive Euler equations. In a two-dimensional geometry these equations are

$$
\tilde{\mathbf{u}}_{\tilde{t}}+\tilde{\mathbf{f}}(\tilde{\mathbf{u}})_{\tilde{x}}+\tilde{\mathbf{g}}(\tilde{\mathbf{u}})_{\tilde{y}}=\tilde{\mathbf{h}}(\tilde{\mathbf{u}}),
$$

where the vector of state variables $\tilde{\mathbf{u}}$, the flux vectors $\tilde{\mathbf{f}}$ and $\tilde{\mathbf{g}}$, and the source term $\tilde{\mathbf{h}}$ are given by

$$
\tilde{\mathbf{u}}=\left[\begin{array}{c}
\tilde{\rho} \\
\tilde{\rho} \tilde{u} \\
\tilde{\rho} \tilde{w} \\
\tilde{\rho} \tilde{\mathcal{E}} \\
\tilde{\rho} \lambda
\end{array}\right], \quad \tilde{\mathbf{f}}=\left[\begin{array}{c}
\tilde{\rho} \tilde{u} \\
\tilde{\rho} \tilde{u}^{2}+\tilde{p} \\
\tilde{\rho} \tilde{u} \tilde{w} \\
\tilde{u}(\tilde{\rho} \tilde{\mathcal{E}}+\tilde{p}) \\
\tilde{\rho} \tilde{u} \lambda
\end{array}\right], \quad \tilde{\mathbf{g}}=\left[\begin{array}{c}
\tilde{\rho} \tilde{w} \\
\tilde{\rho} \tilde{w} \tilde{u} \\
\tilde{\rho} \tilde{w}^{2}+\tilde{p} \\
\tilde{w}(\tilde{\rho} \tilde{\mathcal{E}}+\tilde{p}) \\
\tilde{\rho} \tilde{w} \lambda
\end{array}\right], \quad \tilde{\mathbf{h}}=\left[\begin{array}{c}
0 \\
0 \\
0 \\
0 \\
\tilde{\rho} \tilde{R}
\end{array}\right]
$$

Here $\tilde{\rho}=1 / \tilde{v}$ is the density, $(\tilde{u}, \tilde{w})$ the $(\tilde{x}, \tilde{y})$ components of velocity, $\tilde{R}$ the reaction rate, and $\tilde{\mathcal{E}}$ the total energy per unit mass, given by

$$
\tilde{\mathcal{E}}=\tilde{e}+\frac{1}{2}\left(\tilde{u}^{2}+\tilde{w}^{2}\right)
$$

We have already specified the ambient specific volume of the reactant. The complete ambient state is

$$
\lambda_{s_{0}}=0, \quad p_{s_{0}}=0, \quad \text { and } \quad \tilde{v}_{s_{0}} .
$$




\section{$3 \quad$ Steady, Traveling Waves}

We begin our analysis of the model by considering a steady, planar wave traveling with speed $\tilde{D}_{0}$ into the ambient, unreacted explosive. When considered in a wave-fixed frame of reference, the conservation equations for mass, $\tilde{x}$-momentum and energy in equation set (18) yield

$$
\begin{aligned}
\frac{\tilde{U}}{\tilde{v}} & =\frac{\tilde{D}_{0}}{\tilde{v}_{s_{0}}}, \\
\tilde{p}+\frac{\tilde{U}^{2}}{\tilde{v}} & =\frac{\tilde{D}_{0}^{2}}{\tilde{v}_{s_{0}}}, \\
\tilde{e}+\tilde{p} \tilde{v}+\frac{1}{2} \tilde{U}^{2} & =\tilde{e}_{0}+\frac{1}{2} \tilde{D}_{0}^{2} .
\end{aligned}
$$

Here, $\tilde{U}=\tilde{D}_{0}-\tilde{u}$ is the particle velocity in the wave-fixed frame, and the suffix 0 refers to the state of the unreacted solid far upstream given in (19). From (20) and (21) we obtain the relation

$$
\tilde{U}=\tilde{D}_{0} v
$$

and the Rayleigh line

$$
\tilde{p}=\frac{\tilde{D}_{0}^{2}}{\tilde{v}_{s_{0}}}(1-v)
$$

\subsection{Hugoniot Curves}

It is instructive to consider the Hugoniot curves, parametrized by reaction progress $\lambda$, in the $\tilde{p} \tilde{v}$-plane. To generate a $\lambda$-Hugoniot, we fix $\lambda$ and start with the energy balance (22). There, we use (23) to substitute for $\tilde{U}$ and (14) to substitute for $\tilde{e}$. A division by $\tilde{v}_{s_{0}}$ then leads to

$$
(1-\lambda)\left\{\frac{\tilde{p} v_{s}}{\omega_{s}}-\tilde{F}_{s}\left(v_{s}\right)+\tilde{F}_{s}(1)\right\}+\lambda\left\{\frac{\tilde{p} v_{g}}{\omega_{g}}-\tilde{F}_{g}\left(v_{g}\right)-\tilde{Q}\right\}+\tilde{p} v+\frac{1}{2} \frac{\tilde{D}_{0}^{2}}{\tilde{v}_{s_{0}}}\left(v^{2}-1\right)=0,
$$

where $\tilde{e}_{0}=0$. Elimination of $\tilde{D}_{0}$ with the help of the Rayleigh-line expression (24), followed by some rearrangement, gives

$$
\tilde{p}\left\{\frac{(1-\lambda) v_{s}}{\omega_{s}}+\frac{\lambda v_{g}}{\omega_{g}}+\frac{v-1}{2}\right\}+(1-\lambda)\left\{F_{s}(1)-F_{s}\left(v_{s}\right)\right\}-\lambda F_{g}\left(v_{g}\right)=\lambda \tilde{Q} .
$$

Elimination of $v_{s}$ and $v_{g}$ with the help of (15) and (16) then yields the Hugoniot implicitly. The special cases corresponding to $\lambda=0$ and $\lambda=1$ can be obtained explicitly, and are given below.

$\underline{\lambda=0}$

$$
\tilde{p}\left\{\left(\frac{2+\omega_{s}}{2 \omega_{s}}\right) v-\frac{1}{2}\right\}+\tilde{F}_{s}(1)-\tilde{F}_{s}(v)=0,
$$

$\underline{\lambda=1}$

$$
\tilde{p}\left\{\left(\frac{2+\omega_{g}}{2 \omega_{g}}\right) v-\frac{1}{2}\right\}-\tilde{F}_{g}(v)=\tilde{Q}
$$

We observe that unlike the case of an ideal gas, the two extreme Hugoniots have different vertical asymptotes. In section 5, we provide graphs of the Hugoniots for a particular explosive.

\subsection{The State Behind the Lead Shock}

For a detonation traveling with speed $\tilde{D}_{0}$ into the unreacted material, the state behind the shock is determined by eliminating $\tilde{p}$ between the Rayleigh line, (24) and the $\lambda=0$ Hugoniot, (26). The result is

$$
\frac{\tilde{D}_{0}^{2}}{\tilde{v}_{s_{0}}}(1-v)\left\{\left(\frac{2+\omega_{s}}{2 \omega_{s}}\right) v-\frac{1}{2}\right\}+\tilde{F}_{s}(1)-\tilde{F}_{s}(v)=0,
$$


or, after some rearrangement,

$$
v^{2}-2\left(\frac{1+\omega_{s}}{2+\omega_{s}}\right) v+\frac{\omega_{s}}{2+\omega_{s}}-\left(\frac{2 \omega_{s}}{2+\omega_{s}}\right) \frac{\tilde{v}_{s_{0}}}{\tilde{D}_{0}^{2}}\left[\tilde{F}_{s}(1)-\tilde{F}_{s}(v)\right]=0 .
$$

Presumably this equation has two roots; one is clearly $v=1$ which corresponds to no shock. The second root, $v^{\mathrm{S}}$ say, gives the value of $v$ behind the shock as a function of the shock speed $\tilde{D}_{0}$. Then, (23) gives the particle speed and (24) the pressure behind the shock as

$$
\tilde{U}^{\mathrm{S}}=\tilde{D}_{0} v^{\mathrm{S}}, \quad \tilde{p}^{\mathrm{S}}=\frac{\tilde{D}_{0}^{2}}{\tilde{v}_{s_{0}}}\left(1-v^{\mathrm{S}}\right) .
$$

\subsection{The Chapman-Jouguet State}

For detonation speed $\tilde{D}_{0}$. the state at the end of the reaction zone is found by eliminating $\tilde{p}$ between the fully reacted Hugoniot (27) and the Rayleigh line (24). The result is

$$
v^{2}-2\left(\frac{1+\omega_{g}}{2+\omega_{g}}\right) v+\frac{\omega_{g}}{2+\omega_{g}}+\left(\frac{2 \omega_{g}}{2+\omega_{g}}\right) \frac{\tilde{v}_{s_{0}}}{\tilde{D}_{0}^{2}}\left[\tilde{Q}+\tilde{F}_{g}(v)\right]=0 .
$$

A free-running steady-state detonation propagates at the minimum allowed detonation speed, the ChapmanJouguet (CJ) speed $\tilde{D}_{\mathrm{CJ}}$. At the CJ state the function on the left-hand side of the above equation must have a double zero, requiring its derivative to also vanish, yielding

$$
2 v-2\left(\frac{1+\omega_{g}}{2+\omega_{g}}\right)+\left(\frac{2 \omega_{g}}{2+\omega_{g}}\right) \frac{\tilde{v}_{s_{0}}}{\tilde{D}_{0}^{2}} \tilde{F}_{g}^{\prime}(v)=0 .
$$

The simultaneous solution of the last two equations yields the values of $v_{\mathrm{CJ}}$ and $\tilde{D}_{\mathrm{CJ}}$. Carrying the details through, the solution of each of the last two equations for $\tilde{D}_{0}$ yields the pair

$$
\begin{aligned}
& \left(\frac{2+\omega_{g}}{2 \omega_{g}}\right) \frac{\tilde{D}_{0}^{2}}{\tilde{v}_{s_{0}}}=-\frac{\tilde{Q}+\tilde{F}_{g}(v)}{(v-1)\left(v-\frac{\omega_{g}}{2+\omega_{g}}\right)}, \\
& \left(\frac{2+\omega_{g}}{2 \omega_{g}}\right) \frac{\tilde{D}_{0}^{2}}{\tilde{v}_{s_{0}}}=-\frac{\tilde{F}_{g}^{\prime}(v)}{2\left(v-\frac{1+\omega_{g}}{2+\omega_{g}}\right)} .
\end{aligned}
$$

Upon equating the right-hand sides and rearranging, we find that $v_{\mathrm{CJ}}$ must be the root of

$$
\tilde{F}_{g}^{\prime}(v)-\left[\tilde{Q}+\tilde{F}_{g}(v)\right]\left\{\frac{1}{v-1}+\frac{1}{v-\frac{\omega_{g}}{2+\omega_{g}}}\right\}=0 .
$$

Then from (32), we get $\tilde{D}_{\mathrm{CJ}}$ as

$$
\tilde{D}_{\mathrm{CJ}}^{2}=-\tilde{v}_{s_{0}}\left(\frac{2 \omega_{g}}{2+\omega_{g}}\right) \frac{\tilde{Q}+\tilde{F}_{g}\left(v_{\mathrm{CJ}}\right)}{\left(v_{\mathrm{CJ}}-1\right)\left(v_{\mathrm{CJ}}-\frac{\omega_{g}}{2+\omega_{g}}\right)} .
$$

Finally, (23) and (24) yield, respectively,

$$
\tilde{U}_{\mathrm{CJ}}=\tilde{D}_{0} v_{\mathrm{CJ}}, \quad \tilde{p}_{\mathrm{CJ}}=\frac{\tilde{D}_{0}^{2}}{\tilde{v}_{s_{0}}}\left(1-v_{\mathrm{CJ}}\right) .
$$




\section{Scaling and Nondimensionalization}

With the CJ wave established as above, we employ its properties to select reference quantities as follows.

$\begin{array}{ll}\text { Volume } & \tilde{v}_{\text {ref }}=\tilde{v}_{s_{0}}, \\ \text { Velocity } & \tilde{u}_{\text {ref }}=\tilde{D}_{\mathrm{CJ}}, \\ \text { Pressure } & \tilde{p}_{\text {ref }}=\tilde{D}_{\mathrm{CJ}}^{2} / \tilde{v}_{s_{0}}, \\ \text { Energy } & \tilde{e}_{\text {ref }}=\tilde{D}_{\mathrm{CJ}}^{2}, \\ \text { Temperature } & \tilde{T}_{\text {ref }} \text { (to be specified later), } \\ \text { Time } & \tilde{t}_{\text {ref }} \text { (to be specified later), } \\ \text { Length } & \tilde{\ell}_{\text {ref }}=\tilde{t}_{\text {ref }} \tilde{D}_{\mathrm{CJ}}, \\ \text { Specific heat } & \tilde{C}_{\text {ref }}=\tilde{p}_{\text {ref }} / \tilde{T}_{\text {ref }} .\end{array}$

Then, constituent specific energies scale as

$$
e_{i}=\frac{\tilde{e}_{i}}{\tilde{u}_{\mathrm{ref}}^{2}}=\frac{\tilde{v}_{s_{0}}}{\tilde{D}_{\mathrm{CJ}}^{2}} \tilde{E}_{i}=\frac{1}{\tilde{p}_{\mathrm{ref}}} \tilde{E}_{i},
$$

where (5) has been employed. We note, in particular, that $\tilde{p}_{\text {ref }}$ emerges as the unit of energy per unit volume. The reaction rate is scaled as

$$
\mathcal{R}=\tilde{t}_{\text {ref }} \tilde{\mathcal{R}}
$$

\subsection{Scaled Unsteady Equations}

The governing equations (17) assume the dimensionless form

$$
\mathbf{u}_{t}+\mathbf{f}(\mathbf{u})_{x}+\mathbf{g}(\mathbf{u})_{y}=\mathbf{h}(\mathbf{u}),
$$

where

$$
\mathbf{u}=\left[\begin{array}{c}
\rho \\
\rho u \\
\rho w \\
\rho \mathcal{E} \\
\rho \lambda
\end{array}\right], \quad \mathbf{f}=\left[\begin{array}{c}
\rho u \\
\rho u^{2}+p \\
\rho u w \\
u(\rho \mathcal{E}+p) \\
\rho u \lambda
\end{array}\right], \quad \mathbf{g}=\left[\begin{array}{c}
\rho w \\
\rho w u \\
\rho w^{2}+p \\
v(\rho \mathcal{E}+p) \\
\rho v \lambda
\end{array}\right], \quad \mathbf{h}=\left[\begin{array}{c}
0 \\
0 \\
0 \\
0 \\
\rho R
\end{array}\right]
$$

\subsection{Scaled Equations of State}

The dimensionless versions of the equations of state for the two constituents, equations (1)-(7), are as follows. Mechanical:

$$
\begin{aligned}
e_{s} & =\frac{p_{s} v_{s}}{\omega_{s}}-F_{s}\left(v_{s}\right)+F_{s}(1), \\
e_{g} & =\frac{p_{g} v_{g}}{\omega_{g}}-F_{g}\left(v_{g}\right)-Q .
\end{aligned}
$$

Here,

$$
F_{i}=\frac{\tilde{F}_{i}}{\tilde{p}_{\text {ref }}}, \quad A_{i}=\frac{\tilde{A}_{i}}{\tilde{p}_{\text {ref }}}, \quad B_{i}=\frac{\tilde{B}_{i}}{\tilde{p}_{\text {ref }}}, \quad Q=\frac{\tilde{Q}}{\tilde{p}_{\text {ref }}} .
$$

Thermal:

$$
\begin{aligned}
& p_{s}=\frac{\omega_{s}}{v_{s}}\left[C_{s} T_{s}+G_{s}\left(v_{s}\right)+F_{s}\left(v_{s}\right)\right], \\
& p_{g}=\frac{\omega_{g}}{v_{g}}\left[C_{g} T_{g}+G_{g}\left(v_{g}\right)+F_{g}\left(v_{g}\right)\right] .
\end{aligned}
$$


Here,

$$
\begin{aligned}
& F_{s}(V)=A_{s}\left(\frac{V}{\omega_{s}}-\frac{1}{R_{1_{s}}}\right) \exp \left(-R_{1_{s}} V\right)+B_{s}\left(\frac{V}{\omega_{s}}-\frac{1}{R_{2_{s}}}\right) \exp \left(-R_{2_{s}} V\right), \\
& F_{g}(V)=A_{g}\left(\frac{V}{\omega_{g}}-\frac{1}{R_{1_{g}}}\right) \exp \left(-R_{1_{g}} V\right)+B_{g}\left(\frac{V}{\omega_{g}}-\frac{1}{R_{2_{g}}}\right) \exp \left(-R_{2_{g}} V\right),
\end{aligned}
$$

and

$$
\begin{aligned}
G_{s}(V) & =\frac{A_{s}}{R_{1_{s}}} \exp \left(-R_{1_{s}} V\right)+\frac{B_{s}}{R_{2_{s}}} \exp \left(-R_{2_{s}} V\right), \\
G_{g}(V) & =\frac{A_{g}}{R_{1_{g}}} \exp \left(-R_{1_{g}} V\right)+\frac{B_{g}}{R_{2_{g}}} \exp \left(-R_{2_{g}} V\right) .
\end{aligned}
$$

The mixture energy, scaled version of (14) and mindful of pressure equilibrium, is

$$
e=p\left\{\frac{1-\lambda}{\omega_{s}} v_{s}+\frac{\lambda}{\omega_{g}} v_{g}\right\}+(1-\lambda)\left[F_{s}(1)-F_{s}\left(v_{s}\right)\right]-\lambda\left[F_{g}\left(v_{g}\right)+Q\right]
$$

The temperatures, scaled versions of (3) and (4) and mindful of pressure equilibrium, are

$$
\begin{aligned}
T_{s} & =\frac{1}{C_{s}}\left[\frac{p v_{s}}{\omega_{s}}-F_{s}\left(v_{s}\right)-G_{s}\left(v_{s}\right)\right] \\
T_{g} & =\frac{1}{C_{g}}\left[\frac{p v_{g}}{\omega_{g}}-F_{g}\left(v_{g}\right)-G_{g}\left(v_{g}\right)\right] .
\end{aligned}
$$

The condition of temperature equilibrium, $T_{s}=T_{g}=T$, is given by

$$
p\left\{\frac{v_{s}}{\omega_{s} C_{s}}-\frac{v_{g}}{\omega_{g} C_{g}}\right\}-\frac{1}{C_{s}}\left[F_{s}\left(v_{s}\right)+G_{s}\left(v_{s}\right)\right]+\frac{1}{C_{g}}\left[F_{g}\left(v_{g}\right)+G_{g}\left(v_{g}\right)\right]=0 .
$$

\subsection{Scaled Steady Equations}

The conditions of mass and momentum conservation, scaled versions of (23) and (24), are

$$
\begin{aligned}
U & =f v, \\
p & =f^{2}(1-v),
\end{aligned}
$$

while the energy balance, scaled version of $(22)$, is

$$
e+p v+\frac{1}{2} U^{2}=e_{0}+\frac{1}{2} f^{2} .
$$

The degree of overdrive $f$ is given by

$$
f=\frac{\tilde{D}_{0}}{\tilde{D}_{\mathrm{CJ}}}
$$

\subsection{The Steady Structure}

The structure problem consists of solving the six equations representing mass balance (52), momentum balance (53), energy balance (54), mixture energy (48), temperature equilibrium (51) and mixture volume (16) for the six state variables $U, v, p, e, v_{s}$ and $v_{g}$ as functions of $\lambda$. Then, the equilibrium temperature $T$ can be computed from (49) or (50).

This procedure can be simplified by using mass balance (52), momentum balance (53) and energy balance (54) to first eliminate $U, p$ and $e$. This leaves three equations for $v, v_{s}$ and $v_{g}$. Of these, the mixture 


\begin{tabular}{|c|c|c|}
\hline JWL parameters & Unreacted & Products \\
\hline$\tilde{A}(\mathrm{~Pa})$ & $778.1 \mathrm{e} 11$ & $14.8105 \mathrm{e} 11$ \\
\hline$\tilde{B}(\mathrm{~Pa})$ & $-0.05031 \mathrm{e} 11$ & $0.6379 \mathrm{e} 11$ \\
\hline$R_{1}$ & 11.3 & 6.2 \\
\hline$R_{2}$ & 1.13 & 2.2 \\
\hline$\omega$ & 0.8938 & 0.50 \\
\hline$\tilde{Q}(\mathrm{~Pa}-\mathrm{cc} / \mathrm{cc})$ & & $0.069 \mathrm{e} 11$ \\
\hline$\tilde{C}(\mathrm{~Pa} / \mathrm{K})$ & $2.487 \mathrm{e} 6$ & $1.0 \mathrm{e} 6$ \\
\hline$\tilde{\rho}_{0}\left(\mathrm{~kg} / \mathrm{m}^{3}\right)$ & 1905 & \\
\hline
\end{tabular}

Table 1: EOS data for the plastic-bonded explosive LX-17.

volume equation (16) is one. The other two are obtained from the mixture energy equation (48) and the temperature equilibrium equation (51). After some manipulations these equations are found to be

$$
\begin{array}{r}
f^{2}(1-v)\left\{\frac{1-\lambda}{\omega_{s}} v_{s}+\frac{\lambda}{\omega_{g}} v_{g}+\frac{1}{2} v-\frac{1}{2}\right\}+(1-\lambda)\left[F_{s}(1)-F_{s}\left(v_{s}\right)\right]-\lambda\left[F_{g}\left(v_{g}\right)+Q\right]=0 \\
f^{2}(1-v)\left\{\frac{v_{s}}{\omega_{s} C_{s}}-\frac{v_{g}}{\omega_{g} C_{g}}\right\}-\frac{1}{C_{s}}\left[F_{s}\left(v_{s}\right)+G_{s}\left(v_{s}\right)\right]+\frac{1}{C_{g}}\left[F_{g}\left(v_{g}\right)+G_{g}\left(v_{g}\right)\right]=0 .
\end{array}
$$

We can use the mixture-volume equation (16) to eliminate either $v_{s}$ (if $\lambda$ is not near unity) or $v_{g}$ (if $\lambda$ is not near zero) from the above pair of equations. For given $\lambda$, this pair can then be solved for $v$, and either $v_{g}$ or $v_{s}$. The remaining state variables can then be solved for by appealing to (52), (53) and (54). It is assumed, of course, that the overdrive factor $f$ is prescribed. Finally, with the structure known in terms of $\lambda$, the planar, steady version of the rate equation in (38) yields the spatial distribution of $\lambda$ in the reaction zone.

We note, for later use, that the volume $v^{\mathrm{S}}$ immediately behind the lead shock propagating into ambient, unreacted explosive can be related to the overdrive $f$ by setting $\lambda=0$ in (56) above. The result is

$$
f^{2}=\frac{2 \omega_{s}\left[F_{s}(1)-F_{s}\left(v_{s}\right)\right]}{\left(2+\omega_{s}\right)\left(v^{\mathrm{S}}\right)^{2}-2\left(1+\omega_{s}\right) v^{\mathrm{S}}+\omega_{s}} .
$$

\section{The Data Set}

We now introduce a data set that is representative of the explosive LX-17 (92.5\% trinitrobenzene-triamine (TATB), 7.5\% Kel-F); see Dobratz [17]. We reiterate that there does not exist in the literature a unique data set for this (or any other) explosive, and that the data depend to some extent on the experiment or configuration being modeled. The EOS and rate parameters given in Table 1 are taken from the recent paper by Tarver [11] who observes that this parameter set is appropriate for detonation propagation rather than initiation.

The corresponding CJ structure is characterized by the following CJ and von Neumann states:

$$
\begin{array}{ll}
D_{\mathrm{CJ}}=7679.9473 \mathrm{~m} / \mathrm{s}, & \\
p_{\mathrm{CJ}}=0.2699980 \mathrm{e} 11 \mathrm{~Pa}, & p_{\mathrm{N}}=0.348236 \mathrm{e} 11 \mathrm{~Pa}, \\
\rho_{0} / \rho_{\mathrm{CJ}}=0.759703, & \rho_{0} / \rho_{\mathrm{N}}=0.69007, \\
u_{\mathrm{CJ}}=1845.4711 \mathrm{~m} / \mathrm{s} \text { (lab frame), } & u_{\mathrm{N}}=2380.2367 \mathrm{~m} / \mathrm{s} \text { (lab frame) }
\end{array}
$$

The corresponding reference quantities, as defined at the beginning of section 4 , are

$$
\begin{array}{lll}
\tilde{D}_{\mathrm{CJ}}=7679.9473 \mathrm{~m} / \mathrm{s}, & \tilde{v}_{s 0}=1 / 1905 \mathrm{~kg} / \mathrm{m}^{3}, & \tilde{p}_{\text {ref }}=D_{\mathrm{CJ}}^{2} / \tilde{v}_{s 0}=1.1235993 \mathrm{e} 11 \mathrm{~Pa} \\
\tilde{T}_{\text {ref }}=300^{\circ} \mathrm{K}, & \tilde{t}_{\text {ref }}=10^{-6} \mathrm{~s}, & \tilde{\ell}_{\text {ref }}=\tilde{D}_{\mathrm{CJ}} \tilde{t}_{\text {ref }}=0.76799473 \mathrm{~cm} .
\end{array}
$$




\begin{tabular}{|c|c|}
\hline Parameter & Value \\
\hline$\tilde{I},\left(s^{-1}\right)$ & $4.0 \mathrm{e} 12$ \\
\hline$b$ & 0.667 \\
\hline$a$ & 0.22 \\
\hline$x$ & 7.0 \\
\hline$\left.\tilde{G}_{1},\left(10^{11} P a\right)^{-y} s^{-1}\right)$ & $4500 \mathrm{e} 6$ \\
\hline$c$ & 0.667 \\
\hline$d$ & 1 \\
\hline$y$ & 3 \\
\hline$\left.\tilde{G}_{2},\left(10^{11} P a\right)^{-z} s^{-1}\right)$ & $30 \mathrm{e} 6$ \\
\hline$e$ & 0.667 \\
\hline$g$ & 0.667 \\
\hline$z$ & 1 \\
\hline$\lambda_{\text {ig, } \max }$ & 0.02 \\
\hline$\lambda_{G_{1}, \max }$ & 0.8 \\
\hline$\lambda_{G_{2}, \min }$ & 0.8 \\
\hline
\end{tabular}

Table 2: Rate data.

Then, the scaled CJ and von Neumann states emerge as

$$
\begin{array}{ll}
p_{\mathrm{CJ}}=0.24029737, & p_{\mathrm{N}}=0.30992878 \\
v_{\mathrm{CJ}}=0.75970263, & v_{\mathrm{N}}=0.69007122 \\
u_{\mathrm{CJ}}=0.24029737 . &
\end{array}
$$

Table 2 gives the parameters for the rate law. The Hugoniot diagrams and the structure of the planar CJ reaction zone are shown in Figure 1. The crossing of the Hugoniot curves in the upper left corner of the figure is indicative of the unreliability of the equation of state at very high pressures. The stiffness of the reaction rate, which varies by two orders of magnitude, is worth noting.

\section{Numerical Method}

With the steady detonation structure in hand, we now turn to a computational study of unsteady detonation evolution. We begin by providing a brief description of the numerical procedure used to compute solutions of the two-dimensional ignition-and-growth model, and a demonstration of the grid convergence for a representative calculation. Solutions of the one-dimensional equations are obtained using a straightforward reduction of the two-dimensional scheme; three-dimensional axi-symmetric problems are handled by including a geometric source term.

A numerical approximation of the governing equations is carried out using a finite-volume discretization on composite overlapping grids following the approach described in Henshaw and Schwendeman [14]. A composite grid consists of collection of logically rectangular, curvilinear component grids that overlap where they meet and cover the domain of interest. At the overlap, the numerical solution is communicated across the component grids using interpolation. This grid construction is fairly general and may be used to handle complex geometric configurations, including the corner-turning problems considered in this paper. Blockstructured adaptive mesh refinement (AMR) is used to locally increase the grid resolution near shocks, contacts and detonations. The basic approach follows that introduced by Berger and Oliger [18] but extended to overlapping grids as discussed in [14].

Each component grid, whether on the base level or on a refined-grid level, is defined by a mapping from physical space $(x, y)$ to the unit square in a computational space $(r, s)$, say. In computational space, $(17)$ becomes

$$
\mathbf{U}_{t}+\mathbf{F}(\mathbf{U})_{r}+\mathbf{G}(\mathbf{U})_{s}=\mathbf{H}(\mathbf{U}),
$$




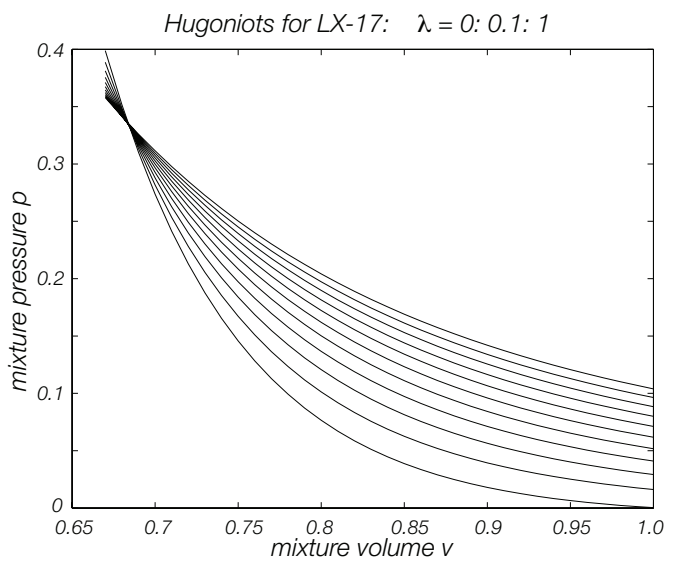

(a)

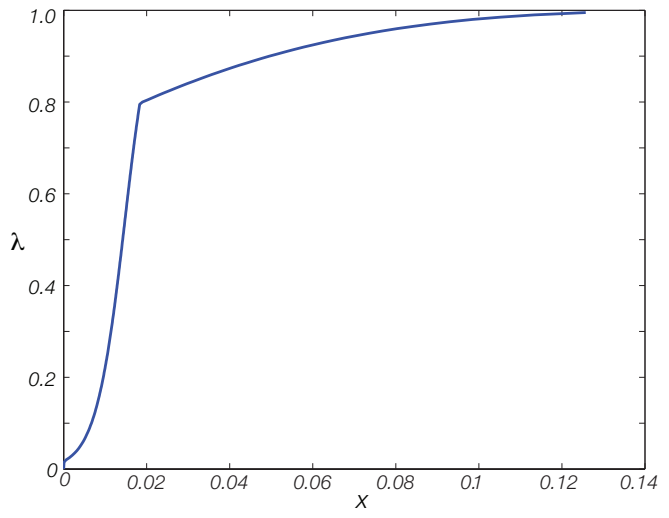

(b)

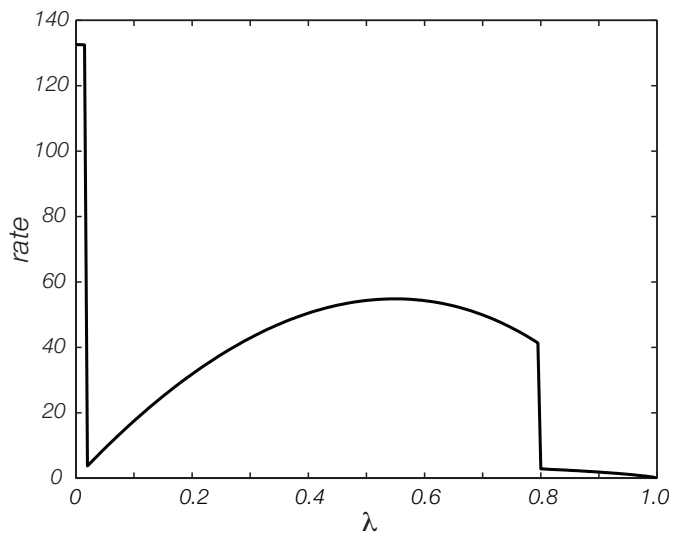

(c)

Figure 1: (a) Hugoniot curves, (b) reaction zone profile and (c) reaction rate versus $\lambda$ for LX-17. The variation of the reaction rate over two orders of magnitude observed in plot (b) and (c) is noteworthy.

where

$$
\mathbf{U}=J \mathbf{u}, \quad \mathbf{F}=y_{s} \mathbf{f}-x_{s} \mathbf{g}, \quad \mathbf{G}=-y_{r} \mathbf{f}+x_{r} \mathbf{g}, \quad \mathbf{H}=J \mathbf{h},
$$

and

$$
J=\left|\frac{\partial(x, y)}{\partial(r, s)}\right| .
$$

The partial derivatives, $y_{s}, x_{s}$, etc., and the Jacobian of the mapping are known for each component grid. The mapped equation (59) is discretized on a uniform grid $\left(r_{i}, s_{j}\right)$ with mesh spacings $(\Delta r, \Delta s)$ using the conservative scheme

$$
\mathbf{U}_{i, j}^{n+1}=\mathbf{U}_{i, j}^{n}-\frac{\Delta t}{\Delta r}\left(\mathbf{F}_{i+1 / 2, j}^{n+1 / 2}-\mathbf{F}_{i-1 / 2, j}^{n+1 / 2}\right)-\frac{\Delta t}{\Delta s}\left(\mathbf{G}_{i, j+1 / 2}^{n+1 / 2}-\mathbf{G}_{i, j-1 / 2}^{n+1 / 2}\right)+\Delta t \mathbf{H}_{i, j}^{n+1 / 2},
$$

where $\mathbf{U}_{i, j}^{n}$ is an approximation of the cell average of $\mathbf{U}$ at a time $t_{n}, \mathbf{F}_{i \pm 1 / 2, j}^{n+1 / 2}$ and $\mathbf{G}_{i, j \pm 1 / 2}^{n+1 / 2}$ are numerical fluxes, and $\mathbf{H}_{i, j}^{n+1 / 2}$ is a numerical source term. The numerical fluxes are computed using a second-order extension of Godunov's method with a Roe-type approximate Riemann solver. The source term is computed using a second-order Runge-Kutta error-control scheme. Details are provided in [14] for the case of an ideal gas. Here we focus our attention on the extension required to treat the mixture JWL equation of state used in the ignition-and-growth model. 


\subsection{Riemann solver}

The approximate Riemann solver is based on the exact solution of a linearized Riemann problem. For the fluxes $\mathbf{F}_{i \pm 1 / 2, j}^{n+1 / 2}$, for example, we consider the Riemann problem

$$
\mathbf{u}_{t}+\bar{A} \mathbf{u}_{r}=0, \quad|r|<\infty, \quad t>0
$$

with initial conditions

$$
\mathbf{u}(r, 0)= \begin{cases}\mathbf{u}_{L}, & \text { if } r<0 \\ \mathbf{u}_{R}, & \text { if } r>0\end{cases}
$$

where $\bar{A}=\mathbf{F}_{\mathbf{U}}(\overline{\mathbf{u}})$ and $\overline{\mathbf{u}}$ is an averaged state depending on $\mathbf{u}_{L}$ and $\mathbf{u}_{R}$. The left and right states come from slope-limited corrections of cell averages as described in [14] and are assumed to be known. Further, let us assume for the moment that $\overline{\mathbf{u}}$ is known. The solution of the Riemann problem may be expressed in terms of the eigenvalues and eigenvectors of $\mathbf{F}_{\mathbf{U}}$. We first define the quantities

$$
\alpha=\frac{y_{s}}{\sqrt{y_{s}^{2}+x_{s}^{2}}}, \quad \beta=\frac{-x_{s}}{\sqrt{y_{s}^{2}+x_{s}^{2}}}, \quad c=\frac{\sqrt{y_{s}^{2}+x_{s}^{2}}}{J},
$$

in terms of the known metrics and Jacobian of the mapping. The eigenvalues $\bar{\mu}_{i}, i=1, \ldots, 5$, of $\bar{A}$ are then given by

$$
\bar{\mu}_{1}=c\left(\bar{u}_{n}-\bar{a}\right), \quad \bar{\mu}_{2}=\bar{\mu}_{3}=\bar{\mu}_{4}=c \bar{u}_{n}, \quad \bar{\mu}_{5}=c\left(\bar{u}_{n}+\bar{a}\right)
$$

where

$$
\bar{u}_{n}=\alpha \bar{u}+\beta \bar{w}, \quad \bar{a}=\left[\bar{p}_{\rho}+\left(\bar{h}-\frac{1}{2}\left(\bar{u}^{2}+\bar{w}^{2}\right)\right) \bar{p}_{\rho e}+\bar{\lambda} \bar{p}_{\rho \lambda}\right]^{1 / 2},
$$

Here, $\bar{u}_{n}$ is the component of the velocity normal to the curve $r=$ constant, $\bar{a}$ is the sound speed, and $\bar{h}$ is the total enthalpy. For this analysis, it is convenient to regard $p$ as a function of $\rho, \rho e$ and $\rho \lambda$. This function is defined implicitly by the mixture JWL equation of state, and its numerical treatment is discussed in the next subsection. The eigenvectors $\overline{\mathbf{r}}_{i}, i=1, \ldots, 5$, are the columns of the matrix

$$
\bar{R}=\left(\begin{array}{ccccc}
1 & 0 & 1 & 0 & 1 \\
\bar{u}-\alpha \bar{a} & -\beta & \bar{u} & 0 & \bar{u}+\alpha \bar{a} \\
\bar{w}-\beta \bar{a} & \alpha & \bar{w} & 0 & \bar{w}+\beta \bar{a} \\
\bar{h}-\bar{u}_{n} \bar{a} & \alpha \bar{w}-\beta \bar{u} & \frac{1}{2}\left(\bar{u}^{2}+\bar{w}^{2}\right)-\bar{p}_{\rho} / \bar{p}_{\rho e} & -\bar{p}_{\rho \lambda} / \bar{p}_{\rho e} & \bar{h}+\bar{u}_{n} \bar{a} \\
\bar{\lambda} & 0 & 0 & 1 & \bar{\lambda}
\end{array}\right)
$$

The numerical flux is associated with the exact flux $\mathbf{F}_{0}$ of solution of (61) along the line $r=0$, which is given by

$$
\mathbf{F}_{0}= \begin{cases}\mathbf{F}\left(\mathbf{u}_{L}\right), & \text { if } \bar{\mu}_{1}>0 \\ \mathbf{F}\left(\mathbf{u}_{L}\right)+\bar{\kappa}_{1} \bar{\mu}_{1} \overline{\mathbf{r}}_{1}, & \text { if } \bar{\mu}_{1}<0 \text { and } \bar{\mu}_{2}>0 \\ \mathbf{F}\left(\mathbf{u}_{R}\right)-\bar{\kappa}_{5} \bar{\mu}_{5} \overline{\mathbf{r}}_{5}, & \text { if } \bar{\mu}_{2}<0 \text { and } \bar{\mu}_{5}>0 \\ \mathbf{F}\left(\mathbf{u}_{R}\right), & \text { if } \bar{\mu}_{5}<0\end{cases}
$$

where $\bar{\kappa}_{1}$ and $\bar{\kappa}_{5}$ are the first and last components of the vector $\bar{R}^{-1}\left(\mathbf{u}_{R}-\mathbf{u}_{L}\right)$, respectively.

It remains to specify an averaged state $\overline{\mathbf{u}}$ in terms of the given left and right states $\mathbf{u}_{L}$ and $\mathbf{u}_{R}$. This averaged state is constructed following the prescription given by Roe [19] and modified for the mixture JWL equation following the discussion given by Glaister [20]. According to the Roe prescription, $\overline{\mathbf{u}}$ is a state which satisfies the system

$$
\mathbf{F}\left(\mathbf{u}_{R}\right)-\mathbf{F}\left(\mathbf{u}_{L}\right)=\bar{A}\left(\mathbf{u}_{R}-\mathbf{u}_{L}\right), \quad \bar{A}=\mathbf{F}_{\mathbf{U}}(\overline{\mathbf{u}}) .
$$

In order to determine $\overline{\mathbf{u}}$ from (65) it is simplest to take $\alpha=1$ and $\beta=0$ in $\bar{A}$ and then interpret the velocity components $(\bar{u}, \bar{w})$ as the components normal and tangential to the curve $r=$ constant, 
respectively. (Once $(\bar{u}, \bar{w})$ are determined, then a simple rotation recovers the velocity components in (62) and (63).) The first component of (65) is satisfied identically, while the second component is

$$
\begin{gathered}
\Delta\left(\rho u^{2}\right)+\Delta p=\left(-\bar{u}^{2}+\bar{p}_{\rho}+\bar{p}_{\rho e} \frac{1}{2}\left(\bar{u}^{2}+\bar{w}^{2}\right)\right) \Delta \rho+\left(2 \bar{u}-\bar{u} \bar{p}_{\rho e}\right) \Delta(\rho u) \\
+\left(-\bar{w}_{\rho e}\right) \Delta(\rho w)+\left(\bar{p}_{\rho e}\right) \Delta E+\left(\bar{p}_{\rho \lambda}\right) \Delta(\rho \lambda),
\end{gathered}
$$

where $\Delta \rho=\rho_{R}-\rho_{L}, \Delta(\rho u)=\rho_{R} u_{R}-\rho_{L} u_{L}$, etc., are known. Following [19], we set

$$
\bar{u}=\frac{\sqrt{\rho_{L}} u_{L}+\sqrt{\rho_{R}} u_{R}}{\sqrt{\rho_{L}}+\sqrt{\rho_{R}}}, \quad \bar{w}=\frac{\sqrt{\rho_{L}} w_{L}+\sqrt{\rho_{R}} w_{R}}{\sqrt{\rho_{L}}+\sqrt{\rho_{R}}},
$$

so that

$$
\Delta\left(\rho u^{2}\right)-2 \bar{u} \Delta(\rho u)+\bar{u}^{2} \Delta \rho=\Delta\left(\rho w^{2}\right)-2 \bar{w} \Delta(\rho w)+\bar{w}^{2} \Delta \rho=0,
$$

and (66) reduces to

$$
\Delta p=\left(\bar{p}_{\rho}\right) \Delta \rho+\left(\bar{p}_{\rho e}\right) \Delta(\rho e)+\left(\bar{p}_{\rho \lambda}\right) \Delta(\rho \lambda) .
$$

Let us assume for the moment that $\bar{p}_{\rho}, \bar{p}_{\rho e}$ and $\bar{p}_{\rho \lambda}$ are known such that (68) is satisfied. If true, it can be shown that the remaining components of (65) are satisfied for the choices given in (67) and

$$
\bar{h}=\frac{\sqrt{\rho_{L}} h_{L}+\sqrt{\rho_{R}} h_{R}}{\sqrt{\rho_{L}}+\sqrt{\rho_{R}}}, \quad \bar{\lambda}=\frac{\sqrt{\rho_{L}} \lambda_{L}+\sqrt{\rho_{R}} \lambda_{R}}{\sqrt{\rho_{L}}+\sqrt{\rho_{R}}} .
$$

The final task involves a specification of $\bar{p}_{\rho}, \bar{p}_{\rho e}$ and $\bar{p}_{\rho \lambda}$ in terms of known quantities in the left and right states so that (68) is satisfied. If we regard the three partial derivatives of $p$ as coordinates in a three-dimensional space, then we may view the problem geometrically as finding the point $\left(\bar{p}_{\rho}, \bar{p}_{\rho e}, \bar{p}_{\rho \lambda}\right)$ on the plane given by (68). From this point of view, it is clear that the choice is not unique. Glaister offers a choice in [20] for the non-reactive Euler equations with a general equation of state. An analogous choice for the reacitve case has the difficulty of possibly evaluating the partial derivatives of $p$ at values where $\lambda$ is not in $[0,1]$. We use an alternate choice which begins with the arithmetic means

$$
\hat{p}_{\rho}=\frac{1}{2}\left[\left(p_{\rho}\right)_{L}+\left(p_{\rho}\right)_{R}\right], \quad \hat{p}_{\rho e}=\frac{1}{2}\left[\left(p_{\rho e}\right)_{L}+\left(p_{\rho e}\right)_{R}\right], \quad \hat{p}_{\rho \lambda}=\frac{1}{2}\left[\left(p_{\rho \lambda}\right)_{L}+\left(p_{\rho \lambda}\right)_{R}\right] .
$$

Then, $\bar{p}_{\rho}, \bar{p}_{\rho e}$ and $\bar{p}_{\rho \lambda}$ are taken to be the point on the plane with minimum Euclidean distance to the arithmetics means. This choice gives

$$
\bar{p}_{\rho}=\hat{p}_{\rho}+\theta \Delta \rho, \quad \bar{p}_{\rho e}=\hat{p}_{\rho e}+\theta \Delta(\rho e), \quad \bar{p}_{\rho \lambda}=\hat{p}_{\rho \lambda}+\theta \Delta(\rho \lambda),
$$

where

$$
\theta=\frac{\Delta p-\left(\hat{p}_{\rho}\right) \Delta \rho-\left(\hat{p}_{\rho e}\right) \Delta(\rho e)-\left(\hat{p}_{\rho \lambda}\right) \Delta(\rho \lambda)}{\left[(\Delta \rho)^{2}+(\Delta(\rho e))^{2}+(\Delta(\rho \lambda))^{2}\right]^{1 / 2}} .
$$

In uniform regions of flow where the left and right states are the same so that $\Delta \rho=\Delta(\rho e)=\Delta(\rho \lambda)=0$, we assign $\theta=0$ to avoid division by zero.

\subsection{Evaluation of the mixture JWL equation of state}

The mixture pressure $p$ and the species volumes $v_{s}$ and $v_{g}$ are defined implicitly by the (scaled) mixture volume and energy equations, (16) and (48), respectively, and by the temperature equilibrium equation, (51), for a given density $\rho=1 / v$, internal energy $e$, and reaction progress $\lambda$. These equations require a numerical treatment, in generaly, to determine $p$ and its derivatives $p_{\rho}, p_{\rho e}$ and $p_{\rho \lambda}$ as needed for the various formulas for numerical flux discussed above.

First, we note that in the limiting cases $\lambda=0$ and $\lambda=1, p$ may be found explicitly. For example, if $\lambda=0$, then $v_{s}=v=1 / \rho$ from (16) and $p$ is found from the mechanical equation of state (40) for the solid using $e_{s}=e$. (The temperature equilibrium equation would determine $v_{g}$ but this quantity is not needed 
for this limiting case.) Similarly, if $\lambda=1$, then $v_{g}=v=1 / \rho$ and $p$ is found from (41). In our numerical scheme, we use these explicit formulas for $p$ if $\lambda \leq \lambda_{0}$ or $\lambda \geq \lambda_{1}$, where $\lambda_{0}=0.001$ and $\lambda_{1}=0.999$.

A numerical method is needed to determine $p$ when $\lambda$ is away from 0 and 1 . For this case, we first eliminate $p$ from (48) and (51) which yields the equation

$$
\begin{aligned}
N\left(v_{s}, v_{g}\right)= & \left\{\frac{v_{s}}{\omega_{s} C_{s}}-\frac{v_{g}}{\omega_{g} C_{g}}\right\}\left\{e+(1-\lambda)\left[F_{s}\left(v_{s}\right)-F_{s}(1)\right]+\lambda\left[F_{g}\left(v_{g}\right)+Q\right]\right\} \\
& -\left\{\frac{1-\lambda}{\omega_{s}} v_{s}+\frac{\lambda}{\omega_{g}} v_{g}\right\}\left\{\frac{1}{C_{s}}\left[F_{s}\left(v_{s}\right)+G_{s}\left(v_{s}\right)\right]-\frac{1}{C_{g}}\left[F_{g}\left(v_{g}\right)+G_{g}\left(v_{g}\right)\right]\right\}=0
\end{aligned}
$$

for $v_{s}$ and $v_{g}$. If $\lambda_{0}<\lambda<0.5$, then we eliminate $v_{s}$ using (16), and employ Newton's method to determine $v_{g}$ such that $|N|<\delta$, where $\delta=10^{-8}$. If $0.5 \leq \lambda<\lambda_{1}$, on the other hand, we eliminate $v_{g}$ and use Newton's method to determine $v_{s}$ such that $|N|<\delta$. These iterations converge quadratically provided that the starting values for $\left(v_{s}, v_{g}\right)$ are sufficiently close to the solution. Assuming the iteration converges, $p$ may now be obtained explicitly from (48), and then its derivatives may be found from (16), (48) and (51) using implicit differentiation.

A complication involves the determination of good starting values for $\left(v_{s}, v_{g}\right)$ for the iterative procedures. Assuming that converged values for $\left(v_{s}, v_{g}\right)$ can be found for the initial state of the flow, we update and save these values on the grid as the flow evolves whenever an iteration for $p$ is needed. In this way, good starting values for $\left(v_{s}, v_{g}\right)$ are available everywhere on the grid except possibly where the solution changes rapidly. For the diffraction problems considered in this paper, the main difficulty occurred in regions of the flow where the density is very small. In these regions, starting values for $\left(v_{s}, v_{g}\right)$ from previously converged values were not always sufficiently close to the new solution for convergence of the iterations. However, when the density is small, $\left(v_{s}, v_{g}\right)$ are large so that $N\left(v_{s}, v_{g}\right)=0$ may be approximated by the linear equation

$$
\frac{v_{s}}{\omega_{s} C_{s}}-\frac{v_{g}}{\omega_{g} C_{g}}=0
$$

since $F_{s}\left(v_{s}\right), F_{g}\left(v_{g}\right), G_{s}\left(v_{s}\right)$ and $G_{g}\left(v_{g}\right)$ are small. Solving this linear equation together with (16) gives good starting values for $\left(v_{s}, v_{g}\right)$, which we found to be an effective choice in low-density regions.

\subsection{Grid convergence}

For a typical calculation, we select mesh spacings $\Delta r$ and $\Delta s$ in computational space to give mesh spacings $\Delta x \approx \Delta y \approx 0.01$ on the base-grid level in physical space. On top of the base level, two levels of refined grids are used, as needed, with refinement factor equal to 4 in both directions. Thus, the finest grids have $\Delta x \approx \Delta y \approx 0.000625$. The time step $\Delta t$ is reduced proportionately according to the CFL stability constraint (see [14]). For the steady CJ reaction zone shown in Figure 1(b), this mesh refinement would give approximately 32 grid cells on the finest level for the portion of the reaction zone governed by the ignition and first growth stages $(0 \leq \lambda \leq 0.8)$, and approximately 200 grid cells for the whole reaction zone $(0 \leq \lambda \leq 0.99$ say). We have found that this provides sufficient grid resolution for all calculations discussed in this paper. For example, Figure 2 shows shaded contours of pressure at a fixed time for detonation diffraction around a $140^{\circ}$ corner (see section 9). The behavior of the detonation is shown at the same time from calculations using three grid resolutions: the base grid only (left), the base grid with one refinement level (middle) and the base grid with two refinement levels (right). We observe that the same qualitative behavior of the flow is obtained for all three grid resolutions, but that the detonation is best represented by the finest grid resolution. We also note that there is only a slight change in the position of the detonation and its peak pressure between the finest and next to the finest grid resolutions, and away from the detonation there is very little difference in the flow between these two calculations. The views shown in Figure 2 are representative of the grid convergence for all calculations in this paper.

\section{1D Shock Initiation}

With the steady detonation structure and the numerical procedure in hand, we are now in a position to conduct a computational study of unsteady detonation evolution. We begin with the case of a detonation 

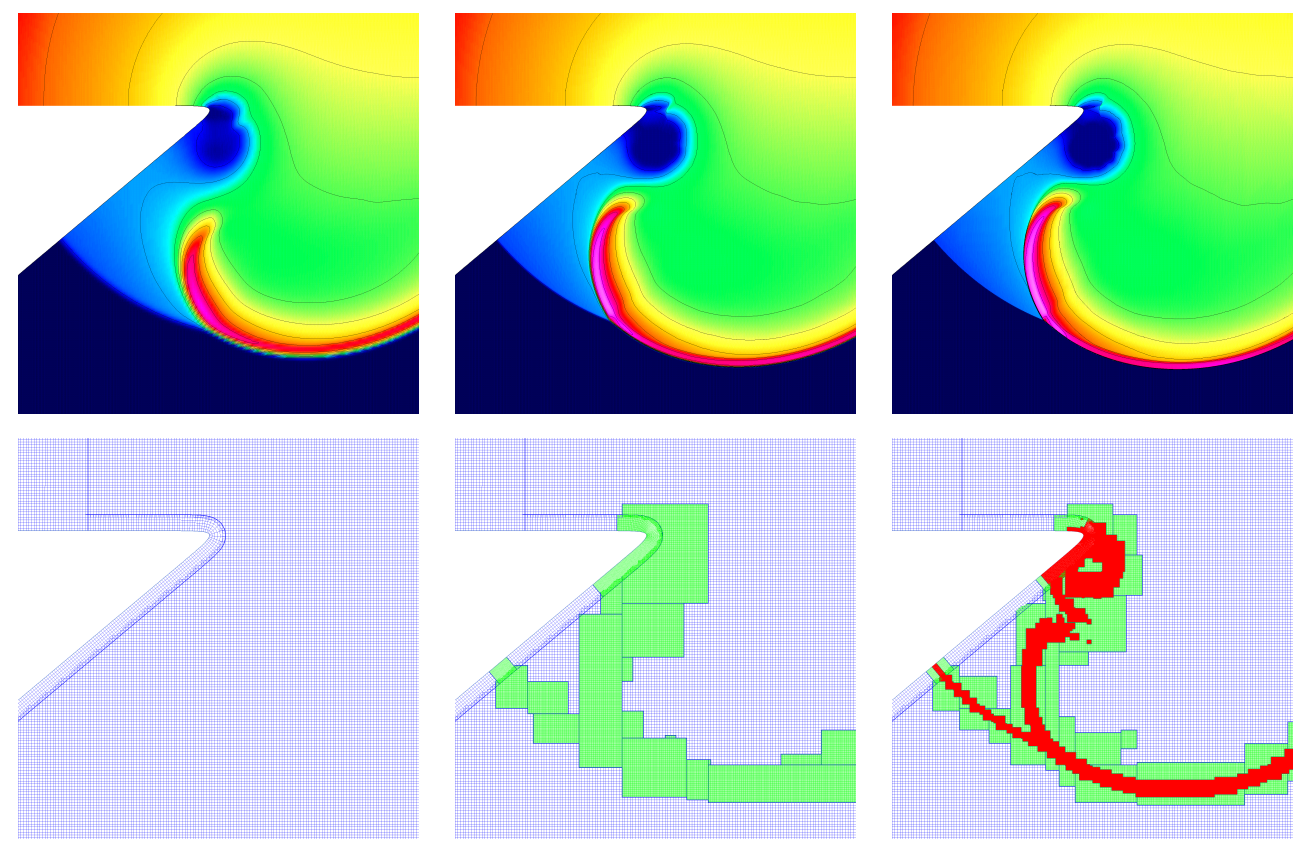

Figure 2: Shaded contours of pressure (top frames) and corresponding AMR grids (bottom frames) for detonation diffraction around a $140^{\circ}$ corner.

initiated by a planar shock. While this situation is of practical interest in its own right, the 1-D results prove to be of substantial aid in interpreting the results of 2-D simulations as well.

It is convenient from a computational viewpoint to consider the reverse impact problem, corresponding to a slab of explosive slamming impulsively into a stationary wall. The impact causes a shock to propagate into the moving explosive, and the reactive events that occur behind the lead shock are of interest. We note immediately that the shock must exceed a critical strength to switch on the initiation step in the ignition-and-growth rate law (10). The requirement is

$$
v^{\mathrm{S}}<\frac{1}{1+a}=0.8197,
$$

where $v^{\mathrm{S}}$ is the specific volume immediately behind the shock and $a(=0.22$ for LX-17 from Table 2$)$ is the compression threshold in (10). From equation (58) we find that the overdrive factor

$$
f=\frac{\tilde{D}}{\tilde{D}_{\mathrm{CJ}}}
$$

must have the value $f=0.5701$ to reach the compression threshold. This is also the upstream speed, i.e., the speed with which the ambient material enters the shock in a shock-fixed frame. The speed downstream of the shock in the same frame is

$$
U^{\mathrm{S}}=f v^{\mathrm{S}}=0.4673,
$$

and the downstream pressure,

$$
p^{\mathrm{S}}=f^{2}\left(1-v^{\mathrm{S}}\right)=0.0586 .
$$

In a wall-fixed frame, the downstream speed is zero, the shock speed $f-u^{\mathrm{S}}$ and the upstream (impact) speed $-u^{\mathrm{S}}$. In dimensional terms, $\tilde{u}^{\mathrm{S}}=u^{\mathrm{S}} \tilde{D}_{\mathrm{CJ}}=0.7895 \mathrm{~km} / \mathrm{s}$ and $\tilde{p}^{\mathrm{S}}=\tilde{p}_{\text {ref }} p^{\mathrm{S}}=6.5843 \mathrm{GPa}$.

We shall consider two representative shock-initiation cases: (i) a low-speed case and (ii) a high-speed case. The former is taken to be $u^{\mathrm{S}}=0.14$, corresponding to $\tilde{u}^{\mathrm{S}}=1.0752 \mathrm{~km} / \mathrm{s}$ and the latter $u^{\mathrm{S}}=0.1888$, corresponding to $\tilde{u}^{\mathrm{S}}=1.45 \mathrm{~km} / \mathrm{s}$. The high-speed case corresponds to an experiment (shot $2 \mathrm{~S} 47$ ) due to Gustavsen et al [21]. We shall find that the events leading up to the formation of a steady, ZND structure in each of the two cases are different. 


\subsection{The low-speed case}

We present the full evolution in stages. The first stage is shown in Figures 3, 4 and 5. Figure 3 provides a general impression of evolution from $t=0$ to $t=1.7$. During this entire period, the lead shock experiences only a marginal increase in its strength due to the chemical activity behind it. Energy liberated by the reaction generates a pressure pulse whose peak, at early times, is located at the wall, and later moves into the interior. The pulse amplifies, steepens, and in due course leads to the formation of a second shock behind the lead shock. A typical set of profiles at a time prior to the formation of the second shock is displayed in figure 4. Figure 4(a) shows the snapshots of $p, v$ and $\lambda$. We observe, from figure 4(b), that the minimum in $v$ and the maximum in $p$ occur at $\lambda=0.8$. This location is also the site of the sudden drop in reaction rate, figure 4(c), where the first, stronger growth term switches off in favor of the second, weaker growth term. The ignition step is barely noticeable at the scale of the figure.

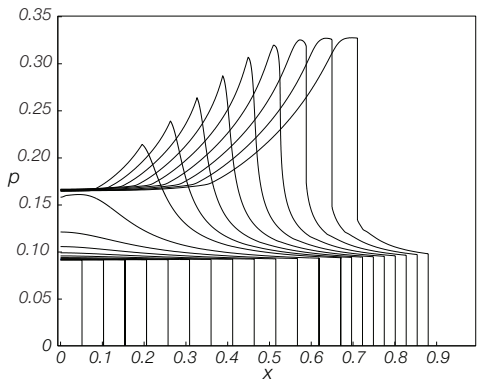

(a)

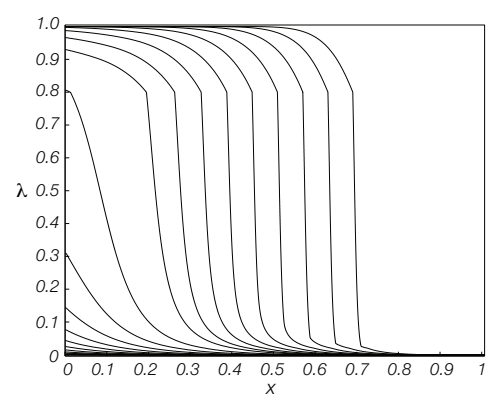

(b)

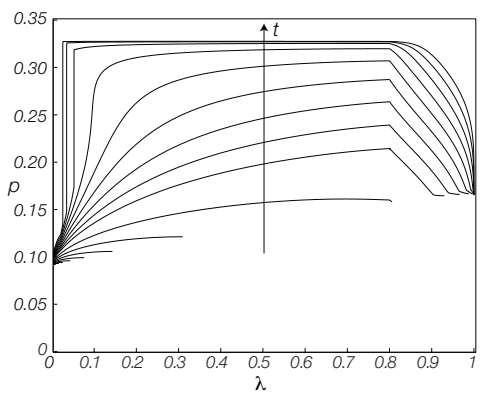

(c)

Figure 3: Stage $1, t=0$ to 1.3 by $0.1, t=1.3$ to 1.7 by 0.05 . Plots of (a) $p$ against $x$, (b) $\lambda$ against $x$, and (c) $p$ against $\lambda$.

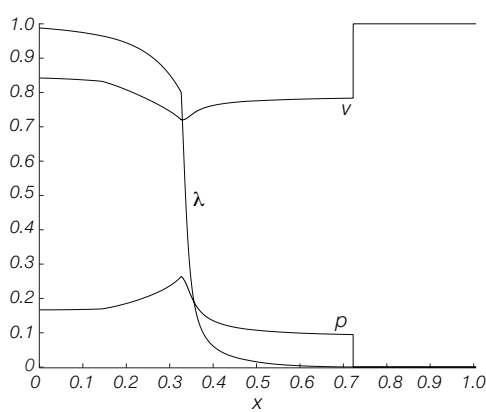

(a)

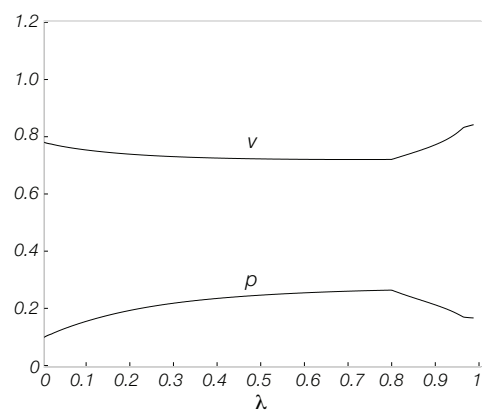

(b)

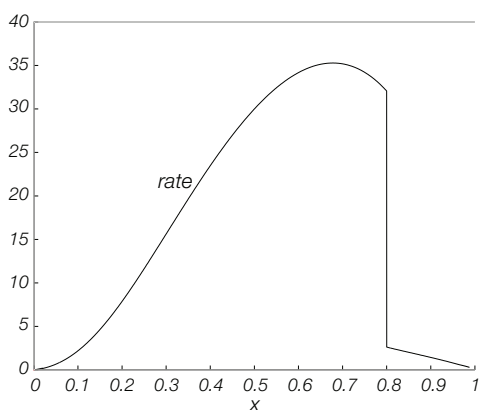

(c)

Figure 4: Stage 1, $t=1.4$. Plots of (a) $p, v$ and $\lambda$ against $x$, (b) $p$ and $v$ against $\lambda$, and (c) and reaction rate against $x$.

A typical set of profiles subsequent to the formation of the second shock are displayed in figure 5 . Figure 5(a) shows the snapshots of $p, v$ and $\lambda$. In figure 5(b) we see that the maximum pressure and minimum volume now occur essentially at the second shock. Ahead of this shock the reaction zone is compressive. Behind the shock, pressure and volume exhibit a long plateau followed by an expansion near the end of the reaction zone. The reason for the plateau can be understood by referring to the $p v$-plot of figure 5(c), in which the sharp peak corresponds to the state immediately behind the second shock. Because the evolution of this shock can be deemed to be quasisteady, the Hugoniot curves have a role to play. As the peak in figure 5(c) is close to the point of intersection of the Hugoniots, a bulk of the reaction takes place essentially without change of state, and the unsteady expansion is associated with only the tail of the reaction zone. 


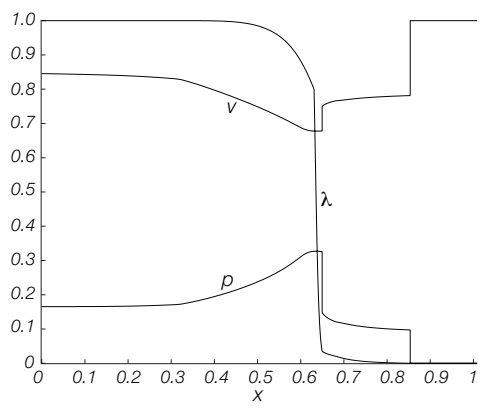

(a)

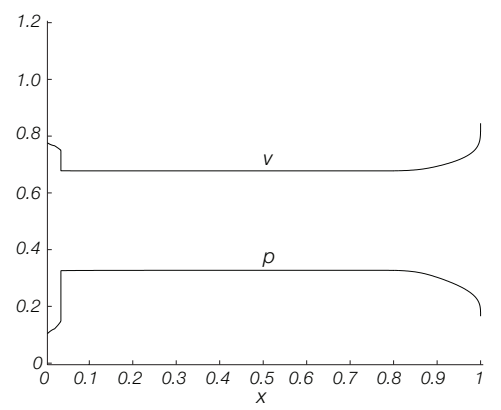

(b)

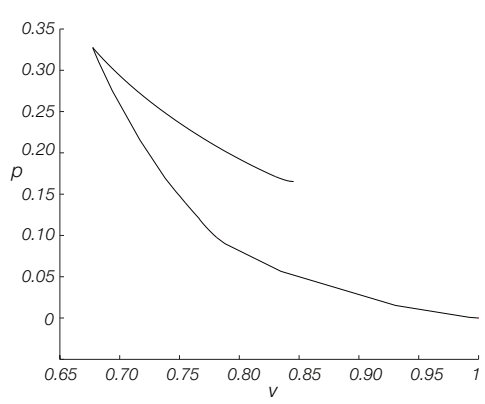

(c)

Figure 5: Stage 1, $t=1.65$. Plots of (a) $p, v$ and $\lambda$ against $x$, (b) $p$ against $\lambda$, and (c) $p$ against $v$.

As time progresses, the second shock approaches the lead shock. We pick up the second stage of evolution at $t=1.85$. The results, displayed in figure $6(\mathrm{a}, \mathrm{b})$ as profiles of $p$ and $v$, show that upon collision of the two shocks, a single shock propagates forward into the unreacted explosive while a contact and a rarefaction propagate backwards.

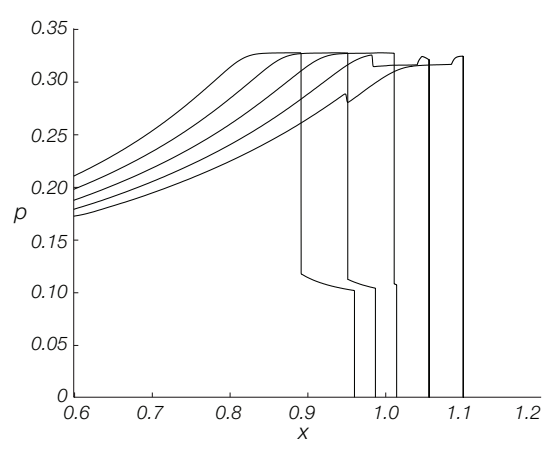

(a)

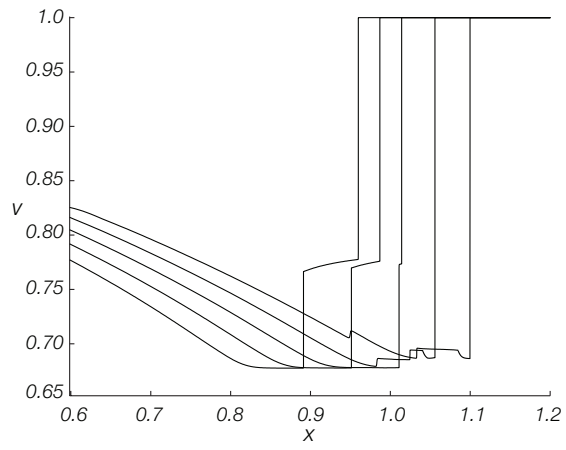

(b)

Figure 6: Stage 2, $t=1.85$ to 2.05 by 0.05 . Plots of (a) $p$ against $x$, and (b) $v$ against $x$.

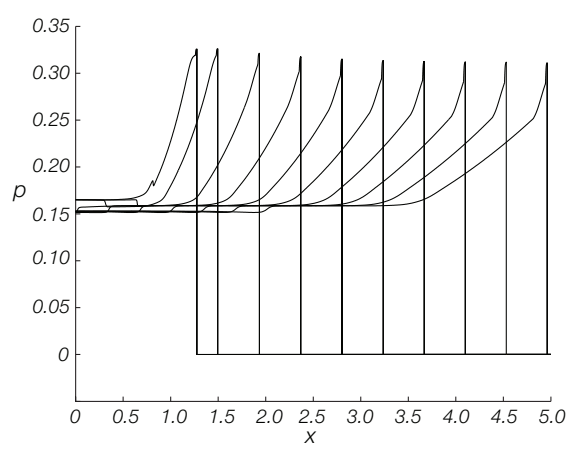

(a)

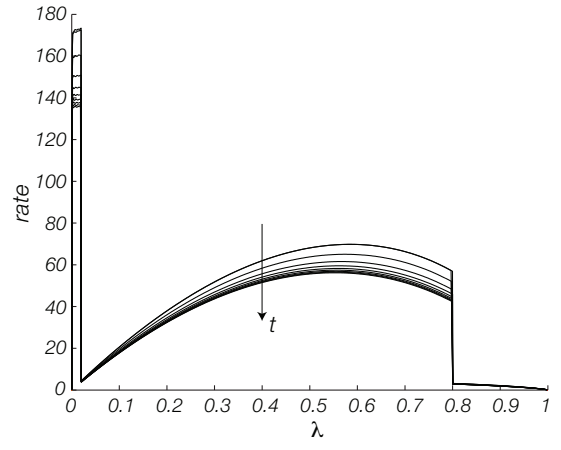

(b)

Figure 7: Stage $3, t=2.5$ to 6.5 by 0.5 . Plots of (a) $p$ against $x$, and (b) reaction rate against $\lambda$.

Stage 3 corresponds to the final evolution of the structure towards the steady, CJ-wave, and is shown in figure 7. Figure 7(a) shows the snapshots of pressure, while figure 7(b) plots those of reaction rate against reaction progress. We observe that the CJ state is approached gradually from above. 


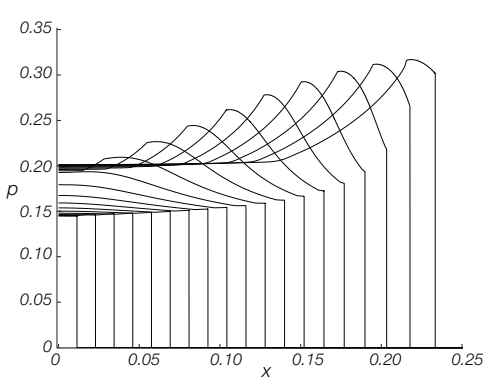

(a)

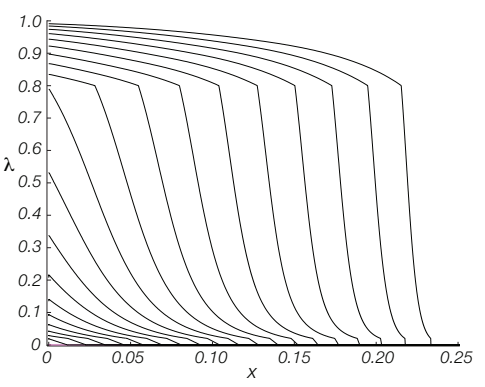

(b)

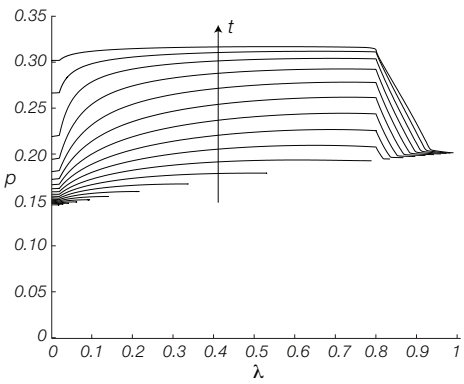

(c)

Figure 8: Stage $1, t=0$ to 0.38 by $0.02, t=1.3$ to 1.7 by 0.05 . Plots of (a) $p$ against $x$, (b) $\lambda$ against $x$, and (c) $p$ against $\lambda$.

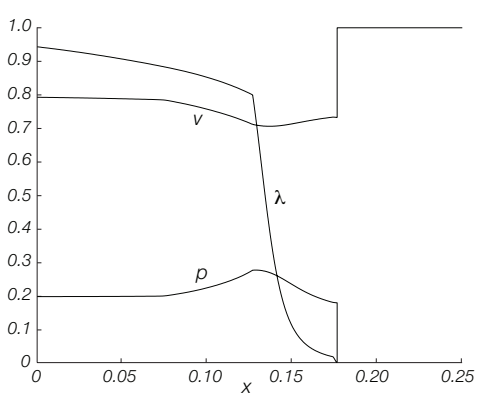

(a)

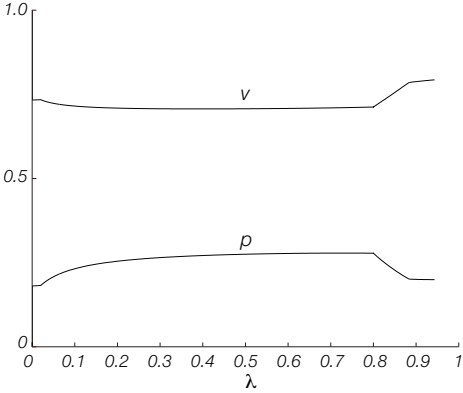

(b)

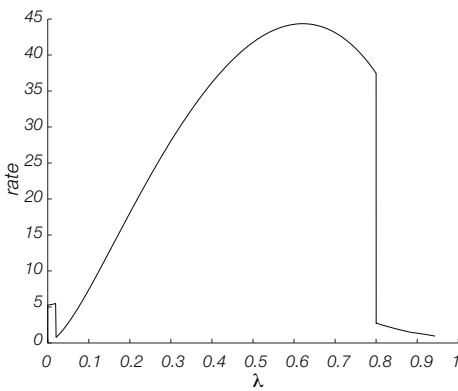

(c)

Figure 9: Stage 1, $t=0.3$. Plots of (a) $p, v$ and $\lambda$ against $x$, (b) $p$ against $\lambda$, and (c) and reaction rate against $x$.

\subsection{The high-speed case}

In this case two stages suffice to describe the evolution. The general features of the first stage, lasting from $t=0$ to $t=0.38$, are displayed in figure 8 . The reaction behind the shock generates a pressure pulse moving away from the boundary and undergoing amplification. Unlike the low-speed case, the pulse does not steepen into a secondary shock. Instead, it is closely coupled to the lead shock and strengthens it, first gradually and then with increasing rapidity. A typical set of stage- 1 snapshots at a specific time is displayed in figure 9. Figure 9(a) shows the profiles of $p, v$ and $\lambda$. Figure 9(b) shows that the minimum in $v$ and the maximum in $p$ occur at $\lambda=0.8$, just as they did for the low-speed case. The reaction rate, figure 9(c), has a similar behavior, with the exception that the ignition step is of significant size, and is in fact more prominent than the second growth term.

Stage 2 corresponds to the final evolution of the structure towards the steady, CJ-wave, and is shown in figure 10. Figure 10(a) shows the snapshots of pressure, while figure 10(b) plots those of reaction rate against reaction progress. We observe that the evolution is similar to that for the low-speed case, only faster.

For this case we also show the evolution of particle velocity at a set of ten Lagrangian marker particles. This situation mimics the particle-gauge experiments of Gustavsen et al [21], with whose experimental observations these computational results can be compared, see figure 11. We observe, as expected, that the agreement is not strong, since the parameter set adopted here is not the 'initiation set.' Gustavsen et al [21] do provide the alternate data set and state that the measured and computed profiles are in good agreement.

To summarize, shock-initiation follows one of two scenarios. At low impact speeds the reaction generates a secondary shock behind the lead shock, the two shocks collide, and following a transient the detonation approaches the CJ wave. At high impact speeds there is no secondary shock; the reaction strengthens the lead shock itself to form the CJ wave. 


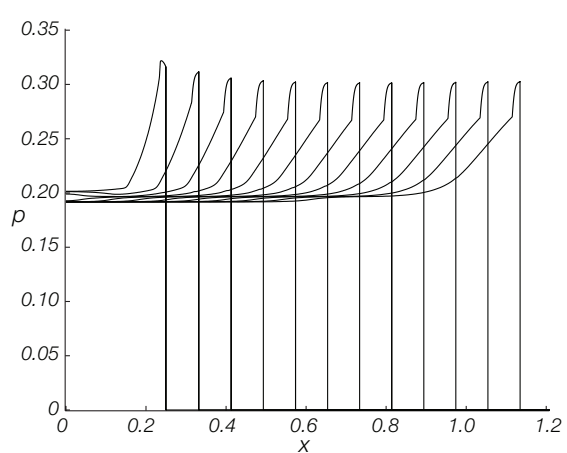

(a)

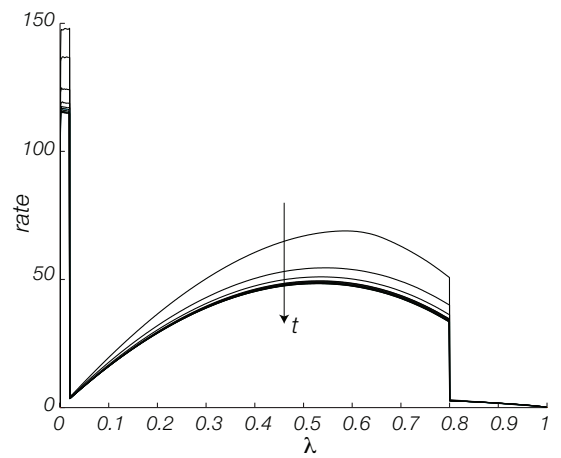

(b)

Figure 10: Stage 2, $t=0.4$ to 1.5 by 0.1 . Plots of (a) $p$ against $x$, and (b) reaction rate against $\lambda$.
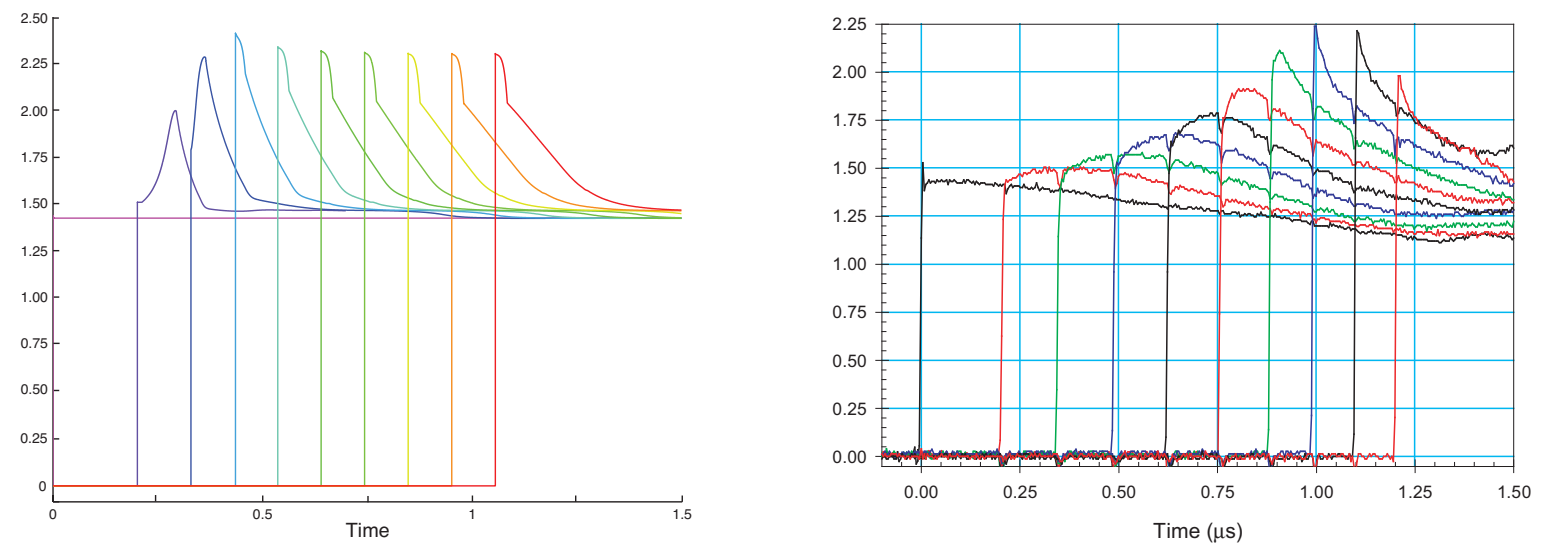

Figure 11: Velocity $(\mathrm{mm} / \mu \mathrm{s})$ histories at marker particles: computed (left) and observed (right).

\section{Planar Diffraction Around a $90^{\circ}$ Corner}

We now present results of 2D detonation diffraction as an established, steady, planar, CJ detonation propagating in a block of quiescent explosive rounds a $90^{\circ}$ corner. The geometry consists of a narrow channel two units wide and one and a half units long, abruptly expanding into a channel ten units wide and five units long. Figure 12 shows the lower half of the symmetric configuration. A steady CJ detonation is assumed to propagate through the narrow segment, and at the initial time the lead shock of this CJ wave is located at a position one half unit from the inlet. The speed of the wave is unity and the particle velocity at the end of the reaction zone has the dimensionless CJ value 0.2403 , which is also set as the inlet boundary condition for all time. Initially, the region between the inlet and the end of the reaction zone is taken to be at the CJ state. The walls of the channel are held rigid, and a no-reflection boundary condition is applied at the outlet. In order to avoid the corner singularity, the sharp corner is rounded with a radius of approximately one-half of the length of the steady reaction zone.

The early-time results, from $t=1$ to $t=1.4$, are shown in figure 13. A triptych is shown at each time level, displaying from left to right the contours of pressure, the contours of $\lambda$, and a numerically generated schlieren plot that highlights density gradients, thereby serving to identify shocks and contact lines.

At $t=0$ the planar detonation, poised at a position one length unit upstream of the corner, begins its travel through the narrow channel as a steady, undisturbed wave. It first senses a change in geometry upon arriving at the corner, at approximately $t=1$, see figure 13(a). As the wave rounds the corner, the expansion generated there is felt by the lead shock, first at the wall and then in a continually expanding 


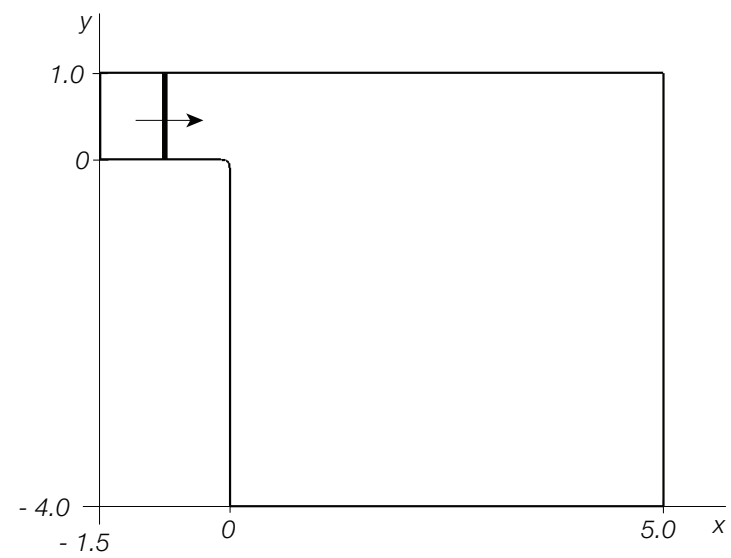

Figure 12: Computational configuration for diffraction around a $90^{\circ}$ corner.

domain centered at the corner. This causes the shock in the near-corner region to weaken, acquire curvature, and suffer a deceleration, as it would in a nonreacting medium. Here the additional feature is a commensurate drop in the rate of reaction behind the shock, which causes a progressive retreat, most conspicuously at the wall, of the reaction zone following the shock. Near the line of symmetry (top boundary of the computational domain) the detonation stays planar, and at these early times continues to propagate virtually undisturbed into the expanded channel. The plots of pressure $p$ in figures 13 (b) and (c), at $t=1.2$ and 1.4 respectively, show the spreading expansion and the associated weakening and curvature of the lead shock. The plots of the reaction progress variable $\lambda$ in the same figures show the reaction zone falling behind the shock. Both of these features are captured well by the schlieren plots in these figures.

Further insight into the near-wall behavior of the wave can be gained by examining the profiles of density $\rho$ and progress $\lambda$ at the wall, shown in figure 14. The first pair of profiles at $t=0.8$ corresponds to the undisturbed planar wave that has yet not reached the corner. The second pair at $t=1.0$ shows that the presence of the corner has just been felt, as evidenced by the small reduction in density behind the shock. At $t=1.2$ the shock has weakened further, and the reaction zone has retreated significantly from the shock. A sharp interface has developed between the denser unreacted material and the lighter products of reaction; it can be seen both in the sharpened $\lambda$ profile and in the well-defined density jump. This feature persists at $t=1.4$, and the interface lags even farther behind the lead shock. At least for the time being the detonation has failed near the wall.

A word about this sharp drop in the reaction rate behind the shock is in order. Recall, from equation (10) for the rate law, that for the ignition term in the rate to be switched on, density $\rho$ must exceed a threshold, i.e., $\rho>1+a=1.22$, since $a=0.22$ from Table 2 for the explosive under consideration. Figure 14 displays the density level 1.22, and shows that at $t=1.4$ for example, density behind the lead shock is only slightly above the threshold. Since the ignition term in (10) is proportional to $(\rho-1-a)^{x}$, and $x=7$ from Table 2, the very slight density excess above the threshold leads to a positive but extremely weak contribution from the ignition step. It is also worth noting, in figure 14, that in the product region behind the reactant/product interface, corner expansion has produced very small density values. However, these have no effect on the evolutionary process as the density minima occur in the fully reacted region a substantial distance behind the lead shock.

Returning to figure 13(c), we note the appearance of another feature in the pressure plot at $t=1.4$, namely, a thin ridge of high pressure developing behind the shock. (A hint of the ridge can already be seen at $t=1.2$ in the pressure plot of figure 13(b).) The ridge is closest to the shock at a point about half way down from the top boundary, but is increasingly withdrawn from it as one travels farther down along the shock. The pressure and schlieren plots in figure 15(a) reveal the ridge to be both elongated and strengthened at $t=1.6$. At $t=1.8$, in the pressure plot of figure 15(b), the upper segment of the ridge 

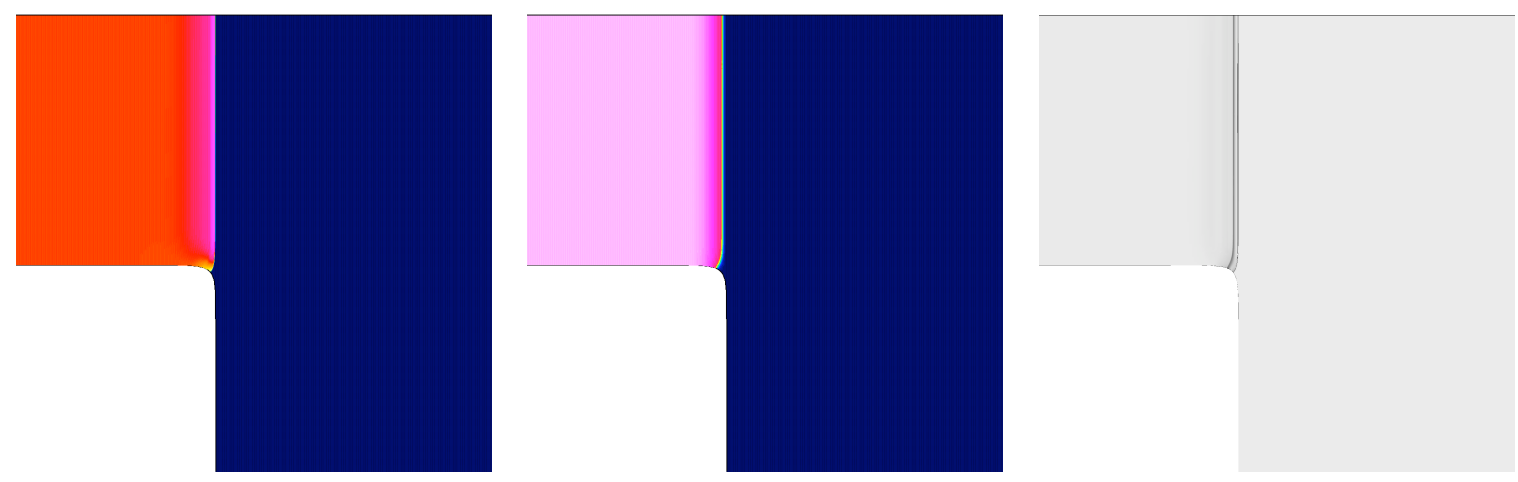

(a) $t=1.0$
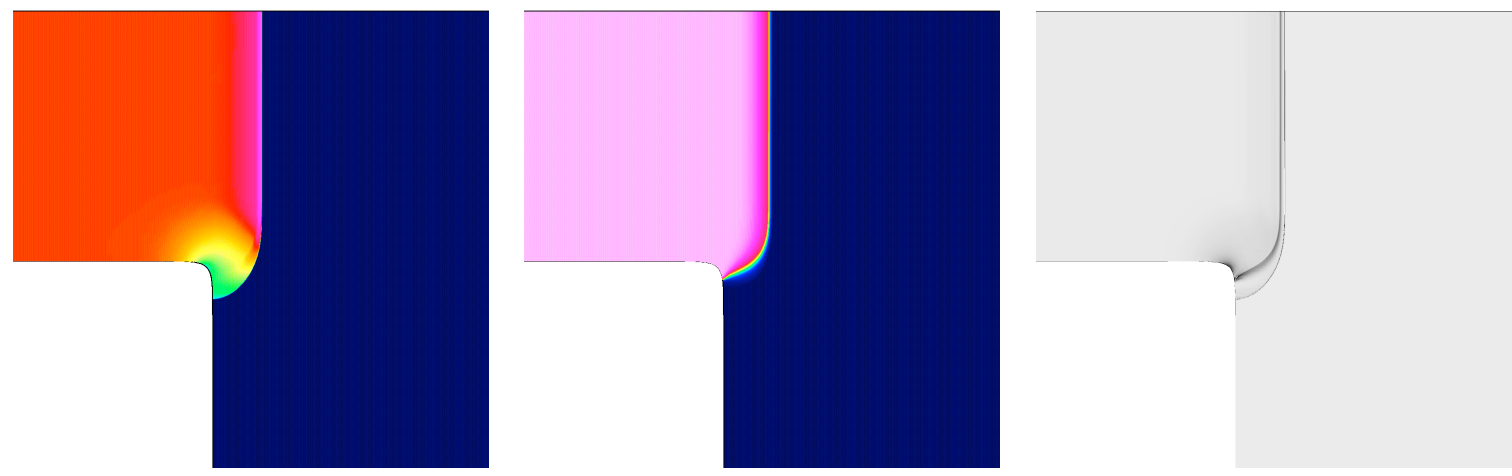

(b) $t=1.2$
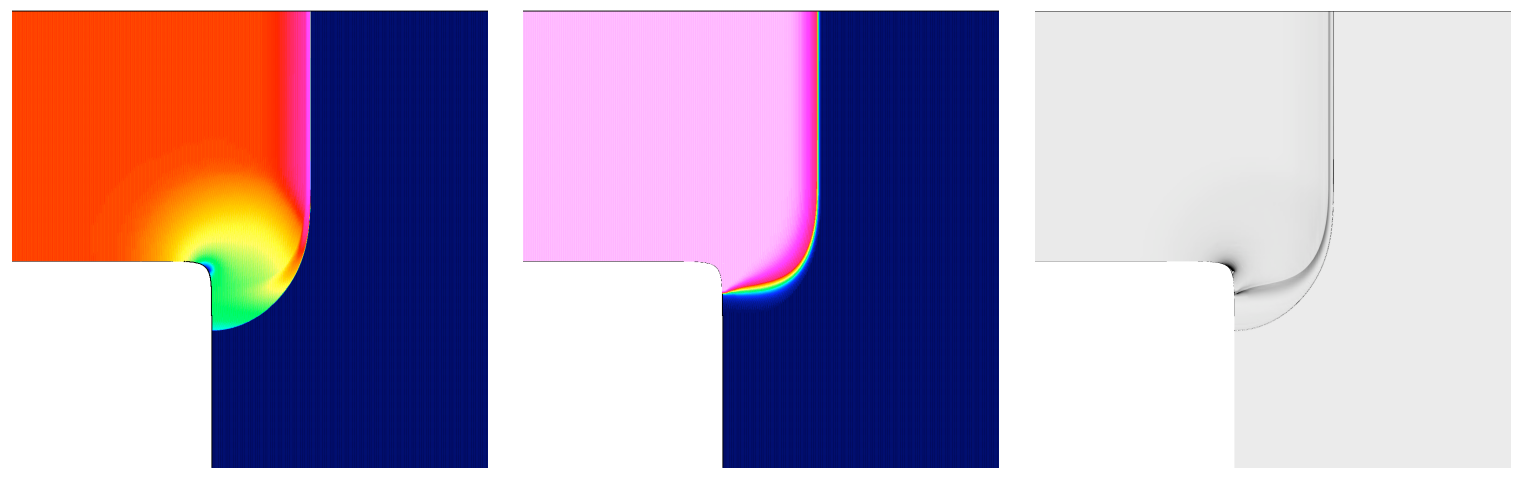

(c) $t=1.4$
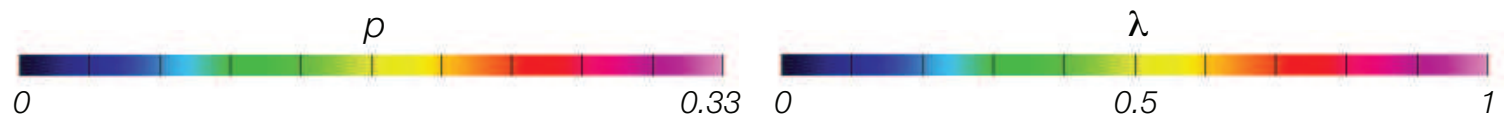

Figure 13: Shaded contours of $p$ (left), $\lambda$ (middle) and numerically-generated Schlieren (right) at three early times. The color bars for $p$ and $\lambda$ are also shown. 


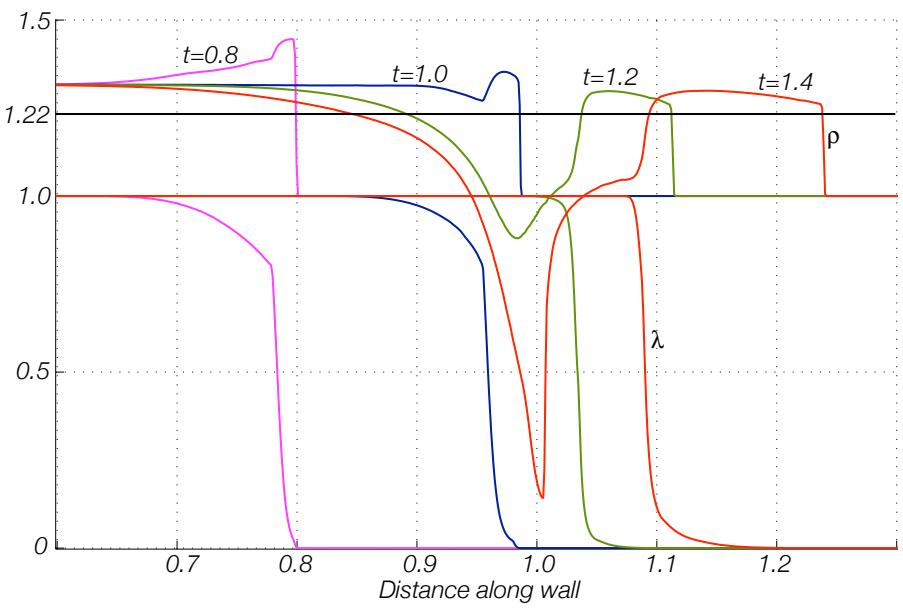

Figure 14: Profiles of density and reaction progress at the wall, $t=0.8$ to 1.4 by 0.2 .

is seen to merge with the lead shock and strengthen it, while the lower segment connects up with a similar but weaker ridge that has just developed in the region adjacent to the wall, a significant distance behind the lead shock. The $\lambda$ plot of figure 15(b) shows the near-wall segment of the ridge to also be a site of enhanced chemical activity. At $t=1.9$, the pressure and schlieren plots of $15(\mathrm{c})$ show that a secondary shock has appeared at the leading edge of the ridge near the wall, while the $\lambda$ plot shows a strengthened and narrowed reaction zone immediately behind the secondary shock. Thus a fledgling detonation has appeared in what had temporarily been a dead zone next to the wall, and this detonation is on its way to colliding with the weak lead shock; see figure 16 corresponding to $t=2.0$.

The mechanism for the rebirth of the detonation can be understood by referring to figure 17 . This figure continues the display of wall values of $\lambda$ and $\rho$ begun in figure 14. The first pair of profiles, corresponding to $t=1.4$, is carried over from figure 14 and shows a weak shock followed by the nearly dormant reaction, with a sharp interface separating reactant and product. The next pair at $t=1.6$ shows the first stirrings of renewed chemical activity; the effect is even more pronounced in the profiles at $t=1.8$. One now sees a wellestablished reaction zone, with only a vestige of the erstwhile density jump near its tail, and with a peak in density created by the energy released in the reaction. This density peak is the 'along-the-wall' manifestation of the pressure ridge seen earlier in figure 13(c). At $t=2.0$ the peak has amplified and steepened into a secondary shock; the reaction zone has come to resemble that of a planar detonation. Collision between the primary and secondary shocks is imminent; also see figure 16. Subsequent evolution to a virtually steady ZND structure is evident in figure 18, which carries the wall profiles of $\rho$ and $\lambda$ further in time to $t=3.6$.

It is instructive to compare the evolution along the wall as displayed in figures 14, 17 and 18 to the planar, 1D, low-speed shock-initiation displays of figure 4. We note that the principal features of figure 4: (i) the birth of a pressure pulse behind the lead shock, (ii) the steepening of the pulse into a secondary shock, and (iii) the subsequent collision of the secondary shock with the lead shock on the way to a ZND wave, are mimicked in the wall profiles just discussed. This clarifies the mechanism responsible for the reappearance of detonation; the weakening of the lead shock upon diffraction around the corner, and the consequent near-failure of the reaction behind it, set up conditions much like those encountered in planar initiation by a low-speed shock. It takes some time for the reaction behind the lead shock to intensify, but once it does, it leads to a local explosion some distance behind the shock.

Three additional observations complete the description. The first refers to figure 19 which displays two schlieren plots of the region near the corner, at $t=2.0$ and $t=2.6$. These views show the complex vortex-shock structure at the corner, continuing to evolve long after the lead shock has gone by. For the second we turn to figure 20 which shows the profiles of $\rho$ from $t=1$ to $t=3.6$, along the wall and along the line of symmetry. We see that on the line of symmetry the reaction-zone profile is only mildly disturbed, 

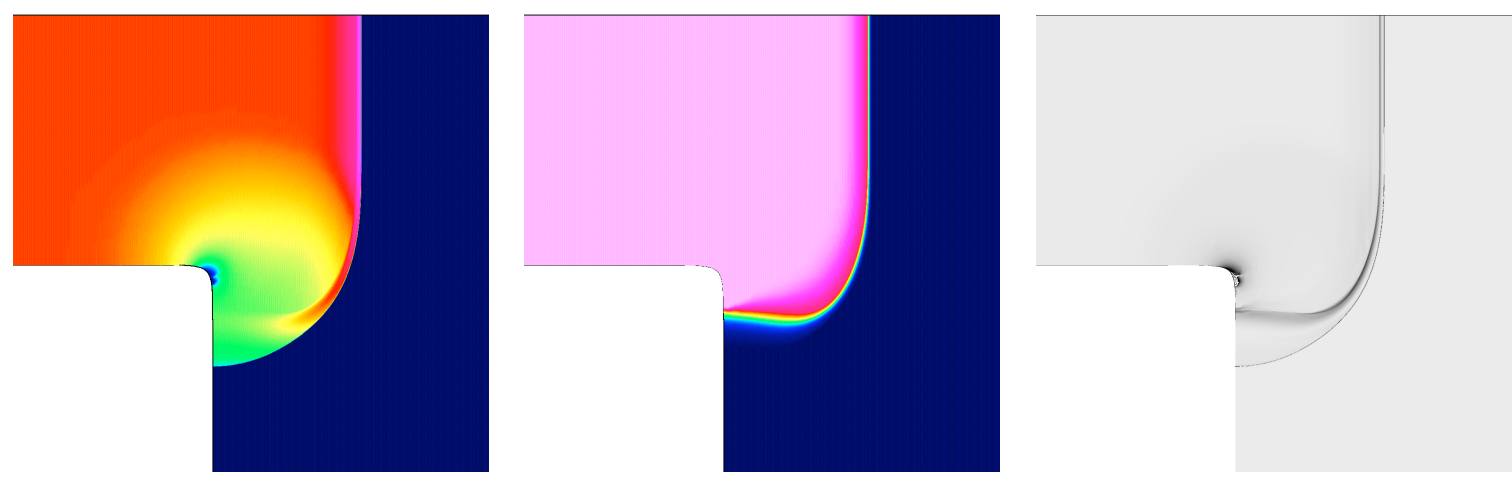

(a) $t=1.0$
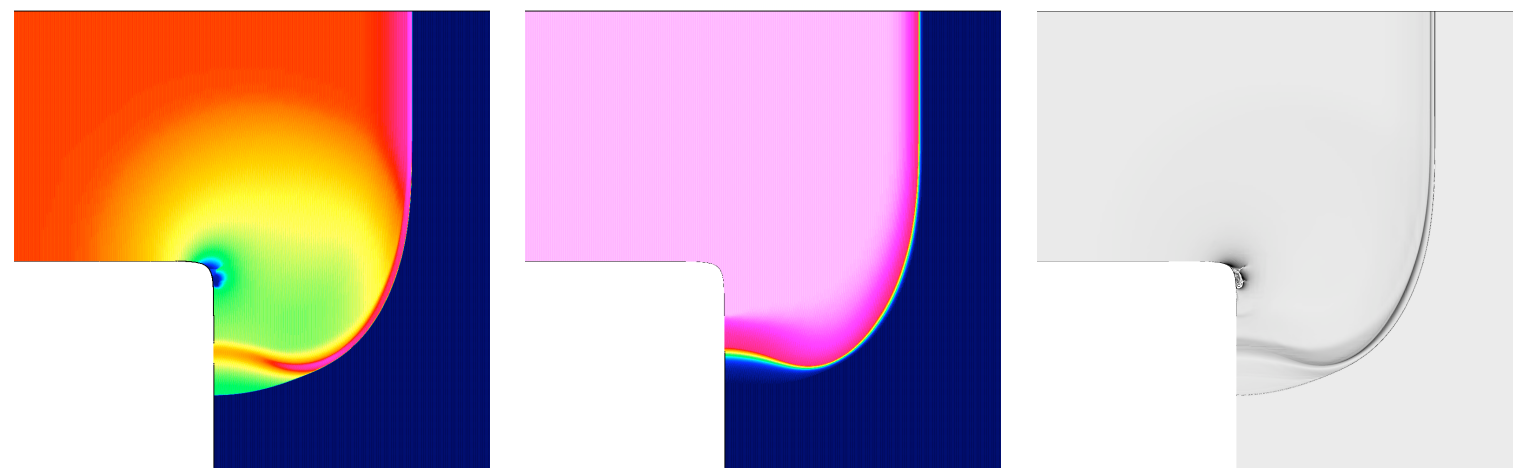

(b) $t=1.2$
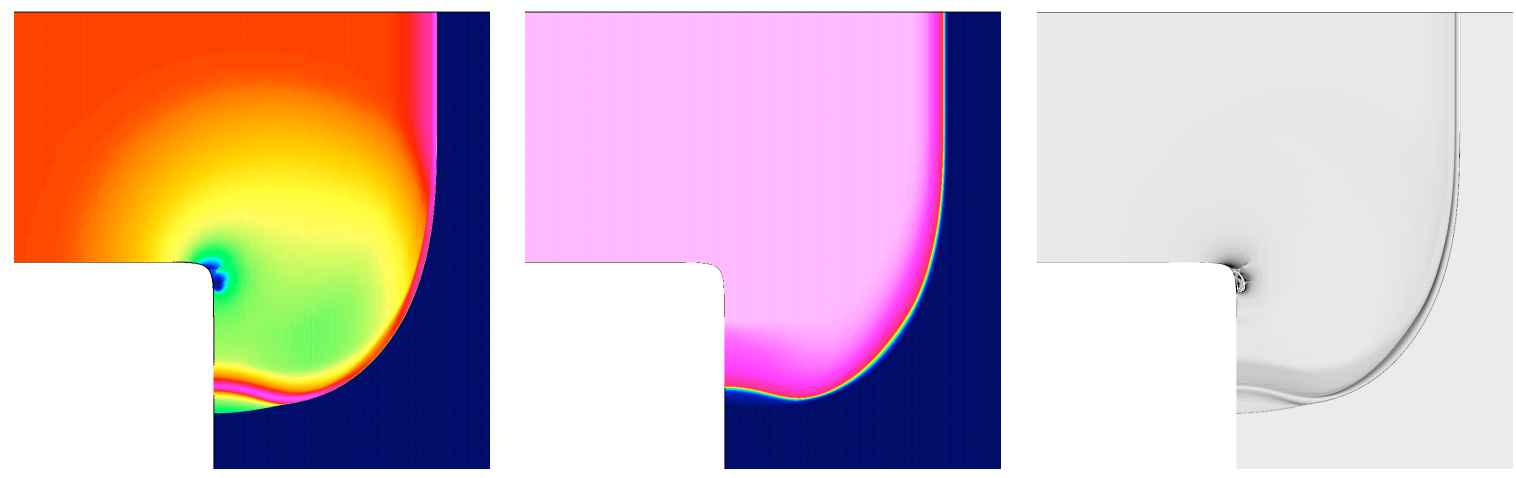

(c) $t=1.4$

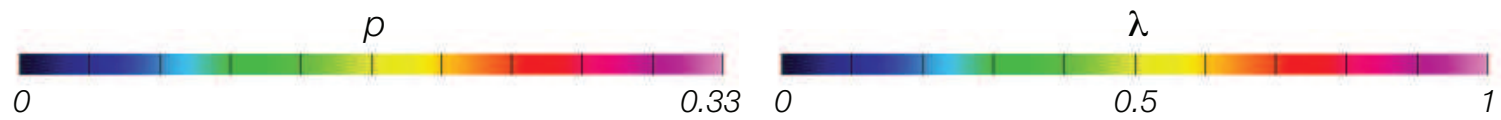

Figure 15: Shaded contours of $p$ (left), $\lambda$ (middle) and numerically-generated Schlieren (right) at three middle times. 
while the trailing expansion region shows some effects of the signals of expansion emanating from the corner. By contrast, the evolution along the wall is more complex, as discussed in detail above. Third, subsequent to the diffraction of the lead shock past the corner, pressure and density in the fully reacted region at the corner can sink to rather low values, with density falling substantially below the ambient value as shown in figure 21. We proceed under the assumption that the equation of state continues to hold in this region of extreme expansion. The sound speed remains real and no numerical difficulty is encountered.

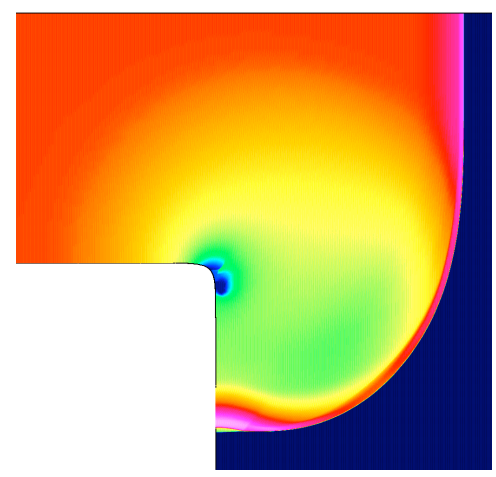

$p$
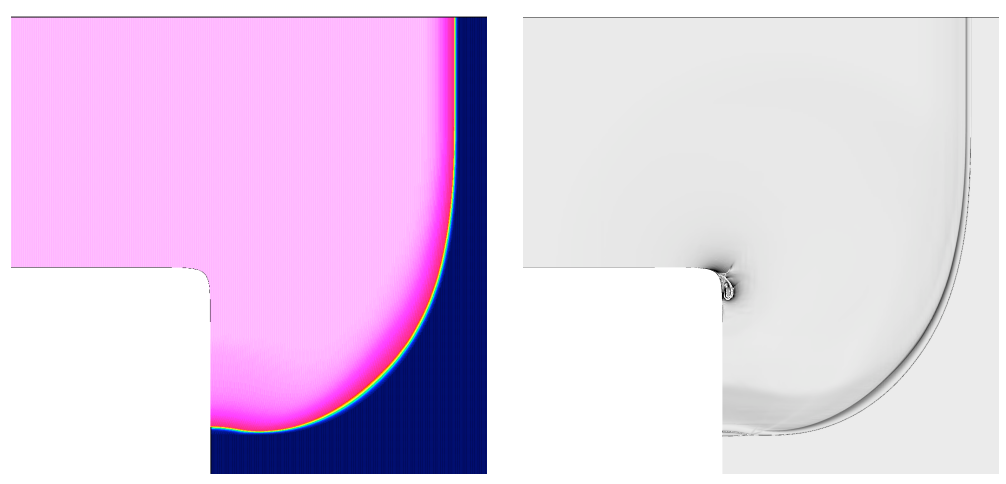

$\lambda$

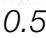

Figure 16: Shaded contours of pressure (left), $\lambda$ (middle) and numerically-generated schlieren (right) at $t=2.0$.

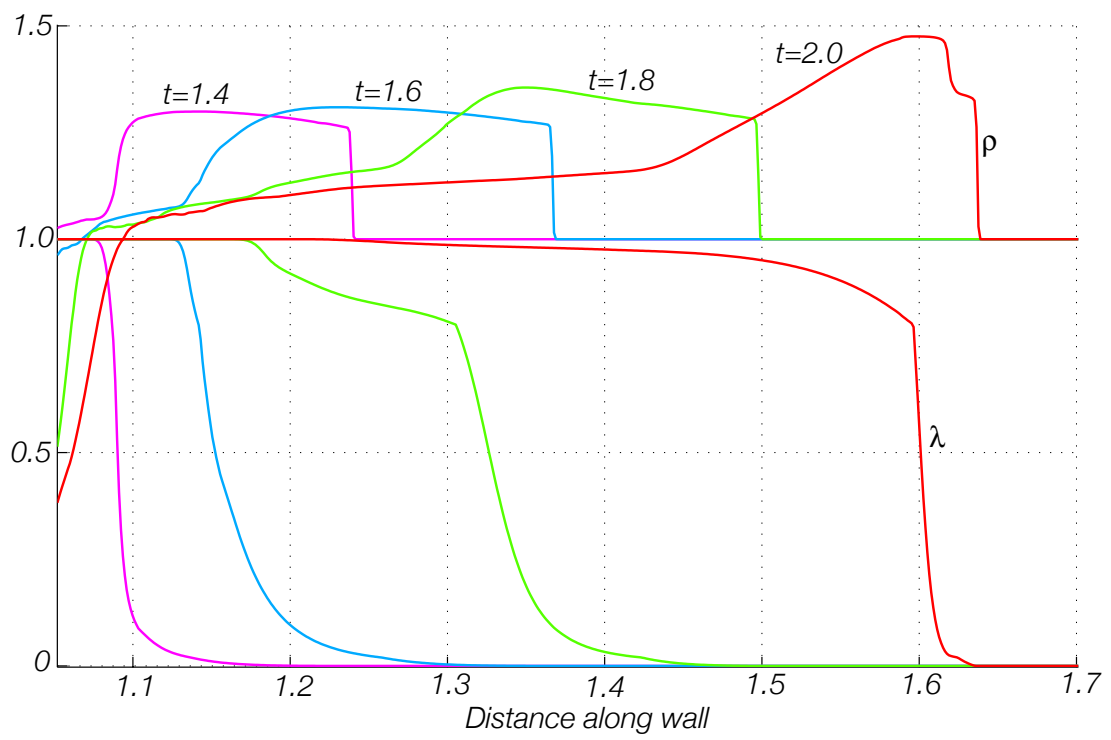

Figure 17: Profiles of density and reaction progress at the wall, $t=1.4$ to 2.0 by 0.2 . 


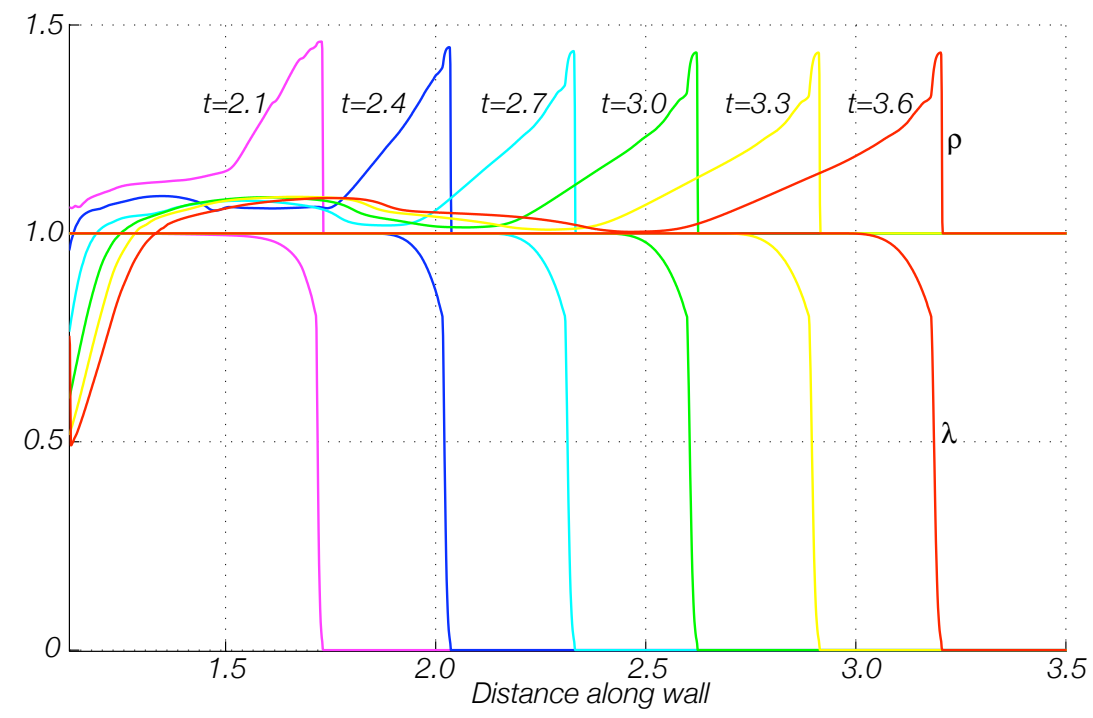

Figure 18: Profiles of density and reaction progress at the wall, $t=2.1$ to 3.6 by 0.3 .
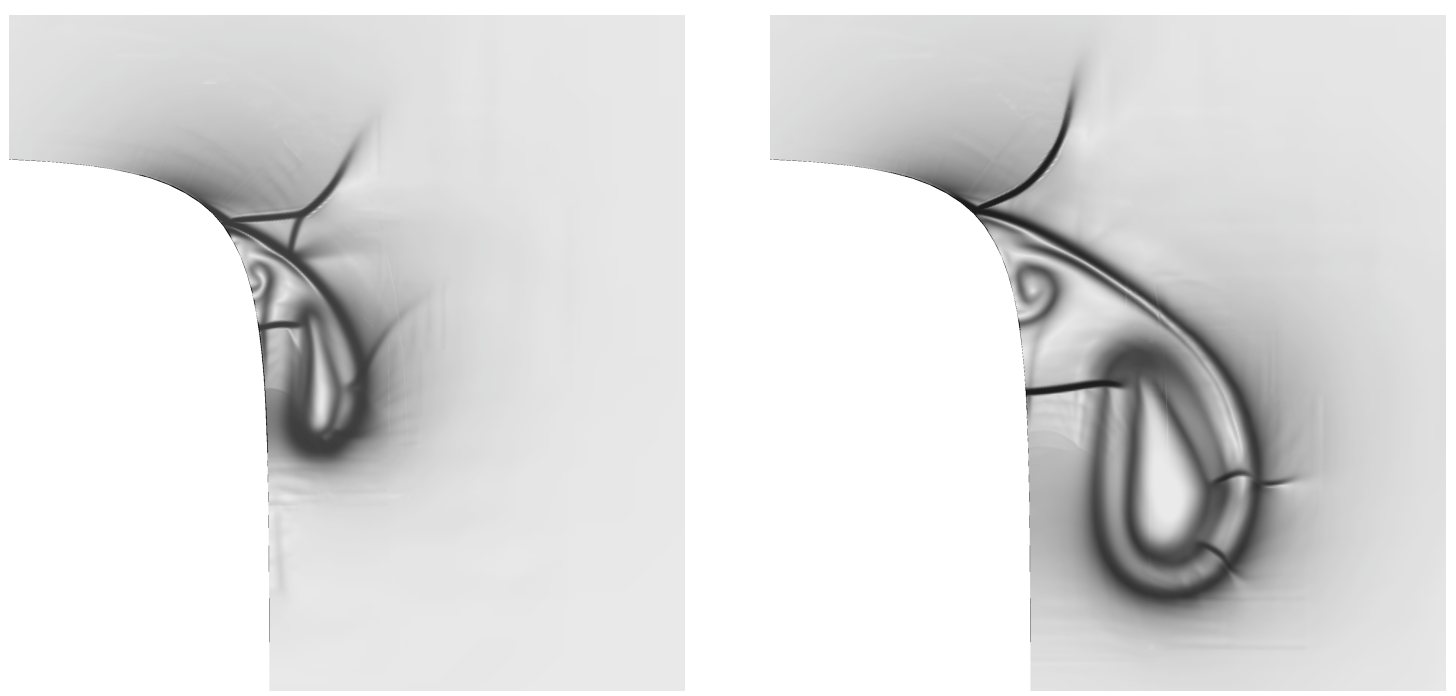

Figure 19: Schlieren plots in the near-corner region, at $t=2.0$ (left) and $t=2.6$ (right). 


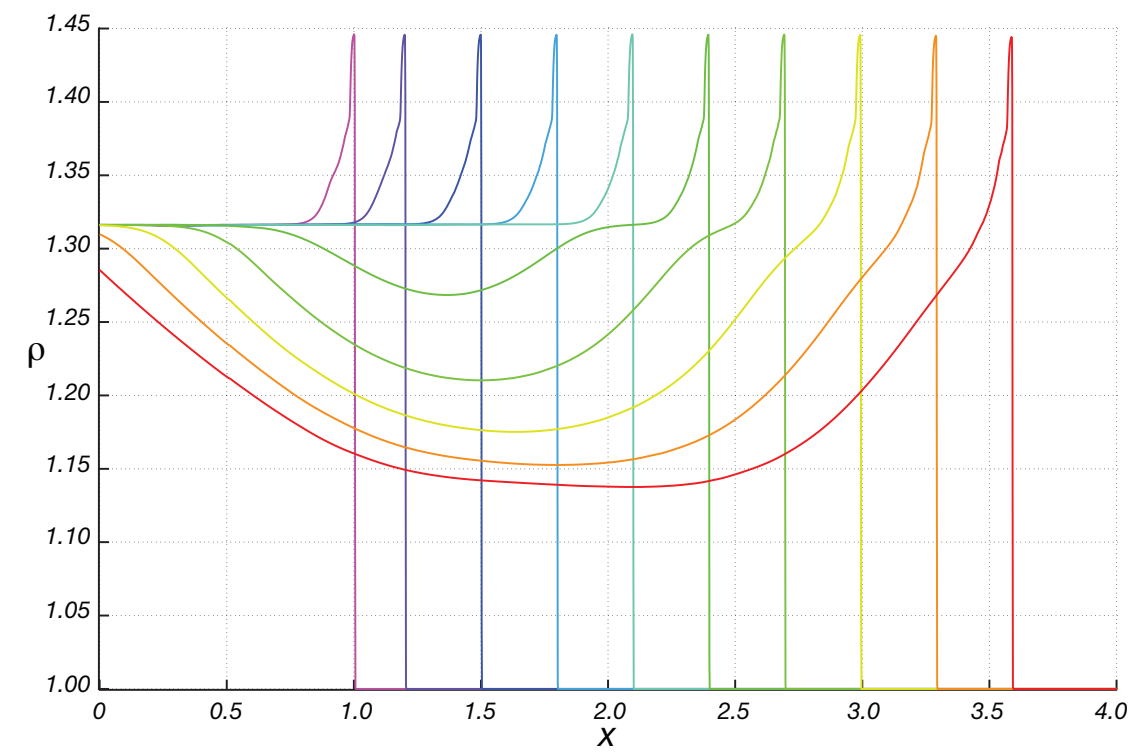

(a)

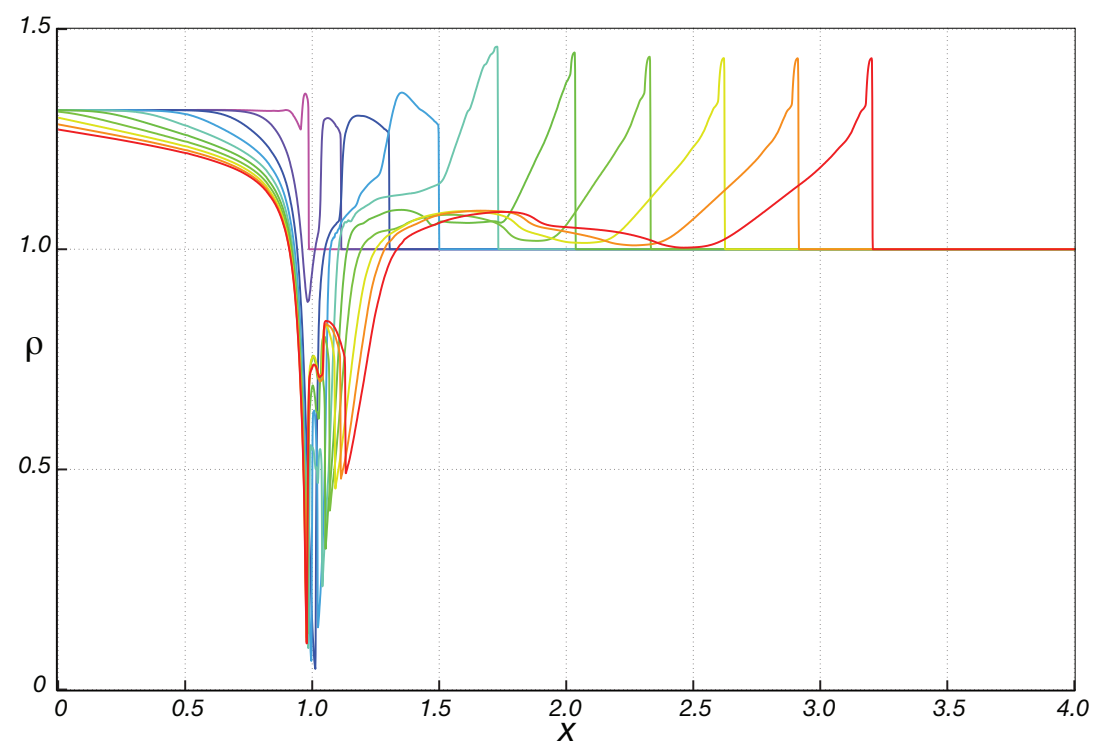

(b)

Figure 20: Profiles of density from $t=1.0$ to $t=3.6$ along the line of symmetry (a) and along the wall (b). 


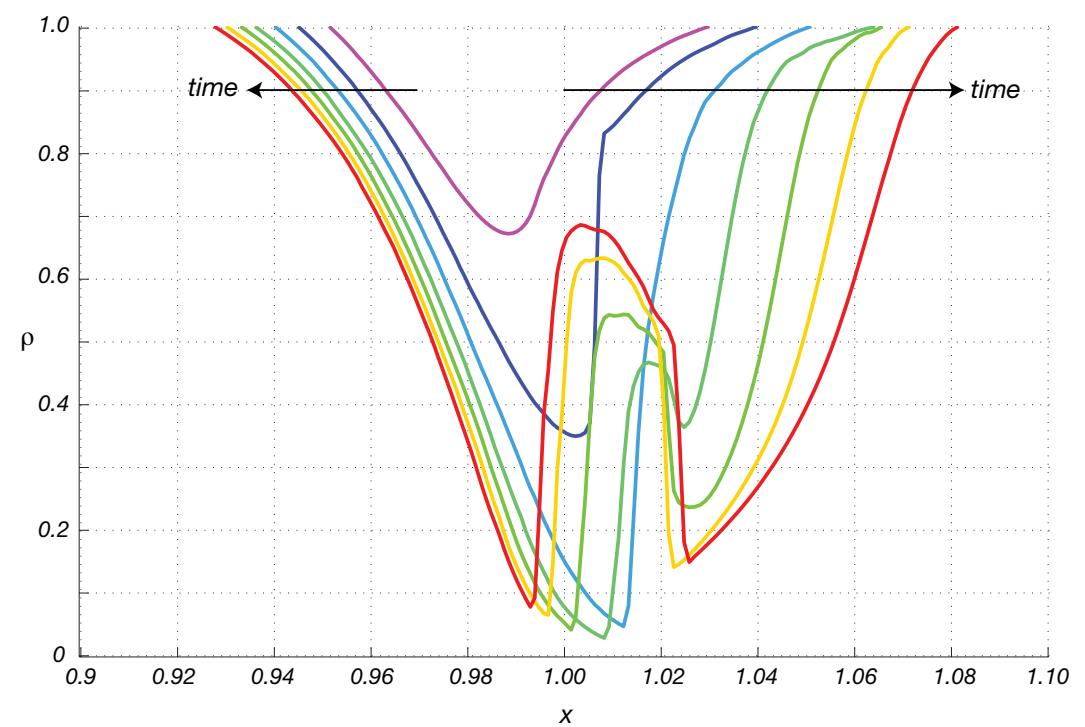

(a)

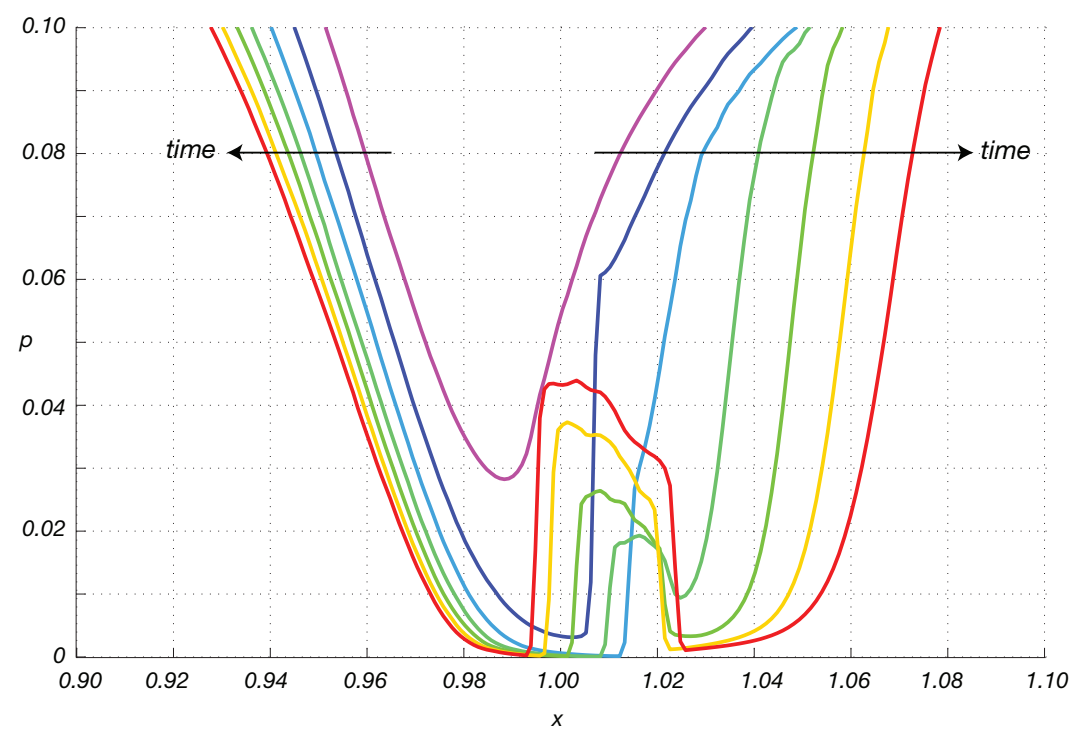

Figure 21: Profiles of density (a) and pressure (b) along the wall, from $t=1.3$ to $t=1.9$ by 0.1 . 


\section{Planar Diffraction Around a $140^{\circ}$ Corner}

In the case just examined, it was found that upon diffraction past a right-angled corner the reaction behind the lead shock was significantly weakened, but not extinguished, in the near-wall region. Consequently, the local decoupling of the reaction zone from the shock was short-lived, and the detonation managed to re-establish itself after a short run down the wall. In this section we consider a larger angle of diffraction to see whether the stronger expansion resulting therefrom is enough to extinguish the ignition step, and if so, whether the accompanying decoupling of the shock and the reaction survives to create a zone that remains permanently dead.

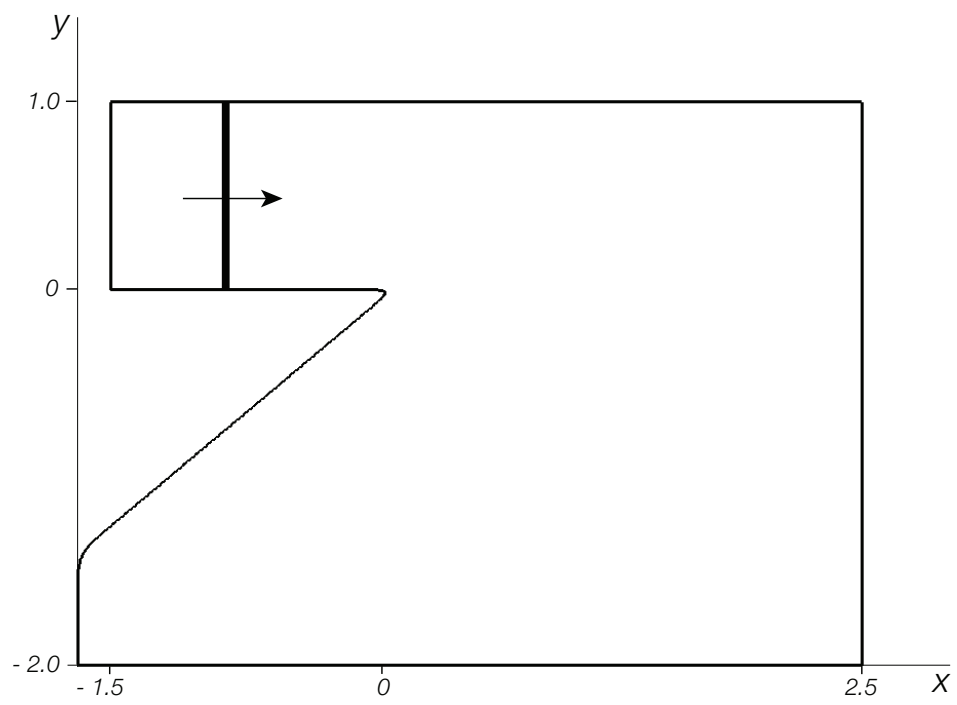

Figure 22: Computational configuration for diffraction around a $140^{\circ}$ corner.

The overall picture is seen in the pressure, $\lambda$ and schlieren plots of figures 23 and 24 . As before, the story begins at $t=1$, figure 23(a), when the steady, planar, CJ wave has just reached the end of the narrow channel. As the shock diffracts at the corner, the expansion weakens the coupling between shock and reaction, causing an increasingly broad separation between the shock and the edge of the fully reacted region, figures $23(\mathrm{~b})(t=1.4)$ and $23(\mathrm{c})(t=1.8)$. Diffraction through a larger angle also generates a stronger corner vortex than was seen at correspondingly early times for the right-angled corner.

A pressure ridge appears behind the lead shock, prominently seen in figure 23(c) $(t=1.8)$, curling away from the lead shock about $60 \%$ of the way down as one advances along the shock from the line of symmetry towards the wall. The portion of the ridge adjacent to the lead shock strengthens the lead shock, see figures 24 (a) to (c) corresponding to $t=2.0$ to $t=2.4$, while the portion curled away from the lead shock steepens into a secondary shock of its own. There is clear evidence in the $p, \lambda$ and schlieren plots of these figures that each of the shocks is tightly coupled to the reaction behind it. Essentially one has a curved detonation, a part of which propagates forward into the pristine material with the primary shock as the lead shock, and the other propagates transversely into the unreacted material (processed earlier by the weakened primary shock) with a secondary shock as the lead shock. A triple point at the intersection of the three shocks (primary, secondary and weakened primary) can clearly be seen in the pressure and schlieren plots of figure $24(\mathrm{a}-\mathrm{c})$.

At $t=2.5$ the plots of $p$ and $\lambda$ are shown in the the expanded views of figure 25(a). These plots show that the transverse wave has nearly reached the wall. The $\lambda$ plot shows two other features. First, the corner vortex has entrained a small quantity of unreacted material within it. Second, a narrow well of reactive material appears between two fully reacted regions: the near-corner product region at a low pressure, and 

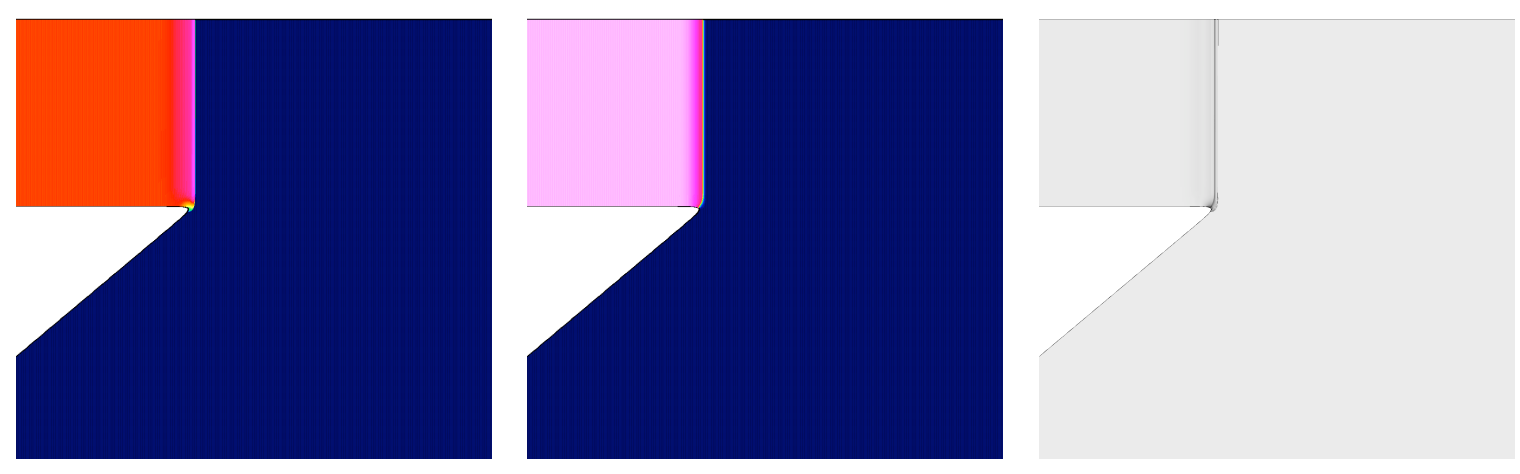

(a) $t=1.0$
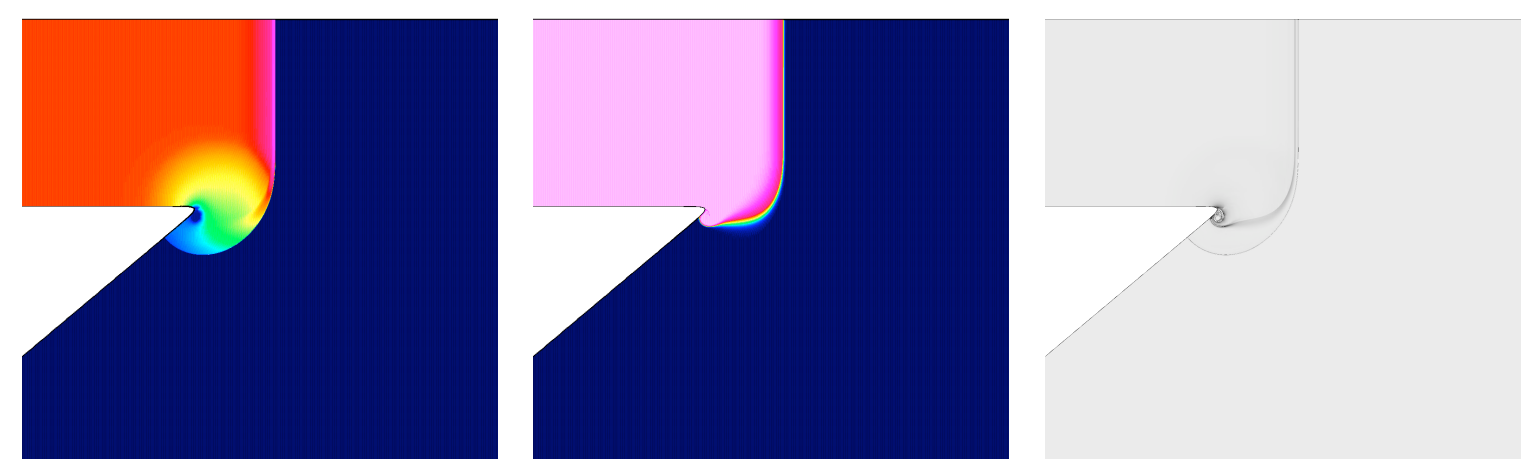

(b) $t=1.4$
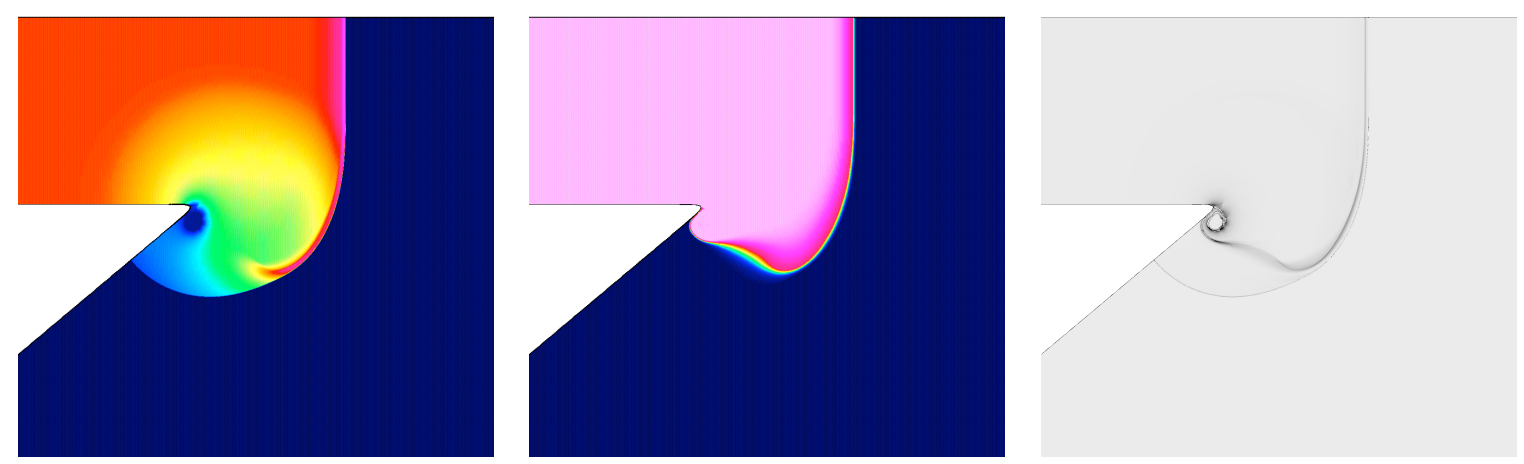

(c) $t=1.8$

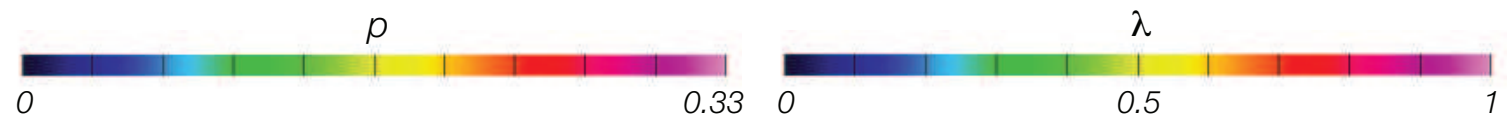

Figure 23: Shaded contours of $p$ (left), $\lambda$ (middle) and numerically-generated schlieren (right) at $t=$ 1.0, 1.4 and 1.8. 

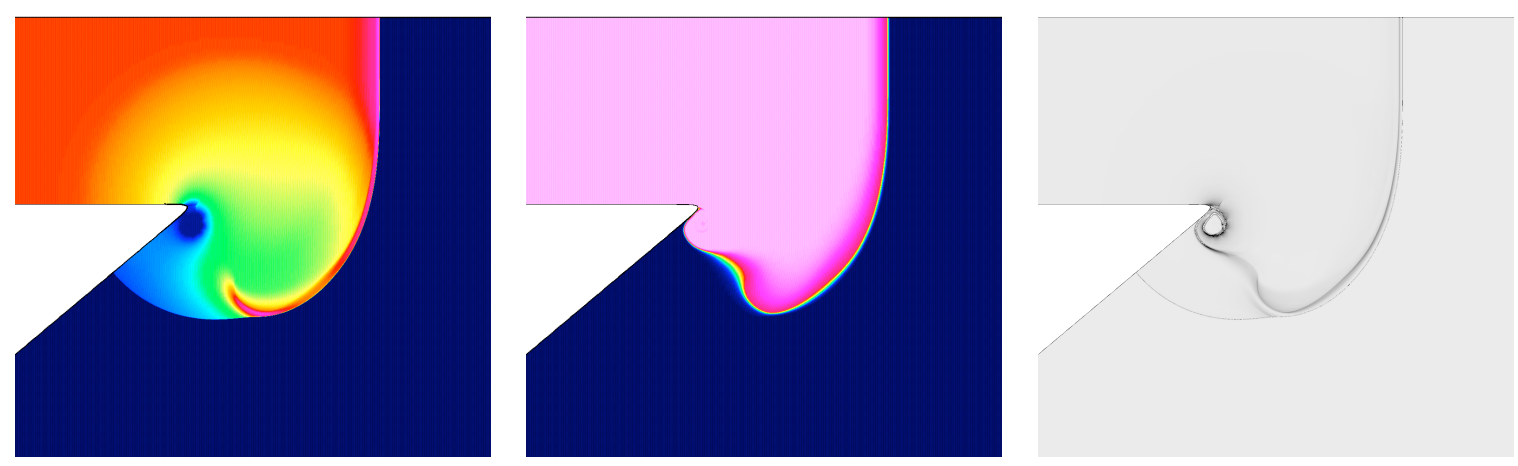

(a) $t=2.0$
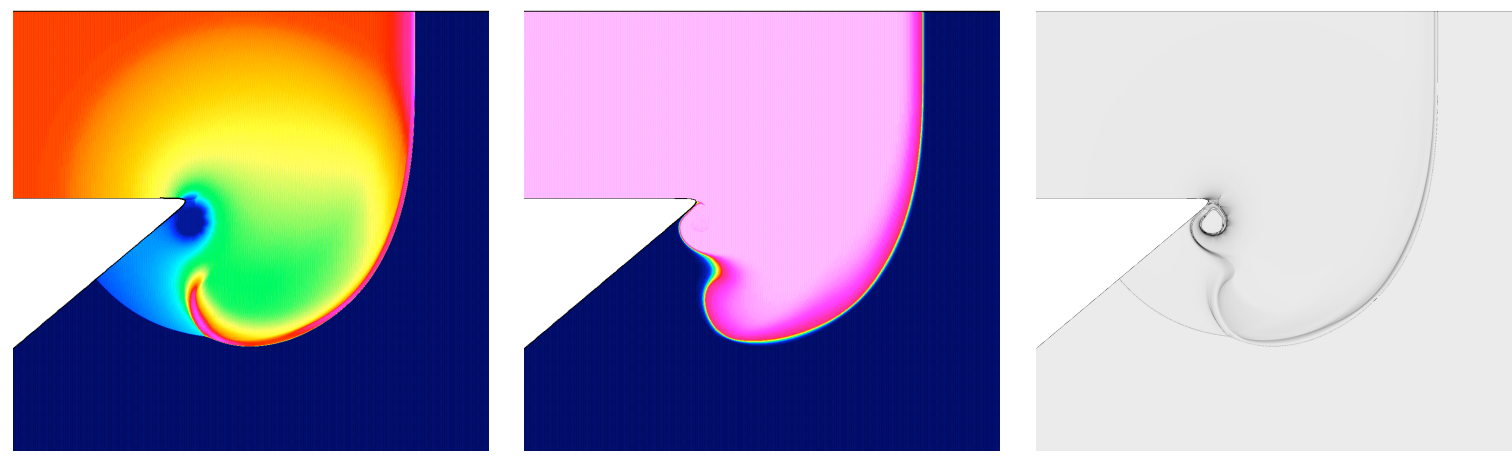

(b) $t=2.2$
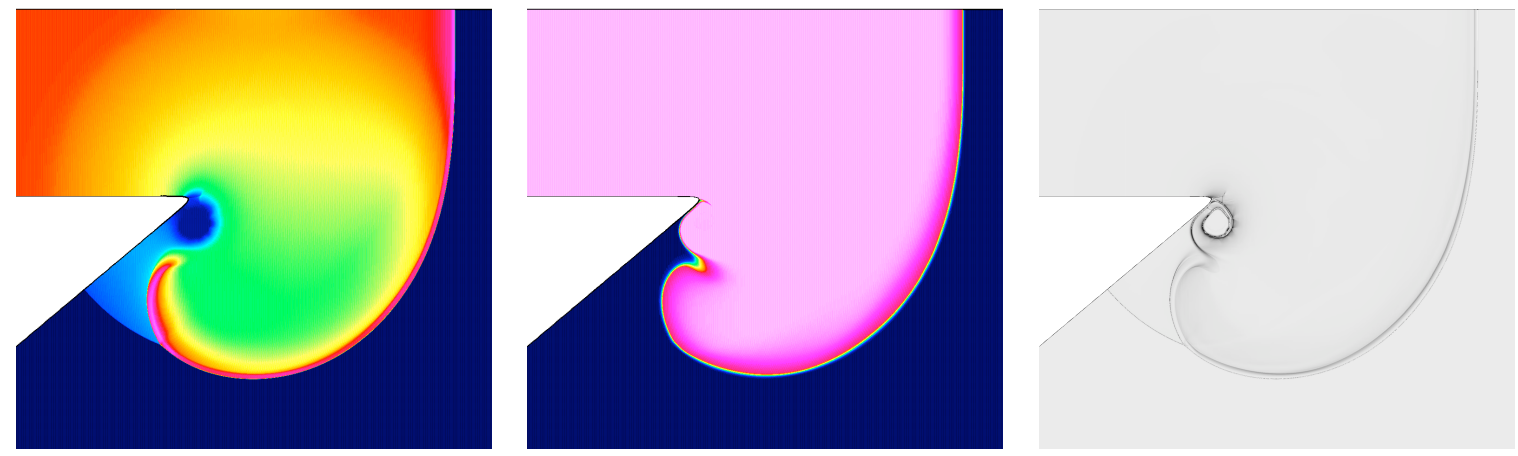

(c) $t=2.4$

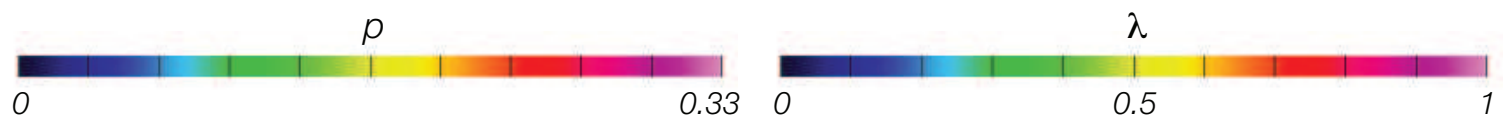

Figure 24: Contours of $p$ (left), $\lambda$ (middle) and numerically-generated schlieren (right) at at $t=2.0,2.2$ and 2.4 . 

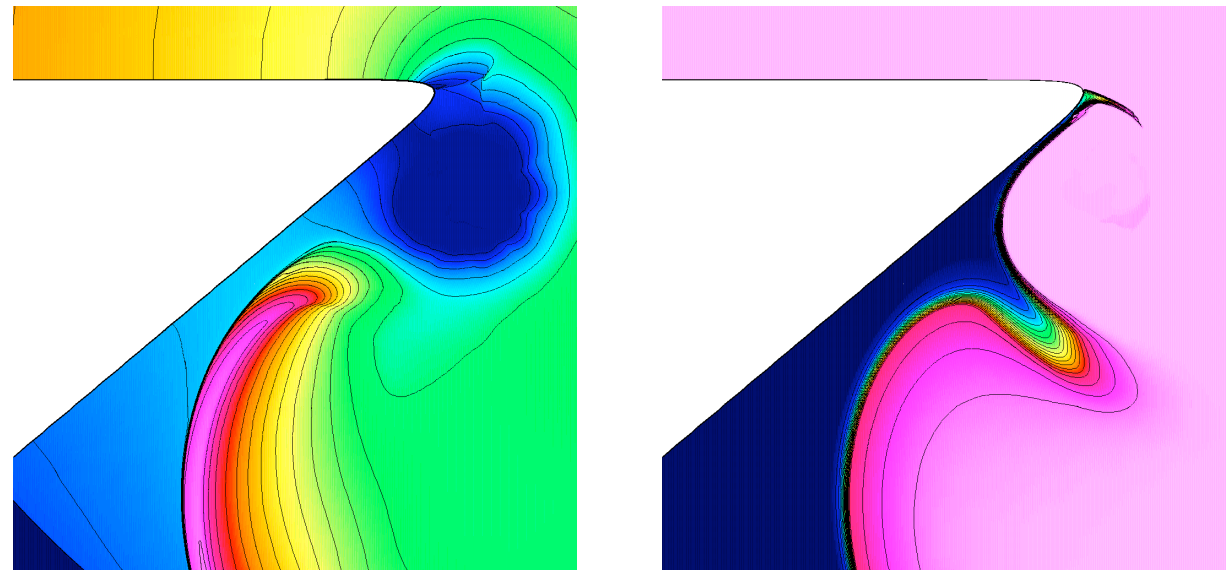

(a) $t=3.9$
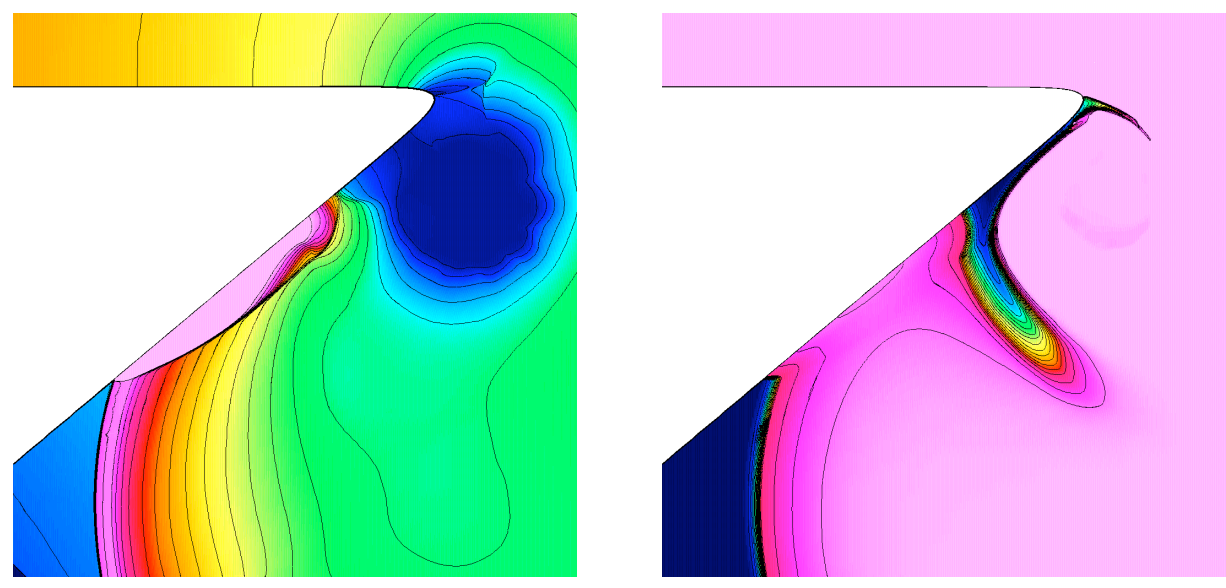

(b) $t=4.0$

Figure 25: Contours of pressure (left) and $\lambda$ (right) at $t=3.9$ and 4.0 .

the product region just created by the passage of the transverse detonation at a higher pressure. The pressure within this well is higher than that in the weakly-shocked dead pocket ahead of the transverse detonation. The fact that the contents of the well are reactive is indicated by the $\lambda$-gradient within the well.

At $t=2.6$, figure 25(b), the transverse wave has just reflected from the rigid wall. Figures $25(\mathrm{~b})$ and 26 (a), corresponding to $t=2.6$ and $t=2.7$ respectively, show that the area processed by the reflected shock has formed an oblong domain, increasing in size as the reflected shock travels back into the configuration, and with a profile that is blunt at the end closer to the corner and sharp at the end farther from it. Behind the reflected shock the pressure contours in these figures show the shock strength to be nonuniform, with the shock being stronger at the sharp end and weaker at the blunt end. The bulk of the reflected shock travels into material that is already fully reacted, having been processed by the forward-moving detonation. However, the blunt, low-pressure end of the reflected shock encounters the not-yet-fully-reacted material in the aforementioned well, figures 25(b) and 26(a). While the reflected shock hastens the consumption of this material, it is important to note that it would have fully reacted anyway. This is clearly evident in the $\lambda$ profile of figure $26(\mathrm{~b})$, which shows that the portion of the well yet unprocessed by the reflected shock is nearly fully reacted.

As was done for the case of the $90^{\circ}$ degree corner, it is useful to examine the profiles of density and $\lambda$ along the wall for further clarification. Figure 27(a) shows these profiles from $t=0.8$ to $t=1.4$, as the 

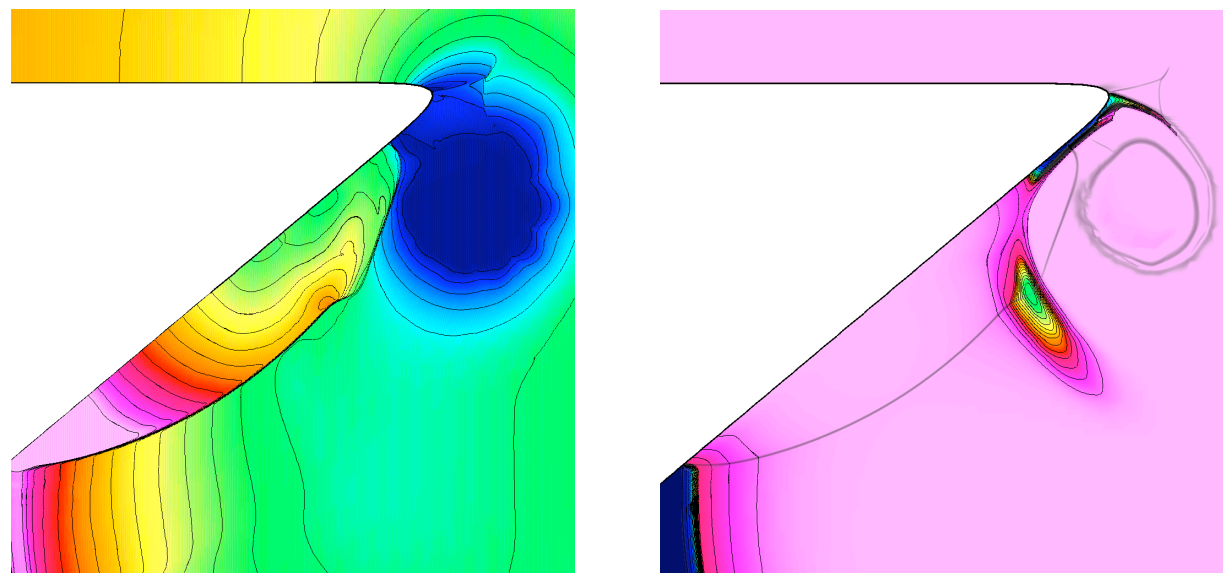

(a) $t=4.1$
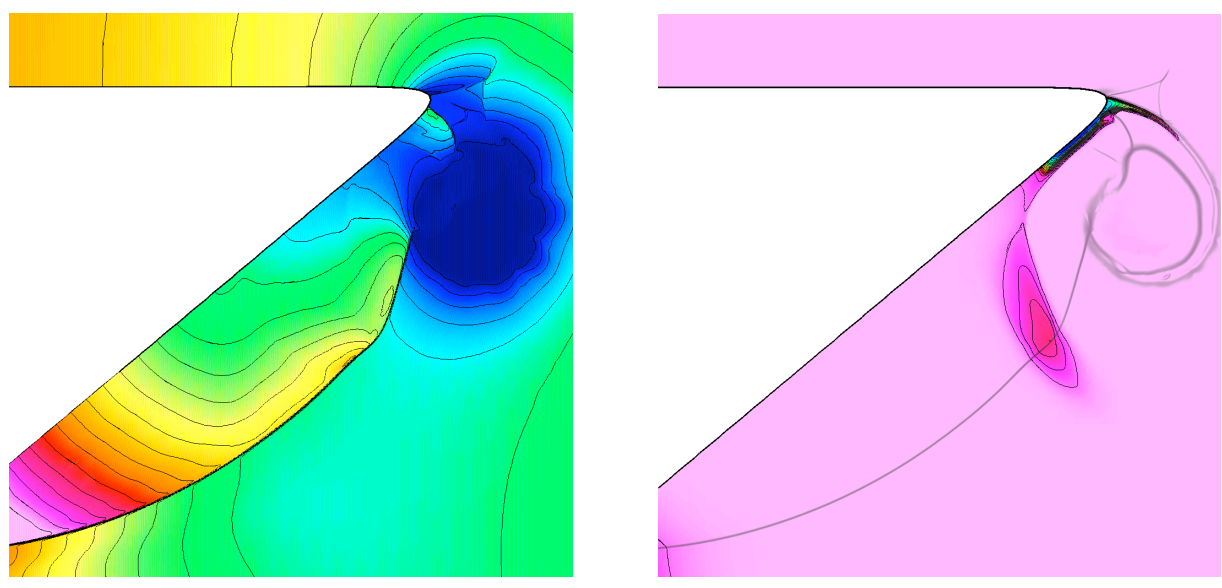

(b) $t=4.2$

Figure 26: Contours of pressure (left) and $\lambda$ (right) at $t=4.1$ and 4.2 .

detonation has just traversed the corner. As we saw for the $90^{\circ}$ degree corner, the plane, CJ structure at $t=0.8$ weakens upon diffraction and by $t=1.4$, a broad zone of unreacted material has accumulated behind the shock, separated from the fully reacted material behind it by a density jump. We note in particular that the density behind the lead shock has fallen below the threshold value 1.22 needed to initiate the ignition step, so that the reaction rate behind the shock has indeed fallen to zero. Figure 27(b) shows that the lack of reactivity continues till $t=2.4$, and indeed, till $t=2.5$ as is evident from figure 28 . At $t=2.6$ the situation in figure 28 changes dramatically, as the curved transverse wave has just hit the wall; recall figure 25(b). The broadening of the product region at the wall is clearly evident, as is the nonuniform pressure distribution at the wall. We also observe a narrow unreacted region near the corner, corresponding to entrainment of the reactant into the low-pressure corner vortex. 


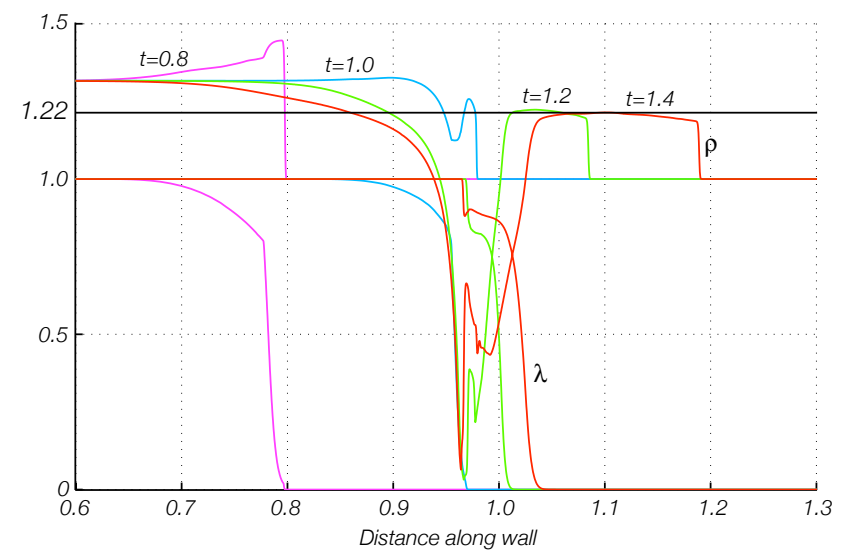

(a)

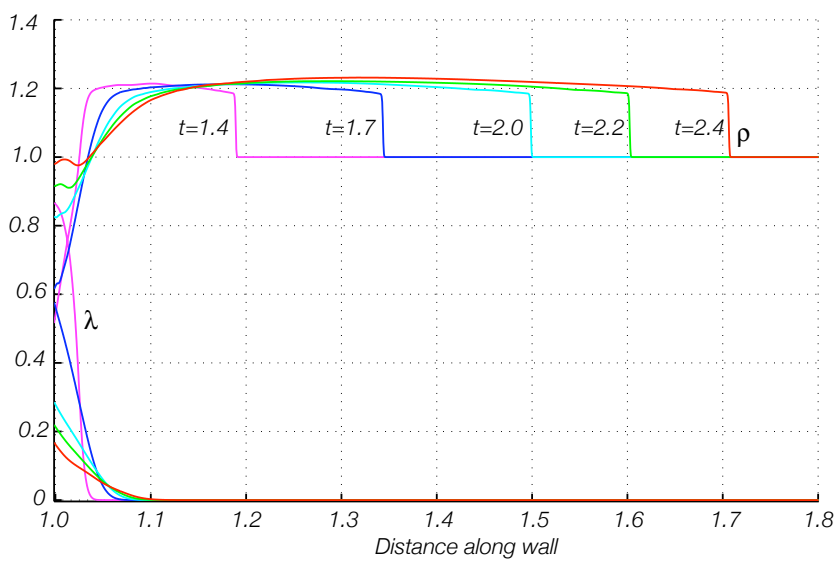

(b)

Figure 27: Profiles of reaction progress and density along the wall: (a) $t=0.8$ to 1.4 and (b) $t=1.4$ to 2.4 .

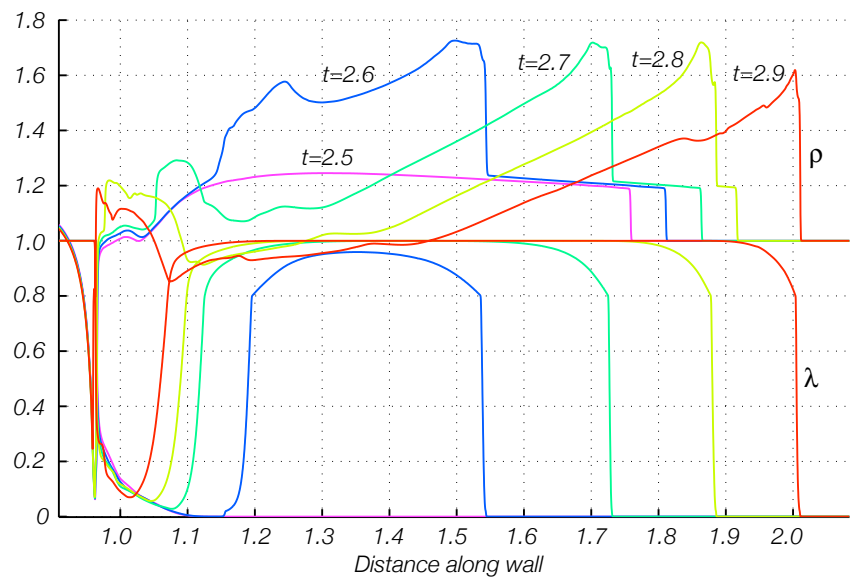

Figure 28: Profiles of reaction progress and density along the wall for $t=2.5$ to 2.9 . 


\section{Axisymmetric Diffraction Around a $90^{\circ}$ Corner}

We now consider a special axisymmetric configuration, the so-called hockey-puck geometry, also considered by Tarver [11], that combines spherical divergence with right-angled corner turning. The sample, a radial cross-section of which is shown in figure 29, consists of a solid right-circular cylinder from which a smaller cylinder has been removed to form a coaxial, hollow cylindrical core. A spherical detonation wave, pointinitiated at the center of the flat, circular surface of the core, encounters a right-angled corner when it evolves to a radius equal to the radius of the core. It is of interest to determine whether diffraction at this corner, now aided by the additional effect of divergence, will lead to the creation of sustained dead zones.

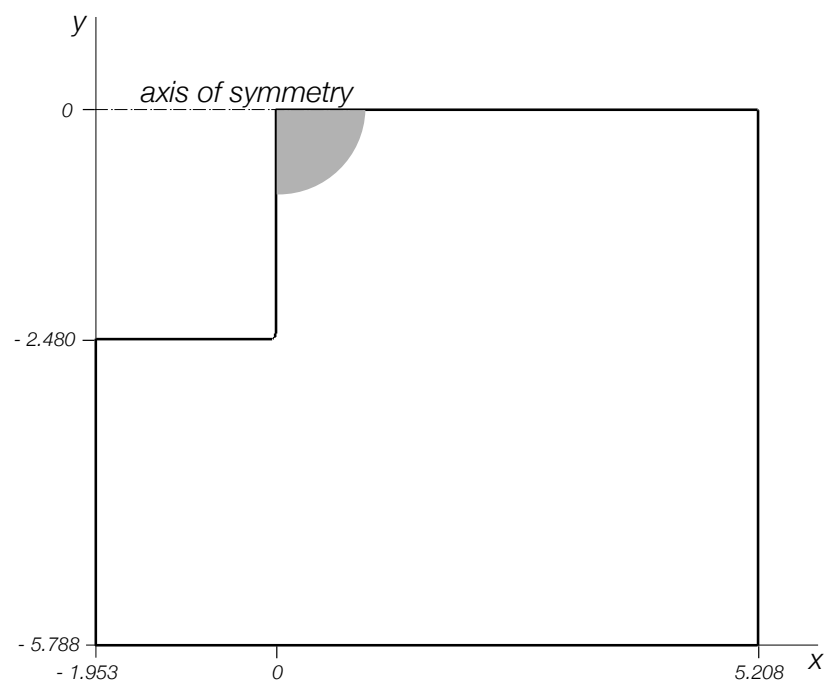

Figure 29: Computational configuration for diffraction around a $90^{\circ}$ corner in the axisymmetric geometry.

The computation begins at $t=0$, with an initial condition corresponding to a high-pressure, ambient density, parcel of product ( $\lambda=1, p=0.23$ and $v=1$ ) assumed to occupy a hemisphere of radius unity centered at the flat end of the core; see figure 29. The resulting spherically symmetric Riemann problem creates a detonation that is well-established by approximately $t=1.9$ when it reaches the corner. Diffraction and the subsequent evolution of the wave are displayed in the pressure, product and schlieren plots of figures $30-31$, that cover the period $t=1.9$ to $t=4.1$. In view of the cases already discussed, we can now afford to be brief. We observe that subsequent to corner turning the events near the corner consist of (i) separation of the reaction zone from the lead shock, (ii) formation of a transverse detonation, (iii) formation of a small pocket of reacting material between larger regions of complete consumption, (iv) reflection of the transverse wave from the wall, and (v) near-complete consumption of the reacting pocket. We note, in particular, the absence of sustained dead zones.

It is useful to consider the aftermath of wave reflection in additional detail to highlight the differences between the present case and the case of the $140^{\circ}$ corner. We begin with the exploded views in figure 32 (a), showing contours of $p$ and $\lambda$ at $t=3.9$. We note the weak primary shock that has apparently not been able to switch the reaction on, the main detonation advancing to the left, and the transverse detonation approaching the wall. We also see the sharp interface between reactant and product at the wall, as well as the narrow well of reacting material; these features are entirely analogous to what was seen earlier for the 140 degree corner.

In figure 32(b), corresponding to $t=4.0$, the transverse wave has just reached the wall. It is important to note that the material within the well has undergone substantial reaction, and that the transverse wave has essentially pinched the well off from its surroundings. At $t=4.1$, figure 33(a), the transverse wave has reflected off the wall. The collision is more head on, and the reflection rather more symmetric than was observed in figure $25(\mathrm{~b})$ for the $140^{\circ}$ corner. The well continues to react (see figure 33(b) corresponding to $t=4.2$ ) and the material within it is well on its way to complete reaction by $t=4.3$, see figure 34 . Unlike 

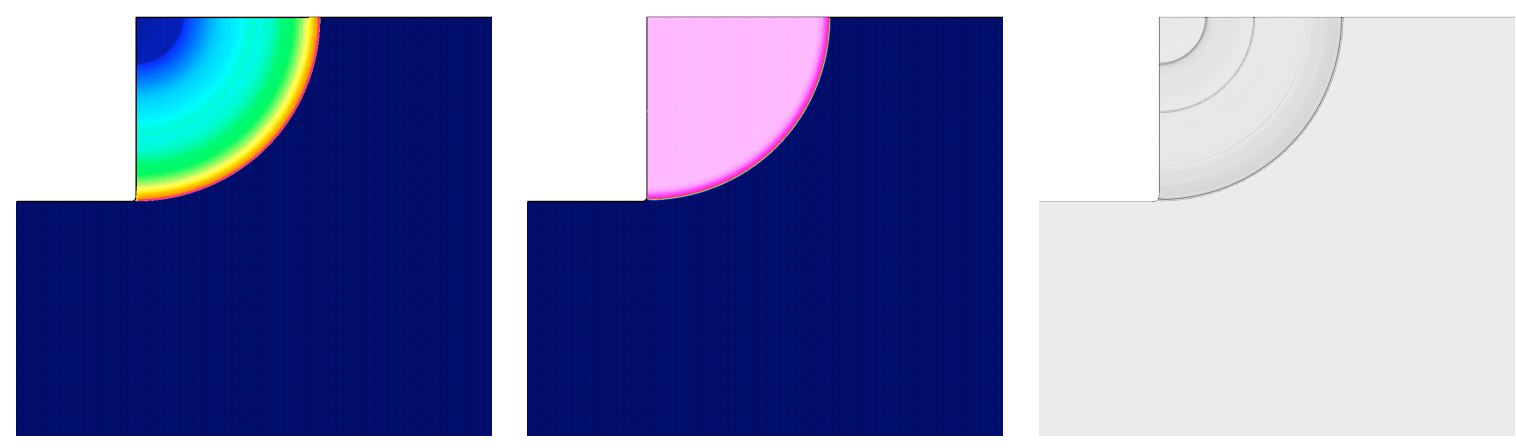

(a) $t=1.9$
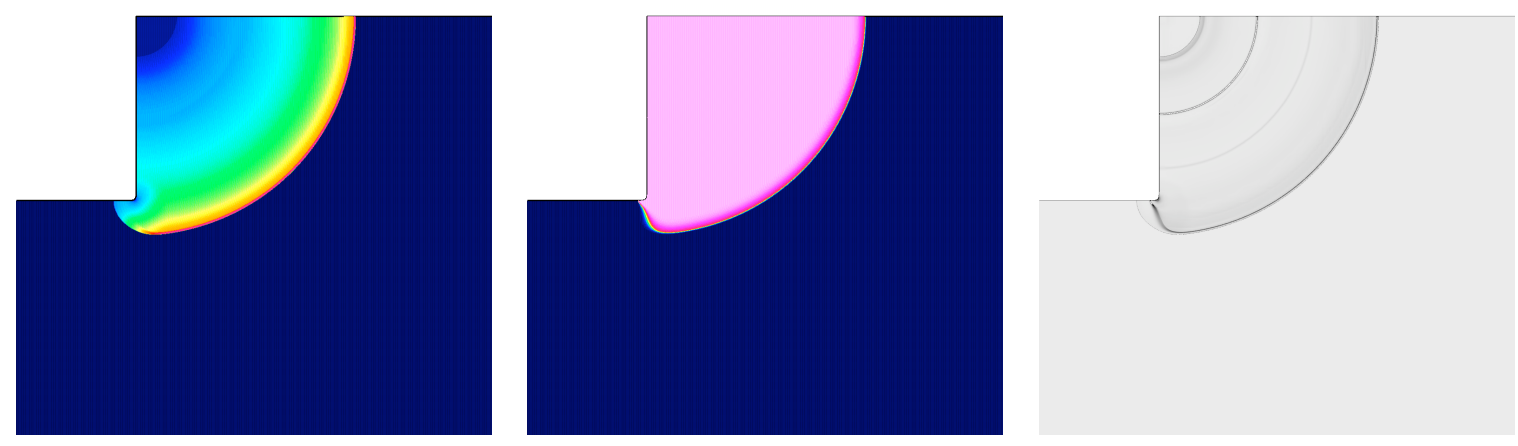

(b) $t=2.4$
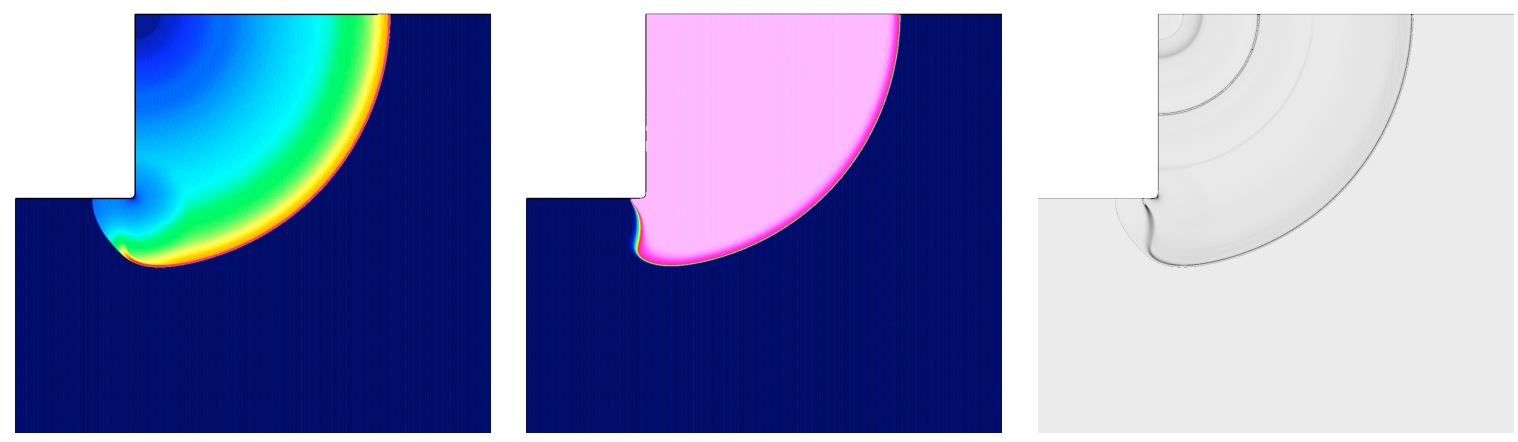

(c) $t=2.9$
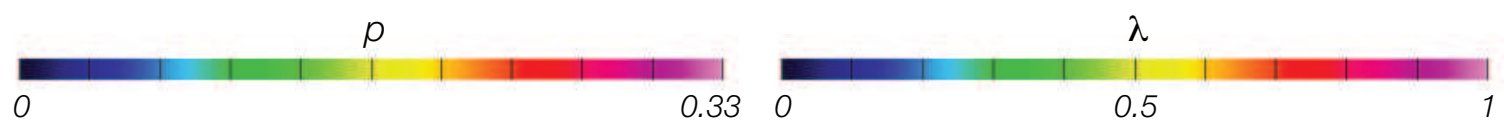

Figure 30: Contours of $p$ (left), $\lambda$ (middle) and numerically-generated Schlieren (right) at $t=1.9,2.4$ and 2.9 . 

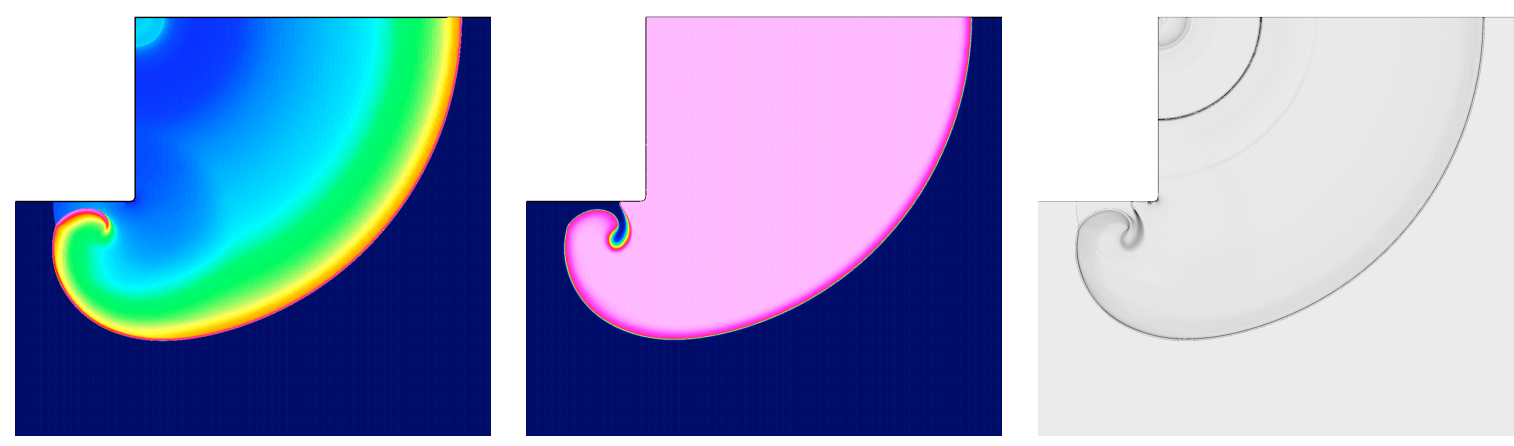

(a) $t=3.9$
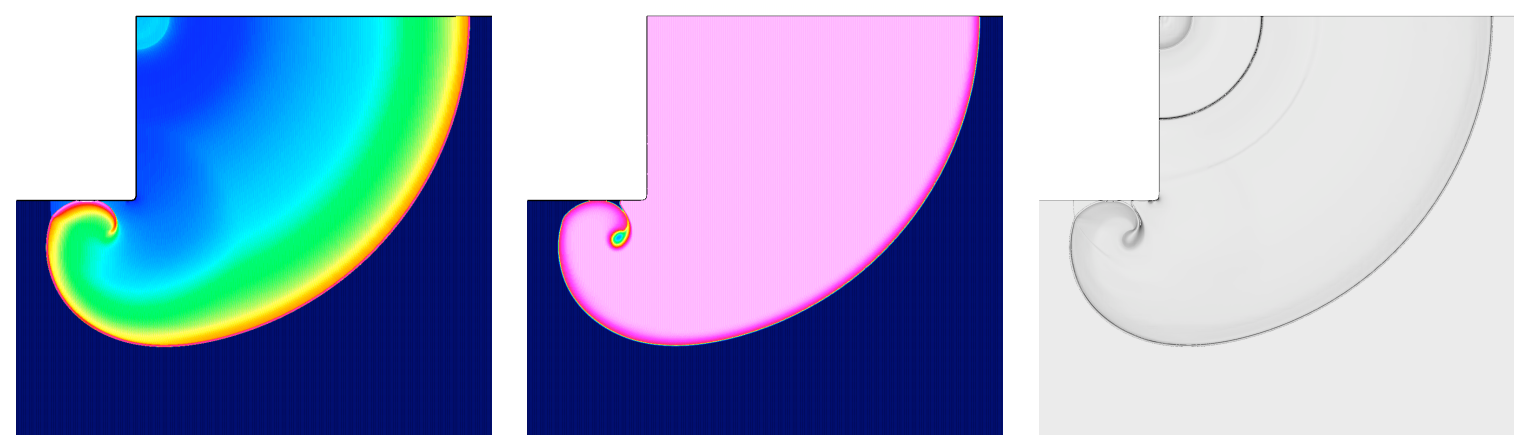

(b) $t=4.0$
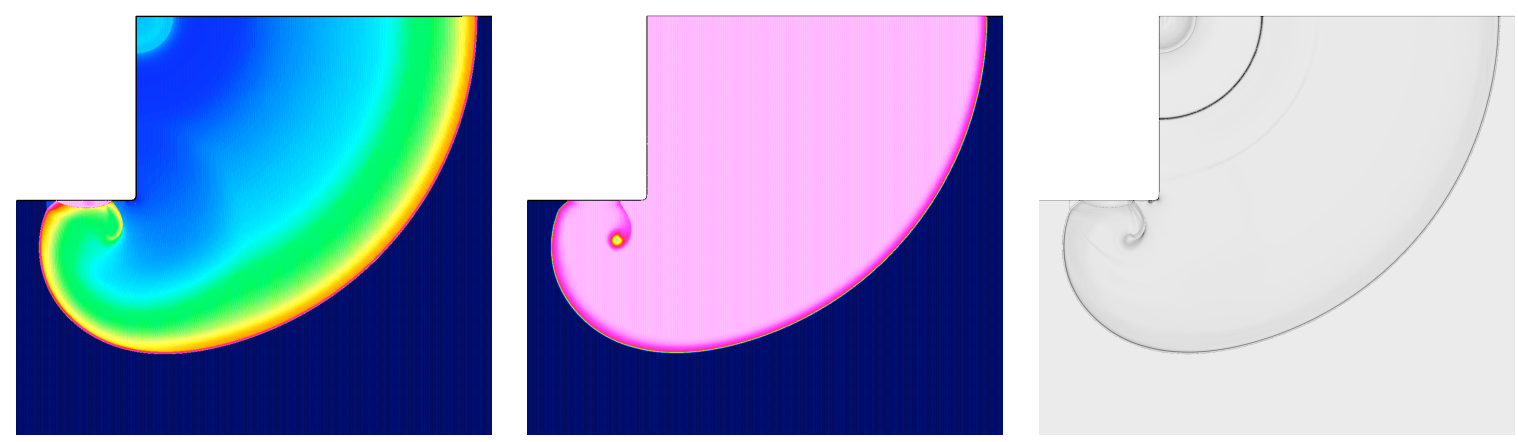

(c) $t=4.1$

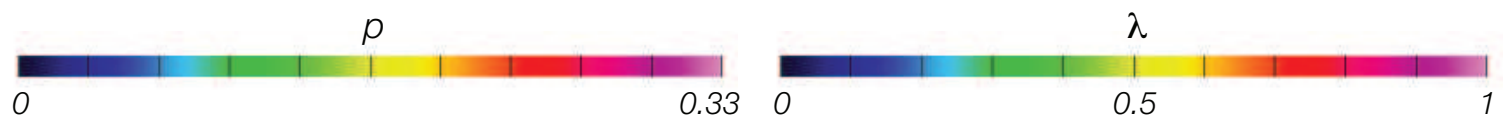

Figure 31: Contours of $p$ (left), $\lambda$ (middle) and numerically-generated Schlieren (right) at $t=3.9,4.0$ and 4.1 . 

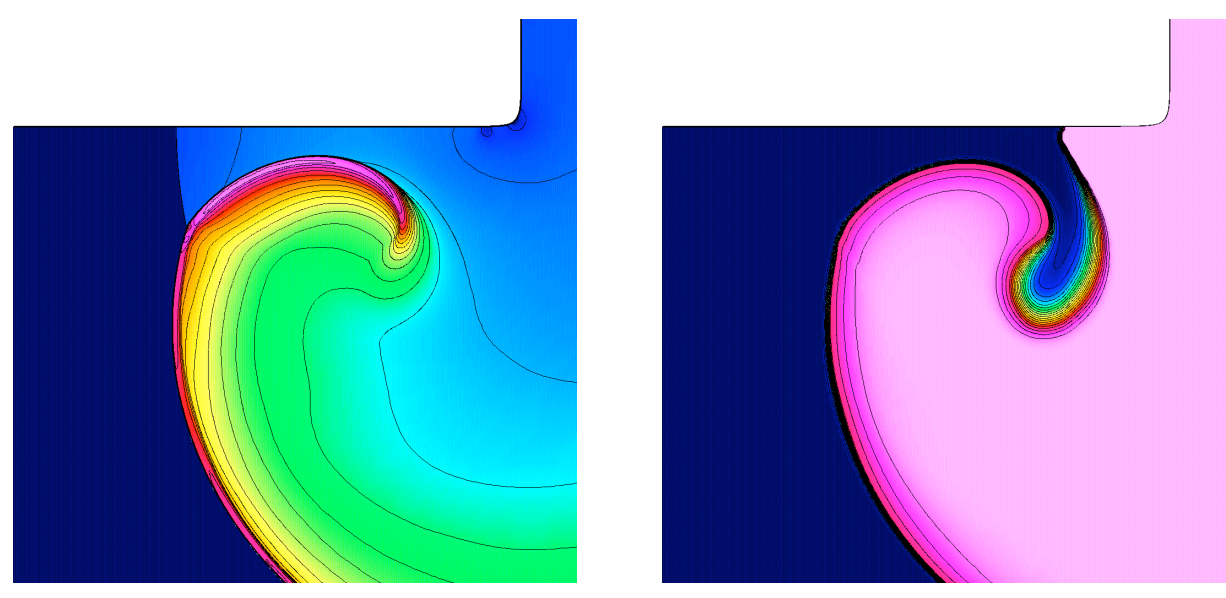

(a) $t=3.9$

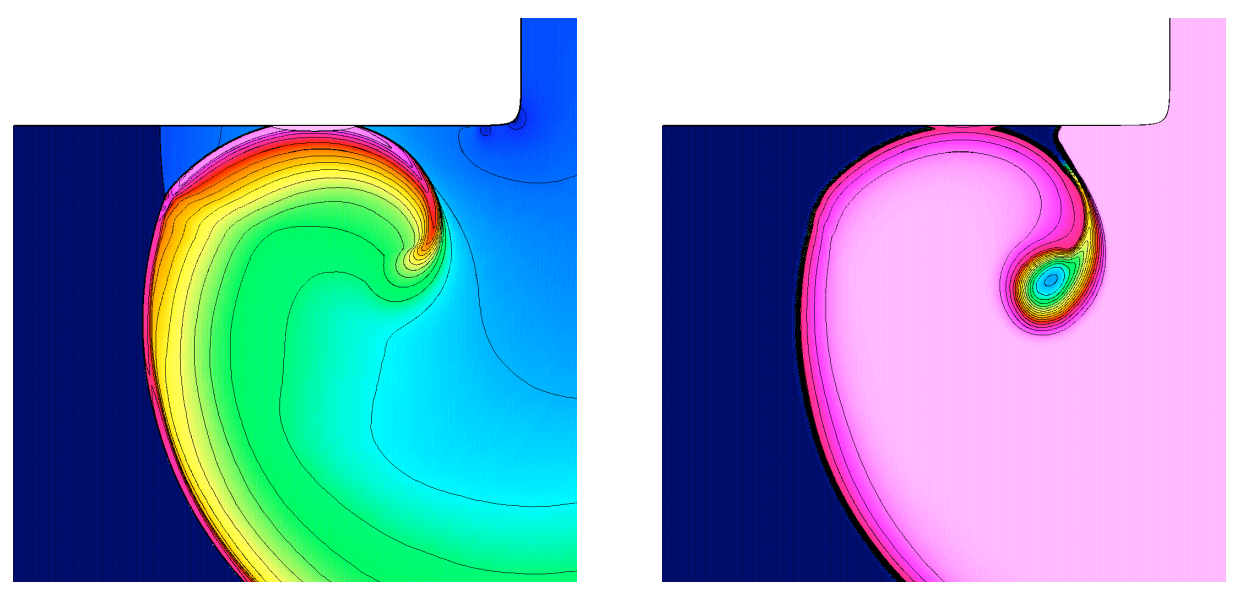

(b) $t=4.0$

Figure 32: Contours of pressure (left) and $\lambda$ (right) at $t=3.9$ and 4.0 .

the 140 degree case, the reflected shock plays no role in the complete consumption of the well.

We conclude from these observations that in none of the three cases considered is it possible for sustained dead zones to exist. According to the above discussion, this remains true even if the wall were nonreflecting or compliant.

\section{Conclusions}

In this paper we have subjected the ignition-and-growth model for heterogeneous explosives to a detailed study. The model was proposed by Lee and Tarver [5] more than twenty five years ago and has since been refined, and extensively applied to a large variety of explosives and configurations, by Tarver and colleagues. It has been demonstrated that the model supplies a framework within which a broad class of experiments can be simulated, provided one is willing to perform some tuning of the model parameters. This study is meant to be a substantial step towards establishing the solution set of the model, and identifying the phenomenology contained within it. Such an exercise had not been previously attempted, but is necessary if the strengths and weaknesses of the model are to be catalogued and understood. Only then can an intelligent attempt be made to modify and extend it, to cover situations for which the current version is inadequate. 


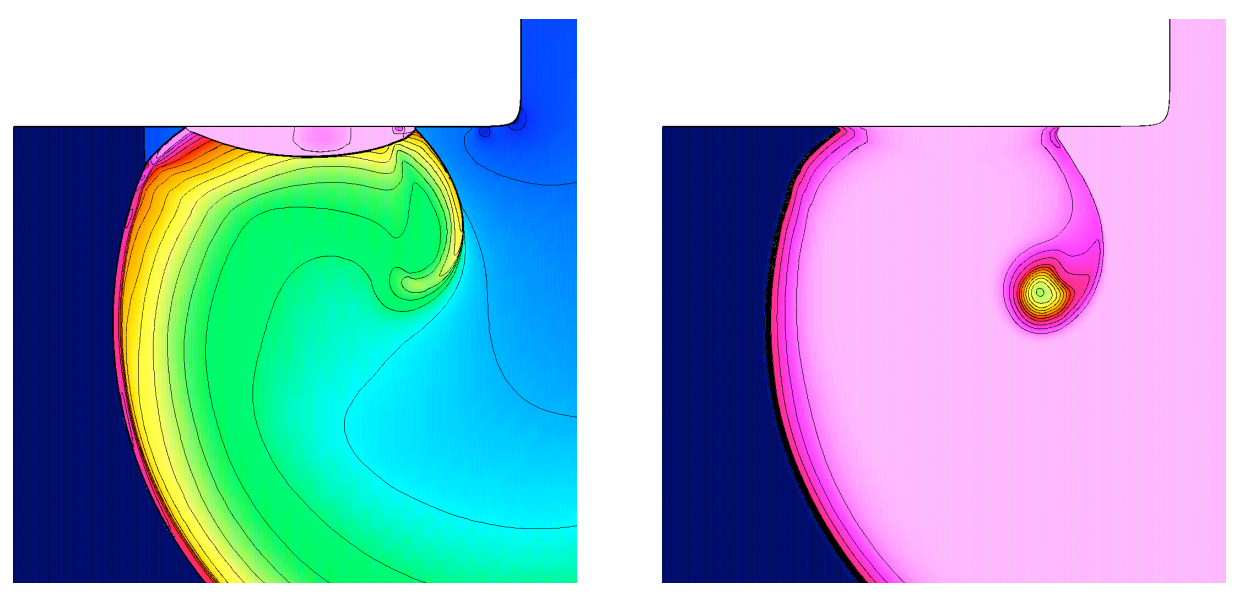

(a) $t=4.1$

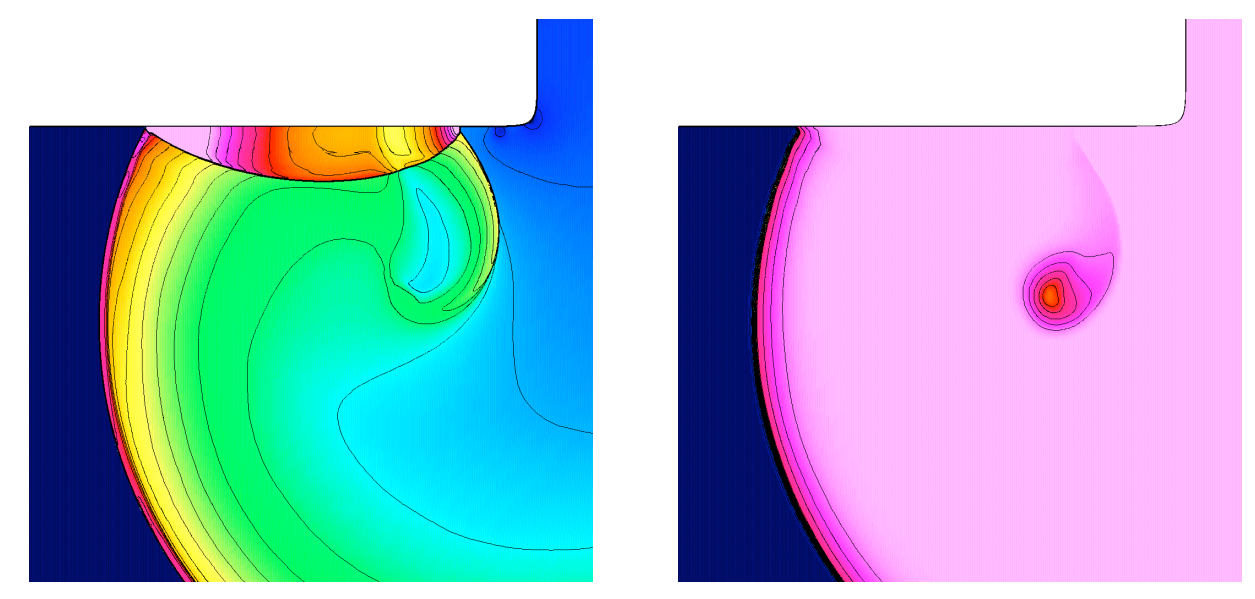

(b) $t=4.2$

Figure 33: Contours of pressure (left) and $\lambda$ (right) at $t=4.1$ and 4.2 .
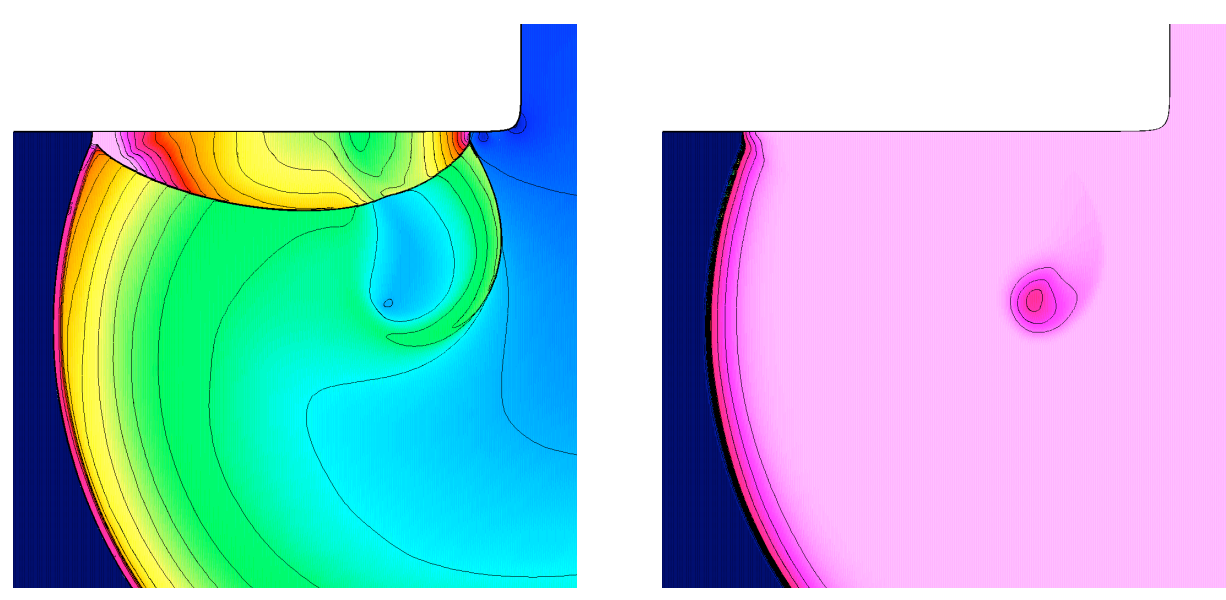

Figure 34: Contours of pressure (left) and $\lambda$ (right) at $t=4.3$. 
The study has concentrated, in this first instance, on rigidly confined explosives, and the model has been exercised on a set of configurations of practical relevance. A specific set of parameters proposed for detonation propagation in the explosive LX-17 is adopted. Steady Chapman-Jouguet structures are determined analytically, and these solutions are used as test beds for the numerical setup. Computational accuracy has been a prime concern, and an adaptive refinement strategy is employed to obtain well-resolved solutions with economy. Results are presented for shock-initiation of detonation in a planar, 1-D geometry. Two different scenarios are identified depending upon the strength of the applied stimulus. When the initiating shock is weak, detonation is produced by an explosion that occurs some distance behind the lead shock. The secondary shock so generated overtakes the lead shock and a CJ wave is established in due course. When the initiating shock is strong, it is the lead shock itself that is strengthened and accelerated by the reaction behind it, and the shock-reaction complex transitions into a detonation.

We have focused particular attention on detonation diffraction and possible detonation failure. This was motivated partially by a controversy in the recent literature about whether ignition-and-growth is capable of exhibiting sustained failure and dead zones; a feature that is thought to have been observed in corner turning experiments. We consider $90^{\circ}$ corners in planar and axi-symmetric geometries and find that although local decoupling of the lead shock and the reaction zone is possible, it is only temporary. These dormant regions are subject to revival either on their own because they were weakly preconditioned by the lead shock and ultimately explode, or because they can be swept by detonations generated elsewhere in the domain. We did not find sustained dead zones even in the extreme case of turning round a $140^{\circ}$ corner.

Even though the study was restricted to rigid confinement, we present arguments to contend that compliant boundaries would not alter the basic picture significantly. In particular, we assert that the model must be extended to include the effect of desensitization by weak shocks if it is to replicate sustained failure and dead zones. Such an extension is under development.

\section{Acknowledgements}

The authors thank Craig Tarver for his helpful input on the work. This study was partially supported by the Los Alamos National Laboratory, the Lawrence Livermore National Laboratory and the National Science Foundation.

\section{References}

[1] C. L. Mader, C. A. Forest, Two-dimensional homogeneous and heterogeneous wave propagation, Tech. Rep. LA-6259, Los Alamos Scientific Laboratory (1976).

[2] C. L. Mader, Two-dimensional homogeneous and heterogeneous wave propagation, in: The Sixth Symposium (International) on Detonation, 1976, pp. 405-413.

[3] J. N. Johnson, P. K. Tang, C. A. Forest, Shock wave initiation of heterogeneous reactive solids, J. Applied Phys. 57 (1985) 4323.

[4] J. Starkenberg, Modeling detonation propagation and failure using explosive initiation models in a conventional hydrocode, in: The Twelfth Symposium (International) on Detonation, 2002, pp. 10011007.

[5] E. L. Lee, C. M. Tarver, Phenomenological model of shock initiation in heterogeneous explosives, Phys. Fluids 23 (1980) 2362-2372.

[6] C. M. Tarver, J. O. Hallquist, Modelling two-dimensional shock initiation and detonation wave phenomena in PBX 9404 and LX-17, in: The Seventh Symposium (International) on Detonation, 1981, pp. $488-497$.

[7] C. M. Tarver, J. O. Hallquist, L. M. Erickson, Modelling short-pulse duration shock initiation of solid explosives, in: The Eighth Symposium (International) on Detonation, 1985, pp. 951-960. 
[8] C. M. Tarver, J. W. Kury, R. D. Breithaupt, Detonation waves in triaminonitrobenzene, J. Applied Phys. 82 (1997) 3771-3782.

[9] J. W. Kury, R. D. Breithaupt, C. M. Tarver, Detonation waves in trinitrotoluene, Shock Waves 9 (1999) $227-237$.

[10] C. M. Tarver, E. M. McGuire, Reactive flow modeling of the interaction of TATB detonation waves with inert materials, in: The Twelfth Symposium (International) on Detonation, 2002, pp. 641-649.

[11] C. M. Tarver, Ignition-and-growth modeling of LX-17 hockey puck experiments, Propellants, Explosives and Pyrotechnics 30 (2005) 109-117.

[12] P. C. Souers, R. Garza, P. Vitello, Ignition and growth and JWL++ detonation models in course zones, Propellants, Explosives and Pyrotechnics 27 (2002) 62-71.

[13] P. C. Souers, H. G. Andreski, C. F. C. III, R. Garza, R. Pastrone, D. Phillips, F. Roeske, P. Vitello, J. D. Molitoris, LX-17 corner turning, Propellants, Explosives and Pyrotechnics 29 (2004) 359-367.

[14] W. D. Henshaw, D. W. Schwendeman, An adaptive numerical scheme for high-speed reactive flow on overlapping grids, J. Comput. Phys. 191 (2003) 420-447.

[15] M. Arienti, J. E. Shepherd, A numerical study of detonation diffraction, J. Fluid Mech. 529 (2005) $117-146$.

[16] W. Fickett, W. C. Davis, Detonation, University of California Press, Berkeley, 1979.

[17] B. Dobratz, P. Crawford, LLNL explosives handbook, Tech. Rep. UCRL-52997, Lawrence Livermore National Laboratory (1985).

[18] M. J. Berger, J. Oliger, Adaptive mesh refinement for hyperbolic partial differential equations, J. Comput. Phys. 53 (1984) 484-512.

[19] P. L. Roe, Approximate Riemann solvers, parameter vectors, and difference schemes, J. Comput. Phys. 43 (1981) 357-372.

[20] P. Glaister, An approximate linearised Riemann solver for Euler equations for real gases, J. Comput. Phys. 74 (1988) 382-408.

[21] R. L. Gustavsen, S. A. Sheffield, R. R. Alcon, J. W. Forbes, C. M. Tarver, F. Garcia, Embedded electromagnetic gauge measurements and modeling of shock initiation in the tatb based explosives LX17 and PBX 9502, in: M. D. Furnish, N. N. Thadhani, Y. Horie (Eds.), Shock Compression of Condensed Matter, 2001, pp. 1019-1022.

This work was performed under the auspices of the U.S. Department of Energy by University of California, Lawrence Livermore National Laboratory under Contract W-7405-Eng-48. 